\title{
Kupfer-katalysierte Azid-Alkin-Cycloaddition zur Modifikation von Kohlenhydraten
}

\author{
Dissertation \\ zur Erlangung des Doktorgrades Dr. rer. nat. \\ der Fakultät für Forstwissenschaften und Waldökologie \\ der Georg-August-Universität Göttingen \\ vorgelegt von \\ Pascal Fuchs \\ geboren am 10.02.1991 in Wadern
}

Göttingen, Februar 2020 
1. Gutachter: Prof. Dr. Kai Zhang

2. Gutachter: Prof. Dr. Thomas Heinze

Tag der mündlichen Prüfung: 21.02.2020 


\section{Zusammenfassung}

Die Kupfer-katalysierte Azid-Alkin-Cycloaddition (CuAAC) ist der wohl bekannteste Vertreter der ClickChemie. Aufgrund ihrer einfachen Durchführung und hohen Ausbeute erfreut sie sich seit ihrer Entdeckung vor 17 Jahren wachsender Beliebtheit. Ihre Durchführung ist an Luft und in Wasser möglich, es gibt aber auch Katalysatorsysteme wie Kupfer(I)-iodid/ $N, N$-Diisopropylethylamin (DIPEA), die die Durchführung unter Wasser- und Luftausschluss erlauben.

In der Kohlenhydratchemie, insbesondere in der Cellulosechemie, ist die Anwendung dieses Katalysatorsystems weniger untersucht. Ein Ziel dieser Arbeit war die Synthese eines Cellulosederivates, welches mittels CUAAC mit Cul/DIPEA als Katalysatorsystem in wasserfreien organischen Lösungsmitteln umgesetzt werden kann. Im Anschluss sollte die erfolgreiche CuI/DIPEAkatalysierte Azid-Alkin-Cycloaddition an diesem Cellulosederivat demonstriert werden.

Dies wurde erreicht, indem eine neue Eintopfreaktion entwickelt wurde, die die effiziente Synthese von 6-Brom-6-desoxy-2,3-bis(trimethylsilyl)cellulose aus mikrokristalliner Cellulose (MCC) erlaubt. Bei der darauffolgenden nukleophilen Substitution des Broms gegen Azidgruppen wurde die organolösliche 6-Azid-6-desoxy-2,3-bis(trimethylsilyl)cellulose als Ausgangsmaterial für die Cul/DIPEAkatalysierte Azid-Alkin-Cycloaddition erhalten. Das so azidfunktionalisierte Cellulosederivat wurde in THF sowohl mit einem aliphatischen als auch mit einem aromatischen Alkin erfolgreich in der CuAAC unter Wasser- und Luftausschluss umgesetzt. So wurde demonstriert, dass Cellulose mittels CuAAC mit Cul/DIPEA als Katalysatorsystem in wasserfreien organischen Lösungsmitteln umgesetzt werden kann. Dies erweitert die Möglichkeiten, mit denen Cellulose mittels CuAAC modifiziert werden kann.

Ein weiteres Ziel meiner Arbeit war die Entwicklung einer Methode zur Herstellung linearer Polymere aus Monosaccharidderivaten mittels Cul/DIPEA-katalysierter Azid-Alkin-Cycloaddition. Dafür nötig war auch die Entwicklung eines geeigneten alkin- und azidfunktionalisierten Monosaccharidderivates. Bisher war die Polymerisation von bifunktionellen Monosaccharidderivaten zu linearen Polymeren nicht möglich gewesen, da die cyclische Oligomerisierung der Monomere als Hauptreaktion dominierte. Daher war die Minimierung cyclischer Nebenprodukte eine der Hauptherausforderungen.

Zunächst wurde das bifunktionelle Glucosederivat 2,3,6-Tris-O-benzyl-4-O-propargyl- $\beta$-Dglucopyranosylazid synthetisiert. Um die Cyclisierung dieses Derivates bei der CUAAC zu verhindern, wurde mit Startmolekülen gearbeitet, die entweder Azid- oder Alkingruppen enthalten. Ketten, die an diesen Startmolekülen wachsen, können mangels eines zweiten reaktiven Kettenendes nicht cyclisieren. Als erstes Startmolekül wurden Silica Nanopartikel (SiNPs) gewählt, deren Oberfläche mit einem zuvor synthetisierten Silanlinker azidfunktionalisiert wurde. Nach der CuAAC konnten die SiNPs inklusive der an ihnen gebundenen Polysaccharidanaloga durch Zentrifugation von den cyclischen 
Produkten in der Reaktionslösung abgetrennt und separat analysiert werden. Die Reaktionsbedingungen wurden optimiert, um eine möglichst geringe Ausbeute cyclischer Verbindungen und einen möglichst hohen Polymerisationsgrad auf der Oberfläche der SiNPs zu erhalten. Dies gelang durch die langsame und kontinuierliche Zugabe des Monomers über einen Zeitraum von 24 Stunden, was die effektive Monomerkonzentration während der Reaktion minimierte und so die Ausbeute linearer Verbindungen um 97 \% erhöhte. Jedoch lag die Ausbeute immer noch unter 20 \%. Im nächsten Schritt wurde diese Methode zur Synthese von Blockcopolymeren verwendet, indem das Monomer an Alkin-endfunktionalisertem Polyethylenglycol 2000 polymerisiert wurde. Hierbei wurden mit Ausbeuten über $90 \%$ ausschließlich lineare Polymere erhalten. Dies zeigt, dass das Katalysatorsystem Cul/DIPEA und die hier entwickelte Methode für die Synthese linearer Polysaccharidanaloga mit löslichen Startmolekülen hervorragend geeignet sind, jedoch nur eingeschränkt für die Synthese von Polysaccharidanaloga an Oberflächen empfohlen werden können. Die hier erhaltenen Ergebnisse legen nahe, dass das Katalysatorsystem Cul/DIPEA auch für die Polymerisation anderer (saccharidbasierter) Monomere verwendbar ist, unter der Voraussetzung, dass diese keine cyclischen Dimere zu bilden vermögen und die Polymerisation mit gut löslichen Ausgangsverbindungen durchgeführt wird. 


\section{Abstract}

The copper-catalyzed azide-alkyne cycloaddition (CuAAC) is one of the most prominent representatives of click chemistry. Due to its facile procedure and high yields it has been growing in popularity since its discovery 17 years ago. The reaction can be performed in the presence of water and air, but catalyst systems such as copper(I)-iodide/ $N, N$-diisopropylethylamine (DIPEA) also allow the reaction to be performed under inert atmosphere and moisture-free conditions.

In carbohydrate chemistry, especially in cellulose chemistry, the application of this catalyst system is less investigated. One goal of this thesis was the synthesis of a cellulose derivative which can be converted through CUAAC with Cul/DIPEA as catalyst system in organic solvents. Subsequently, the successful Cul/DIPEA-catalyzed azide-alkyne cycloaddition was to be demonstrated using this cellulose derivative.

This was accomplished by the development of a novel one-pot reaction which allows the efficient synthesis of 6-bromo-6-deoxy-2,3-tris(trimethylsilyl) cellulose, starting from microcrystalline cellulose (MCC). In the subsequent nucleophilic substitution of the bromide against azide groups the organosoluble 6-azido-6-deoxy-2,3-tris(trimethylsilyl) cellulose was obtained as starting material for the Cul/DIPEA-catalyzed azide-alkyne cycloaddition. The azide-functionalized cellulose derivative was dissolved in THF and successfully reacted via CuAAC with an aliphatic and an aromatic alkyne under moisture-free and oxygen-free conditions. It was thus demonstrated that cellulose can be converted with Cul/DIPEA as catalyst system in anhydrous organic solvents by means of CuAAC. This further expands the scope of possible click reactions on cellulose.

Another goal of my work was the development of a method for the preparation of linear polymers from monosaccharide derivatives by Cul/DIPEA-catalyzed azide-alkyne cycloaddition. This also required the development of a suitable alkyne- and azide-functionalized monosaccharide derivative. Until now, the polymerization of bifunctional monosaccharide derivatives into linear polymers was not possible because the cyclic oligomerization of the monomers dominated as the main reaction. Therefore, minimizing cyclic by-products has been a major challenge.

First, the bifunctional glucose derivative 2,3,6-tris-O-benzyl-4-O-propargyl- $\beta$-D-glucopyranosylazide was synthesized. In order to prevent the cyclization of this derivative during the CUAAC, starter molecules were used which contained either azide or alkyne groups. Chains growing from the starter molecules cannot cyclize due to the lack of a second reactive chain end. As a first starter molecule silica nanoparticles (SiNPs) were chosen. Azide-functionality was introduced to their surface with a previously synthesized azide-bearing silane linker. After the CUAAC was finished, the SiNPs, including the polysaccharide analogues bound to them, could be separated from the cyclic products in the 
reaction solution by centrifugation and were analyzed separately. The reaction conditions were optimized in order to obtain the lowest possible yield of cyclic compounds and the highest possible degree of polymerization on the surface of the SiNPs. This was achieved by slow and continuous addition of the monomer over a period of 24 hours, which minimized the effective monomer concentration during the reaction, increasing the yield of linear compounds by $97 \%$. However, the yield was still below $20 \%$. In the next step, this method was used to synthesize block copolymers by polymerizing the monomer on alkyne end-functionalized polyethylene glycol 2000 . Here, with yields above $90 \%$, only linear polymers were obtained. This shows that the Cul/DIPEA catalyst system and the method developed here are suitable for the synthesis of linear polysaccharide analogues with soluble starter molecules but can only be recommended to a limited extent for the synthesis of polysaccharide analogues on surfaces. The results obtained here suggest that the Cul/DIPEA catalyst system is also applicable for the polymerization of other (saccharide-based) monomers, provided that they cannot form cyclic dimers and the polymerization is carried out with a readily soluble starter molecule. 


\section{Inhaltsverzeichnis}

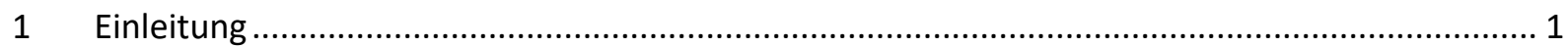

1.1 Kupfer-katalysierte Azid-Alkin-Cycloaddition: Paradebeispiel der Click-Chemie .................. 1

$1.2 \quad$ Katalysatoren für die Kupfer-katalysierte Azid-Alkin-Cycloaddition ................................. 2

1.3 Funktionalisierung von Cellulose für die CuAAC .......................................................... 3

1.4 Kupfer-katalysierte Azid-Alkin-Cycloaddition an Monosacchariden .................................. 7

1.4.1 Funktionalisierung der Monomere für die CuAAC ...................................................... 7

1.4.2 Polymerisation von Monosacchariden mittels CuAAC ................................................ 10

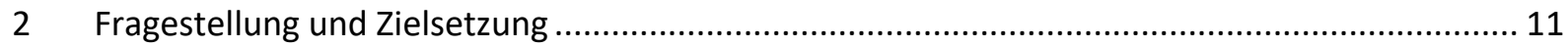

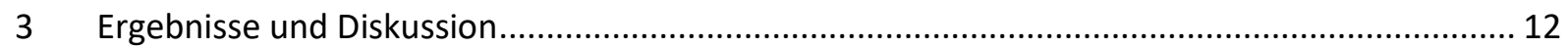

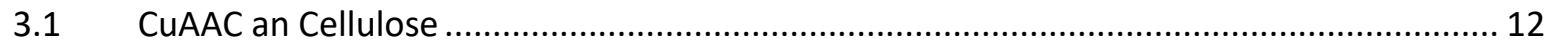

3.1.1 Synthese von 6-Azid-6-desoxy-2,3-bis(trimethylsilyl)cellulose ..................................... 12

3.1.2 CuAAC an 6-Azid-6-desoxy-2,3-bis(trimethylsilyl)cellulose .......................................... 15

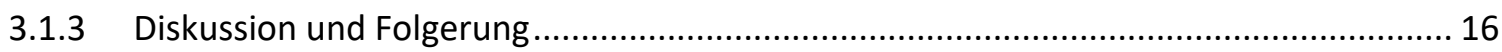

3.2 CUAAC-Polymerisation eines Monosaccharidderivates ................................................... 17

3.2.1 Synthese eines geeigneten monosaccharidbasierten Monomers ................................. 18

3.2.2 CuAAC-Oligomerisation an der Oberfläche von Silica-Nanopartikeln ............................ 19

3.2.3 CuAAC-Polymerisation an endfunktionalisiertem Polyethylenglycol............................. 22

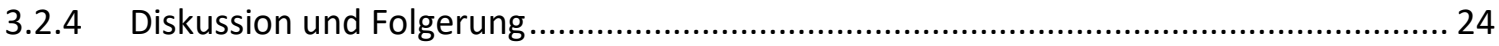

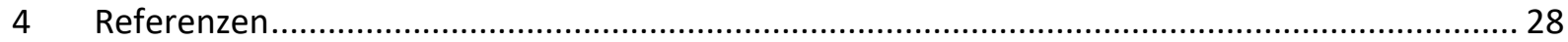

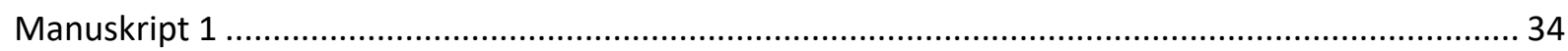

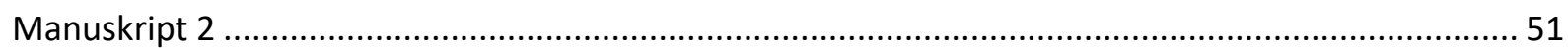

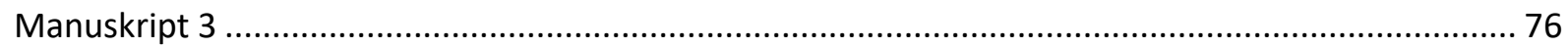




\section{Abkürzungsverzeichnis}

AGU

BET

CUAAC

$Ð$

DIPEA

DMAC

DMF

DMSO

DP

$D P_{n}$

$\mathrm{DP}_{\mathrm{w}}$

DS

GPC

HMDS

HPLC

MALDI

MCC

$M_{n}$

$\mathrm{M}_{\mathrm{w}}$

NBS

NMR

PLA

PS

Anhydroglucose-Einheit

Analyseverfahren zur Bestimmung von Oberflächen mittels Gasadsorption, benannt nach Brunauer, Emmett und Teller

Kupfer-katalysierte Azid-Alkin-Cycloaddition

Dispersität, $M_{w} / M_{n}$

$\mathrm{N}, \mathrm{N}$-Diisopropylethylamin

$\mathrm{N}, \mathrm{N}$-Dimethylacetamid

$N, N$-Dimethylformamid

Dimethylsulfoxid

Polymerisationsgrad

Zahlengemittelter Polymerisationsgrad

Gewichtsgemittelter Polymerisationsgrad

Substitutionsgrad

Gel-Permeations-Chromatographie

Hexamethylendisilazan

Hochleistungsflüssigkeitschromatographie (engl. high performance liquid chromatography)

Matrix-Assistierte Laser-Desorption-lonisierung

Mikrokristalline Cellulose

Zahlenmittel der Molmasse

Gewichtsmittel der Molmasse

$N$-Bromsuccinimid

Kernspinresonanz (engl. nuclear magnetic resonance)

Polylactid

Polystyrol 
SEM Rasterelektronenmikroskop (engl. scanning electron microscope)

SiNPs Silica Nanopartikel

SiNPs- $\mathrm{N}_{3} \quad$ Azidfunktionalisierte Silica Nanopartikel

SiNPs-P $20 \quad$ Silica Nanopartikel nach Oligomerisation mit 20 äq. Monomer.

SiNPs- $\mathrm{P}_{77} \quad$ Silica Nanopartikel nach Oligomerisation mit 7x11 äq. Monomer (stündliche Zugabe)

SiNPs-P $\quad$ Silica Nanopartikel nach Oligomerisation mit 80 äq. Monomer.

SiNPs-P ${ }_{80} \quad$ Silica Nanopartikel nach Oligomerisation mit 80 äq. Monomer (kontinuierliche Zugabe)

THF Tetrahydrofuran

TMS Trimethylsilyl-Gruppe

Ts $\quad p$-Toluensulfonyl-Gruppe 



\section{Einleitung}

\subsection{Kupfer-katalysierte Azid-Alkin-Cycloaddition: Paradebeispiel der Click-Chemie}

Die Click-Chemie wurde erstmals von KolB et al. im Jahr 2001 erwähnt. ${ }^{1}$ Damit werden Reaktionen beschrieben, mit denen kleine Molekülbausteine verlässlich im kleinen oder großen Maßstab miteinander verbunden werden können. Hierdurch soll die Synthese größerer Moleküle aus Fragmenten vereinfacht werden. Anwendung kann dies unter anderem bei der Entwicklung und Synthese von Molekülen mit biologischer Aktivität finden.

Um eine Reaktion als Click-Reaktion bezeichnen zu dürfen, muss diese modular und umfangreich anwendbar sein und gleichzeitig mit sehr hohen Ausbeuten ablaufen. Anfallende Nebenprodukte sollten durch nicht-chromatographische Methoden einfach abtrennbar sein. Des Weiteren sollten die Reaktionen stereospezifisch ablaufen und die Reaktionsbedingungen möglichst simpel sein. Im Idealfall sind diese Reaktionen nicht wasser- und sauerstoffempfindlich. Außerdem sollten die Ausgangsmaterialien leicht verfügbar sein, kein Lösungsmittel, Wasser oder ein einfach zu entfernendes Lösungsmittel benötigt werden und das Produkt sollte einfach zu isolieren sein. ${ }^{1}$ Seit der ersten Erwähnung der Click-Chemie wurden mehr als 14300 Publikationen zu diesem Thema verfasst. ${ }^{2}$ Reaktionen, die diesen Anforderungen entsprechen, sind beispielsweise Cycloadditionen ungesättigter Verbindungen, insbesondere 1,3-dipolare Cycloadditionen oder auch Diels-Alder-artige Reaktionen. Die Chemie der 1,3-dipolaren Cycloadditionen wurde schon in den 1960er Jahren von R. HUISGEN umfangreich untersucht und beschrieben. ${ }^{3,4}$ Darunter befindet sich auch die Cycloaddition eines Azids und eines Alkins, die unter Bildung eines Triazolrings abläuft. 2002 haben M. MELDAL und B. SHARPLESS zeitgleich berichtet, dass die Azid-Alkin-Cycloaddition durch Kupfer(I) katalysiert werden kann und damit schneller und regioselektiv zum 1,4-substituierten Triazol abläuft (Abbildung 1).5,6 Aufgrund dieses Durchbruches wurde die Kupfer-katalysierte Azid-Alkin-Cycloaddition (CuAAC) zum wohl bekanntesten Vertreter der Click-Reaktionen.

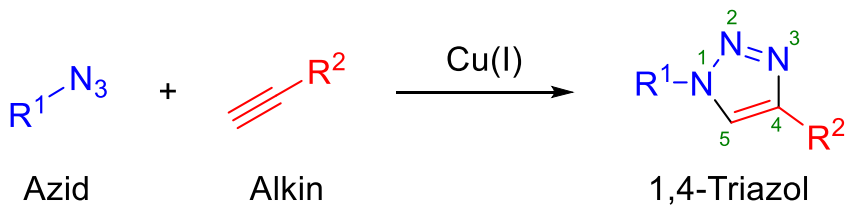

Abbildung 1: Allgemeines Reaktionsschema der Kupfer-katalysierten Azid-Alkin-Cycloaddition. 
Die thermodynamisch kontrollierte CuAAC zeichnet sich durch ihre hervorragende Kompatibilität mit vielen funktionellen Gruppen und vielen Lösungsmitteln einschließlich Wasser, einfache und milde Reaktionsbedingungen, sowie hohe pH-Wert-Toleranz aus. Dadurch wird die CuAAC zu einem wertvollen Werkzeug zur Synthese und Modifikation von komplexen organischen Grundgerüsten und biologisch relevanten Molekülen. Die Alkin- und Azidfunktionalitäten können einfach in ein Molekül eingebracht werden und gehen kaum Nebenreaktionen mit anderen funktionellen Gruppen und üblichen Reagenzien ein. Deshalb können diese funktionellen Gruppen unbeschadet durch viele Synthesestufen getragen werden. Dies erhöht weiter die Signifikanz der CuAAC und hat einen großen Anteil am Erfolg und der breiten Anwendbarkeit. ${ }^{6,7}$

\subsection{Katalysatoren für die Kupfer-katalysierte Azid-Alkin-Cycloaddition}

Bei der CuAAC ist eine Kupfer(I)-spezies katalytisch aktiv. Bestandteil vieler Untersuchungen war der Reaktionsmechanismus der CuAAC. ${ }^{8-10}$ Schließlich wurde ein Mechanismus vorgeschlagen, bei dem zwei Kupferatome an einem Katalysezyklus beteiligt sind (Abbildung 2$).{ }^{11}$

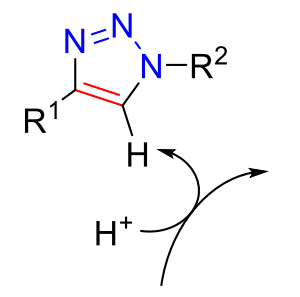

[Cu]
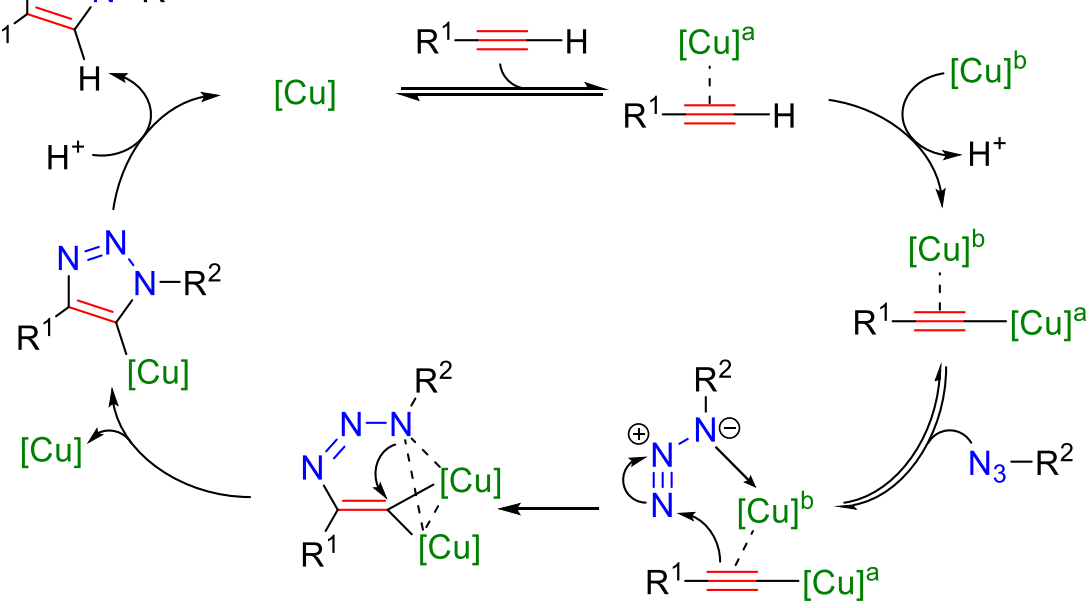

Abbildung 2: Vorgeschlagener Reaktionsmechanismus der CUAAC von WORREL, MALIK UND FOKIN. (Reproduziert aus ${ }^{11}$, Copyright genehmigt von The American Association for the Advancement of Science).

Eine der einfachsten und zuverlässigsten Quellen für das Kupfer in der CuAAC sind Kupfer(II)-salze. Beispielsweise kann Kupfer(II)-sulfat durch Natriumascorbat, welches im Überschuss zugegeben wird, reduziert werden. ${ }^{12}$ Hierbei werden viele funktionelle Gruppen, wie z.B. Amine, Alkohole oder Carboxylsäuren toleriert. Die Reaktion kann in wässrigen Lösungen durchgeführt werden oder in Gemischen von Wasser mit unterschiedlichen Alkoholen oder mit Dimethylsulfoxid (DMSO). ${ }^{13}$ Ein 
großer Vorteil der Reduktion von Kupfer(II)-salzen ist, dass diese Methode neben Wasser auch Sauerstoff toleriert. Gleichzeitig stellt dies aber auch einen limitierenden Faktor für diese Methode dar, da wasser- und sauerstoffempfindliche Substrate mit diesem Protokoll nicht umgesetzt werden können.

Neben der Reduktion eines Kupfer(II)-salzes zu Kupfer(I) besteht auch die Möglichkeit direkt ein Kupfer(I)-Salz zu verwenden. Diese Methode verlangt wasserfreie Lösungsmittel, da Kupfer(I) im Wässrigen zu Kupfer(0) und Kupfer(II) disproportioniert, sowie eine inerte Atmosphäre, um die Oxidation des Katalysators zu Kupfer(II) zu verhindern. Außerdem ist die Zugabe einer Stickstoff-Base wie Pyridin, N,N-Diisopropylethylamin (DIPEA) oder Triethylamin nötig, die gleichzeitig auch als Ligand für die Kupfer(I)-spezies dienen kann. ${ }^{13}$ Die Zugabe einer Amin-Base zur Deprotonierung des Alkins ist nur in aprotischen Lösungsmitteln nötig, da sich das entsprechende Kupfer(I)-acetylid in wässrigen Medien auch noch unter sauren Bedingungen bildet. ${ }^{14}$ DIPEA scheint als Base hervorragend geeignet zu sein, da dieses Amin zudem auch die CuAAC an Oberflächen wie beispielsweise der von Microtiterplatten oder Harzen ermöglicht. ${ }^{5,13,15}$ Gute Ergebnisse ließen sich auch in Toluol mit Kupfer(I)-iodid, -chlorid oder -bromid als Katalysator, Phosphorliganden wie beispielsweise Triphenylphosphin, und einer Base wie beispielsweise Kaliumacetat erzielen. ${ }^{13}$

Kupfer(I)-Salze eignen sich zudem als Katalysator für die CuAAC-Polymerisation in organischen Medien zur Bildung von Polytriazolen, die in wässrigen Medien agglomerieren würden. ${ }^{16}$ Gute Ergebnisse wurden bei dieser Anwendung mit (Triethylphosphit)kupfer(I)-iodid ([CulP(OEt) $\left.)_{3}\right]$ ) erzielt. Katalysatoren wie Tris(triphenylphosphin)kupfer(I)-bromid ([CuBr$\left.\left.\left(\mathrm{PPh}_{3}\right)_{3}\right]\right)$ sind hierfür weniger geeignet, da die Staudinger Reaktion zwischen Triphenylphosphin und Azidgruppen einen negativen Einfluss auf das erhaltene Molekulargewicht hat. ${ }^{17}$

\subsection{Funktionalisierung von Cellulose für die CuAAC}

Cellulose ist der Hauptbestandteil pflanzlicher Zellwände und damit das weltweit am häufigsten vorkommende Biopolymer. Durch ihre hohe jährliche Produktion als Biomasse gilt sie als beinahe unerschöpflich und stellt ein wertvolles Ausgangsmaterial für die Herstellung umweltfreundlicher und biokompatibler Produkte dar. ${ }^{18}$

Schon seit tausenden Jahren wird sie von Menschen verwendet. Die fast ausschließlich aus Cellulose bestehende Baumwolle diente schon im Alten Ägypten zur Herstellung von Kleidung. Holz wurde auch damals schon als Baumaterial und Energiequelle genutzt. Als ein Bestandteil des ägyptischen Papyrus nahm sie eine wichtige Position in der Entwicklung der menschlichen Kultur ein. ${ }^{19}$ 
Vor rund 160 Jahren wurde Cellulose erstmals als Ausgangsmaterial für die großtechnische chemische Synthese verwendet. Aus ihr wurde in einer Veresterung mit Salpetersäure Cellulosenitrat hergestellt, das versetzt mit dem Weichmacher Campher bis heute unter dem Markenamen Zelluloid Verwendung findet. Spätere Produkte wie Methyl-, Ethyl- oder Hydroxylalkylether der Cellulose, aber auch Celluloseacetat sowie viele weitere Cellulosederivate werden noch heute verwendet. Auch Celluloseregeneratfasern, die beispielsweise im Lyocell-Prozess oder im Viskoseverfahren hergestellt werden, sind heute von großer Bedeutung für die Herstellung textiler Erzeugnisse. Trotz des frühen wirtschaftlichen Erfolgs war die genaue Struktur der Cellulose noch bis in die 1920er Jahre ungeklärt, bis Hermann Staudinger den Grundstein zur Polymerchemie legte. ${ }^{18,19}$

Heute wissen wir, dass Cellulose ein unverzweigtes Polymer ist und aus $\beta$-1,4-glycosidisch verknüpften D-Glucose-Einheiten (AGU: anhydroglucose unit) besteht, die in der thermodynamisch bevorzugten ${ }^{4} \mathrm{C}_{1}$-Konformation vorliegen. Um die bevorzugten Bindungswinkel der Acetalbrücken einhalten zu können, ist jede zweite AGU um $180^{\circ}$ in der Ebene gedreht. Je zwei nebeneinanderliegende Struktureinheiten entsprechen dem Disaccharid Cellobiose (Abbildung 3). Der Polymerisationsgrad (DP: degree of polymerization) kann je nach Herkunft der Cellulose über 10000 betragen. ${ }^{19}$

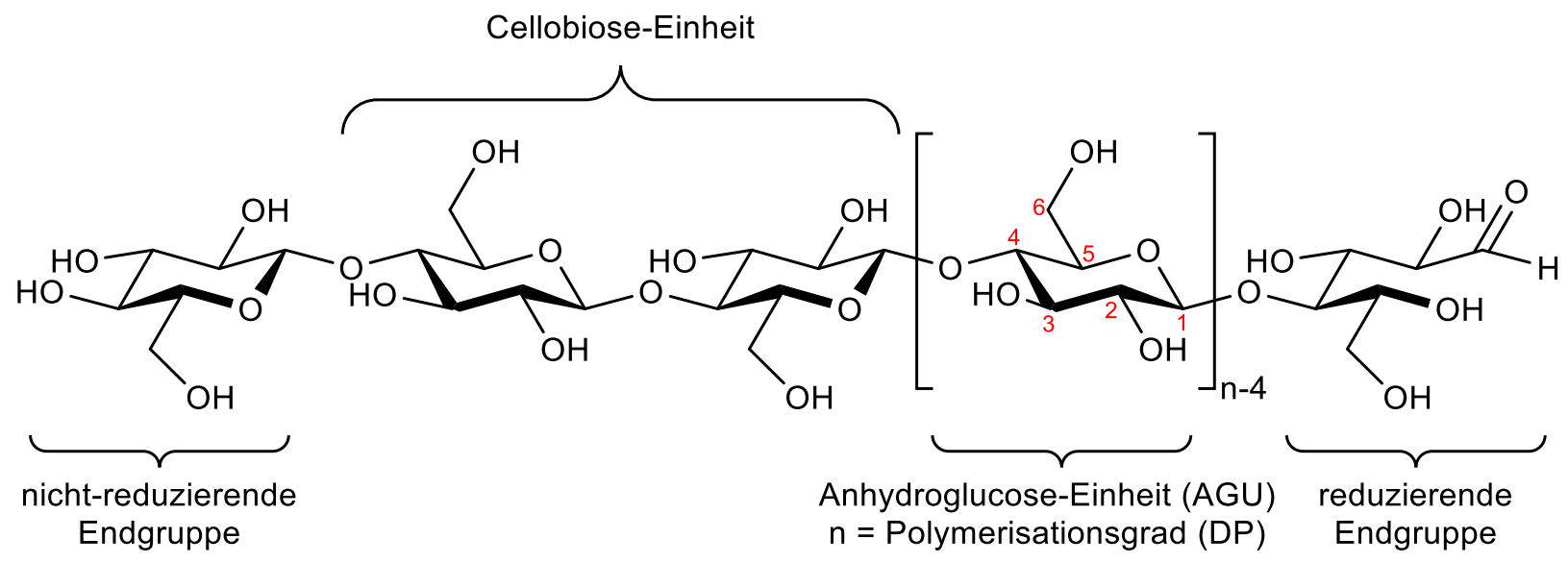

Abbildung 3: Molekulare Struktur der Cellulose. ${ }^{20}$

Jede AGU besitzt insgesamt drei Hydroxylgruppen, zwei sekundäre an den Kohlenstoffatomen C2 und C3 sowie eine primäre an C6. Die Hydroxylgruppen bilden ein intra- und intermolekulares Netzwerk an Wasserstoffbrücken. Cellulose ist teilkristallin und besteht aus ungeordneten amorphen Bereichen, die hoch geordnete kristalline Bereiche unterbrechen. ${ }^{21}$ Durch diese molekulare Struktur erhält Cellulose ihre Eigenschaften, wie zum Beispiel eine hohe thermische Stabilität, und bildet hierarchische und supramolekulare Strukturen aus. 
Durch die drei Hydroxylgruppen pro AGU ist auch die chemische Variabilität groß, da diese in unterschiedlichsten Reaktionen modifiziert werden können. In der Polysaccharidchemie wird üblicherweise der durchschnittliche Substitutionsgrad (DS: degree of substitution) verwendet, der angibt, wie viele der drei Hydroxylgruppen einer einzelnen AGU modifiziert wurden. Folglich liegt der DS immer zwischen 0 und 3, muss aber nicht zwingend ganzzahlige Werte annehmen, da das Cellulosepolymer aus vielen AGUs besteht, die unterschiedlich modifiziert sein können. ${ }^{20}$

Wenn Cellulose funktionalisiert werden soll, kann dies heterogen durchgeführt werden. Hierbei wird die Cellulose im Reaktionsmedium dispergiert. Die Reaktion findet bevorzugt an den amorphen Regionen der Cellulose statt. Reagenzien, die nicht in kristalline Teile eindringen und diese aufbrechen können, können nicht mit diesen reagieren. ${ }^{21}$ Daraus kann eine modifizierte Cellulose resultieren, deren Polymerketten unregelmäßig modifiziert sind.

Eine gleichmäßigere Modifikation des Celluloserückgrates kann dadurch gewährleistet werden, dass die Cellulose vor Reaktionsbeginn gelöst wird und die einzelnen Polymerketten dadurch voneinander getrennt werden. Häufig angewandt in der Synthesechemie sind nicht-wässrige und nichtderivatisierende Lösungsmittel, wie beispielsweise die Kombination aus dem organischen Lösungsmittel $N, N$-Dimethylacetamid (DMAc) mit dem Salz Lithiumchlorid, das die Wasserstoffbrückenbindungen aufbricht und damit den Lösungsvorgang ermöglicht. ${ }^{18}$ Dieses Lösungsmittel ist farblos und das Lösen erfolgt ohne oder unter vernachlässigbarem Kettenabbau, was das Lösungsmittelsystem DMAc/LiCl für unterschiedliche Modifikationen der Cellulose prädestiniert. Die Reaktivität der Hydroxylgruppen in diesem Lösungsmittel nimmt in der Reihenfolge C6 > C2 > C3 $\mathrm{ab}^{18}$

Die bisher gängigsten Methoden zur Funktionalisierung von Cellulose sind Veresterungs- und Veretherungsreaktionen. ${ }^{22}$ Die Click-Chemie erlaubt die Synthese von neuartigen Cellulosederivaten. ${ }^{23}$ Um die Cellulose mittels CuAAC modifizieren zu können, muss diese entweder alkin- oder azidfunktionalisiert werden. Ein Alkin kann in die Cellulose durch eine Veretherung mit Propargylbromid oder durch nukleophile Substitution von Tosylgruppen mit Propargylamin eingebracht werden. ${ }^{22,24,25}$ 


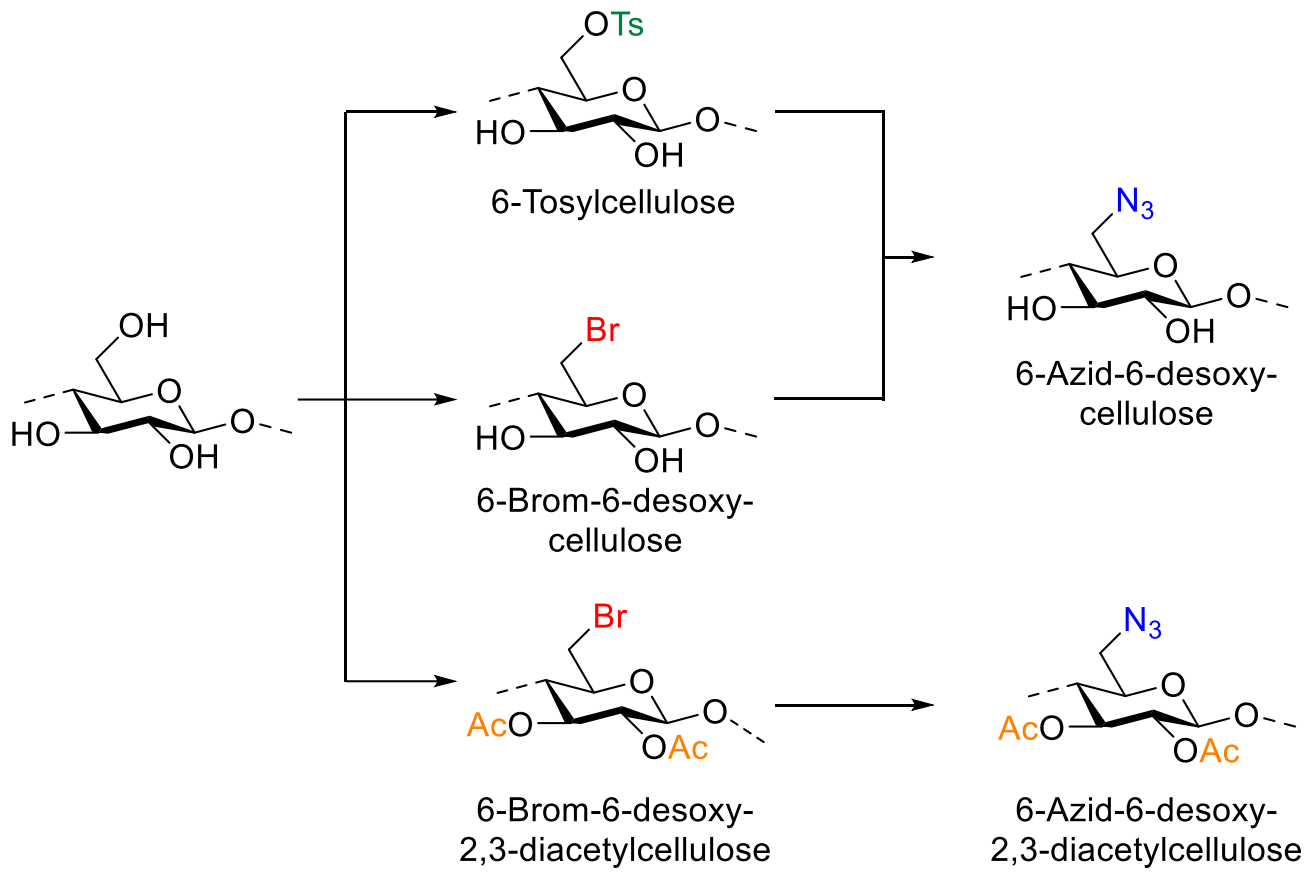

Abbildung 4: Synthese von azidfunktionalisierten Cellulosederivaten.

Häufiger realisiert wird jedoch das Funktionalisieren von Cellulose mit Azidgruppen (Abbildung 4). Da das Azid nicht direkt in die Cellulose eingebracht werden kann, wird ein zweistufiger Reaktionsprozess durchgeführt, bei dem die Cellulose zuerst mit einer guten Abgangsgruppe versehen wird, die dann im nachfolgenden Reaktionsschritt durch Azidgruppen substituiert wird. Eine gängige Abgangsgruppe ist der $p$-Toluensulfonsäureester, der durch Veresterung von Cellulose mit $p$-Toluensulfonsäurechlorid (Tosylchlorid) in DMAc/LiCl hergestellt werden kann. ${ }^{26}$ Der DS kann hierbei über die Menge an zugegebenem Tosylchlorid eingestellt werden. Die Tosylierung geschieht vorzugsweise an C6 und in einem deutlich geringeren Ausmaß an C2 und C3. Tosylcellulose mit $\mathrm{DS}_{\mathrm{Ts}_{\mathrm{s}}}=1$ zeigt eine gute Löslichkeit in N,N-Dimethylformamid (DMF), DMSO und DMAc, was eine einfach durchzuführende nukleophile Substitution unter homogenen Bedingungen erlaubt. ${ }^{23,27}$ Ein alternativer Weg ist die Bromierung mit $\mathrm{N}$-Bromsuccinimid (NBS) und Triphenylphosphin $\left(\mathrm{PPh}_{3}\right)$. Dies führt zu einem Produkt mit hervorragender Regioselektivität. ${ }^{28}$ Der Mechanismus der Bromierungsreaktion verläuft über ein Oxophosphoniumion als Intermediat, das in einer $S_{N} 2$-Reaktion substituiert wird. Da eine solche Reaktion bevorzugt an primären Hydroxylgruppen abläuft, wird ausschließlich C6 substituiert und der $\mathrm{DS}_{\mathrm{Br}}$ kann maximal 1 erreichen. ${ }^{29}$ Die erhaltene 6-Brom-6-desoxycellulose ist in vielen gängigen organischen Lösungsmitteln unlöslich, kann jedoch für die nukleophile Substitution in DMSO aufgequollen werden. Um die Löslichkeit zu verbessern können die beiden verbleibenden Hydroxylgruppen der 6-Brom-6-desoxycellulose im gleichen Reaktionsschritt wie die Bromierung verestert werden. ${ }^{27}$ Nachteilig ist hierbei jedoch, dass C2 und C3 nicht mehr zur weiteren Modifikation zur Verfügung stehen, da sie nun Acetyl-, Propionyl- oder Butyrylgruppen tragen. 
Überwiegend bevorzugt wird der Syntheseweg über die 6-Tosylcellulose zum 6-Azid-6-desoxycellulosederivat. Die anschließende CuAAC wird typischerweise in einem DMSO/Wasser-Gemisch durchgeführt. Als Katalysatorsystem wird ein Kupfer(II)-salz, wie beispielsweise Kupfer(II)-sulfat-Pentahydrat verwendet, das mit Hilfe von Natriumascorbat zur katalytisch wirksamen Kupfer(I)-spezies reduziert wird. ${ }^{23-25,30-35}$ Dieses Katalysatorsystem ist jedoch nicht dazu geeignet, wasser- oder sauerstoffempfindliche Gruppen an die Cellulose anzubringen. Der Einsatz eines Kupfer(I)-salzes in Kombination mit Aminbasen könnte dieses Problem lösen. Um die Kupfer(I)-kalysierte Azid-Alkin-Cycloaddition für die Cellulosemodifikation zu verwenden, ist auch die Synthese eines geeigneten Cellulosederivates nötig, das sich in den dafür verwendeten organischen Lösungsmitteln löst.

\subsection{Kupfer-katalysierte Azid-Alkin-Cycloaddition an Monosacchariden}

Viele Monosaccharide sind von großer biologischer Bedeutung. D-Glucose bildet die Grundeinheit für Biopolymere wie Glycogen, Stärke und Cellulose. Für lebende Organismen ist sie zudem essenziell als Energielieferant und Grundgerüst für anabole Stoffwechselvorgänge. Auch andere Aldohexosen wie D-Galactose und D-Mannose sind physiologisch bedeutsam. ${ }^{36}$ Dies macht die Modifikation von Monosacchariden für die organische Synthese interessant, da die hierbei gewonnenen Erkenntnisse bei der Beantwortung biochemischer Fragestellungen helfen oder medizinische Anwendungen finden können.

Da sich Monosaccharide relativ einfach mit Azid- und Alkinfunktionen versehen lassen, ist die CuAAC ein geeigneter Weg für die Modifikation von Monosacchariden. Die Umsetzung der alkin-/ azidfunktionalisierten Zuckerderivate mit Hilfe der CuAAC ist auf sehr großes Interesse gestoßen und wurde daher in den letzten zwei Jahrzehnten umfangreich untersucht. So wurden unter anderem Triazol-verknüpfte Disaccharidanaloga synthetisiert, aber auch Saccharidderivate mit anderen Molekülen wie Aminosäuren, Glyco- und Lipopeptiden oder unterschiedlichen Polymeren verknüpft. Auch Reaktionen an Oberflächen, Fullerenen oder die Synthese dendritischer Strukturen ist bekannt. ${ }^{7}$

\subsubsection{Funktionalisierung der Monomere für die CuAAC}

Die für die CuAAC benötigten Alkin- oder Azidfunktionalitäten können auf einfachem Wege mit Syntheseprotokollen eingebracht werden, die schon lange routinemäßig eingesetzt werden. So entstehen mono- oder polyfunktionalisierte Zuckerderivate, die in der CuAAC umgesetzt werden können. Mit dieser Herangehensweise können die Zuckerderivate schnell und einfach über 
heterocyclische Triazol-Ringe als Bindeglied miteinander oder mit anderen Molekülen zu einem größeren Biomolekül zusammengefügt werden. ${ }^{7}$

Je nach Position, an der die Azidgruppe eingefügt werden soll, werden unterschiedliche Protokolle benötigt. Am anomeren Kohlenstoff (C1) können Azide, ausgehend von peracetylierten Monosacchariden (1, Abbildung 5), entweder direkt mit den Reagenzien Trimethylsilylazid und Zinn(IV)-chlorid oder zweistufig über das Bromid ( $\alpha$-Anomer, 2) als Intermediat mit einer darauffolgenden $\mathrm{S}_{\mathrm{N}}$ 2-Reaktion unter Inversion der Konfiguration eingebracht werden. In beiden Fällen wird fast ausschließlich das $\beta$-Anomer (3) erhalten. Neben der peracetylierten Galactose (1, Abbildung 5) können auch andere peracetylierte Monosaccharide wie beispielsweise Glucose auf dem gleichen Weg zum Azid umgesetzt werden. ${ }^{37}$

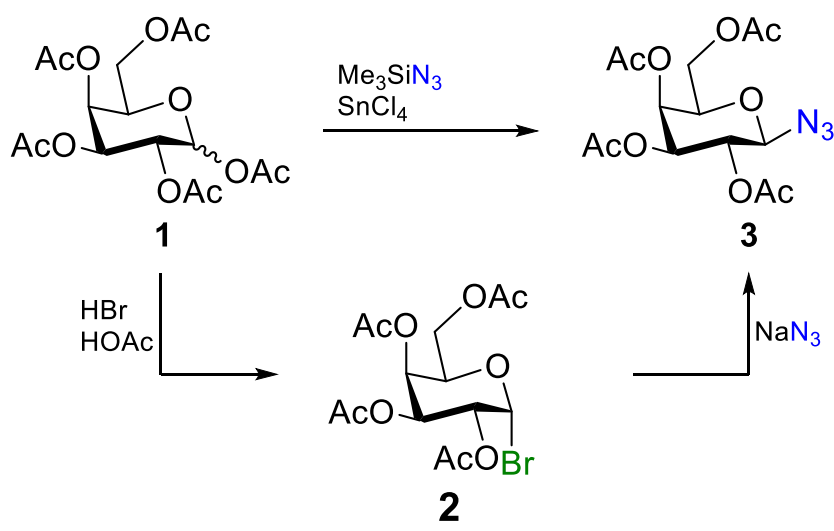

Abbildung 5: Azidfunktionalisierung an C1: Direkte Synthese von 2,3,4,6-Tetra-O-acetyl- $\beta$-Dgalactopyranosylazid (3) ${ }^{38}$ und Synthese über 2,3,4,6-Tetra-O-acetyl- $\alpha$-D-galakcopyranosylbromid (2) ${ }^{37}$.

Um eine Azidgruppe an C6 anzubringen wird die Ausgangsverbindung, wie zum Beispiel Methyl- $\alpha$-Dgalactose (4, Abbildung 6), zuerst mit einer Tosyl-Abgangsgruppe an C6 versehen. Dies ist selektiv möglich, da durch die Wahl der passenden Reaktionsbedingungen nur die primäre und damit reaktivste Hydroxylgruppe mit dem Tosylierungsreagenz reagiert. Nach Peracetylierung der verbleibenden Hydroxylgruppen wird Verbindung $\mathbf{5}$ erhalten, deren Tosylgruppe mit Natriumazid in einer $S_{N}$ 2-Reaktion substituiert wird, wobei ein 6-Azid-6-desoxygalactosederivat erhalten wird.

Auf ähnlichem Wege kann eine Azidfunktion auch an C4 eingebracht werden. Zuerst müssen alle Hydroxylgruppen bis auf die an C4 blockiert werden (7), da die anschließende Veresterung mit Trifluormethansulfonsäureanhydrid an C4 sonst nicht selektiv möglich ist (8). Bei der Triflylgruppe handelt es sich um eine gute Abgangsgruppe, die im nächsten Schritt mit Natriumazid in einer nukleophilen Substitution umgesetzt wird. Im Vergleich zur nukleophilen Substitution an C6 sind hier schärfere Reaktionsbedingungen erforderlich, da die Reaktion an einem sekundären Kohlenstoffatom 
durchgeführt wird. Die $\mathrm{S}_{\mathrm{N}}$ 2-Reaktion verläuft unter Inversion der Konfiguration, was in diesem Beispiel bedeutet, dass das Galactosederivat in ein azidfunktionalisiertes Glucosederivat transformiert wird.
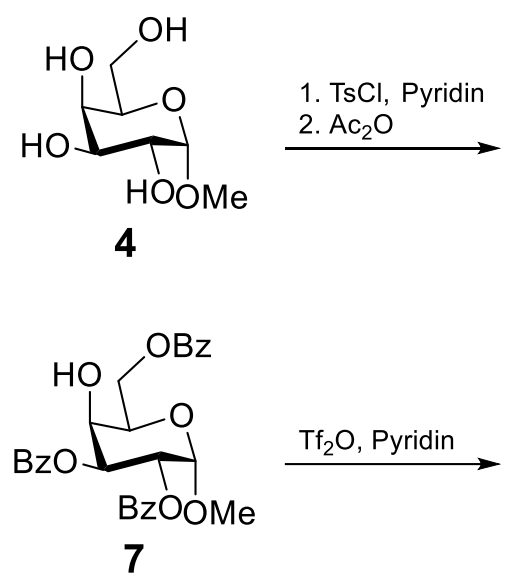

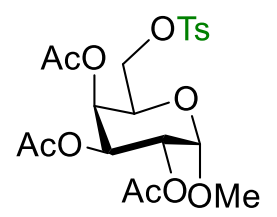

5

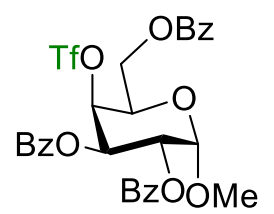

8

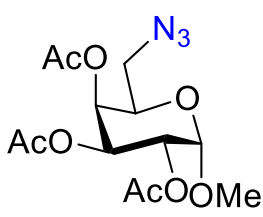

6

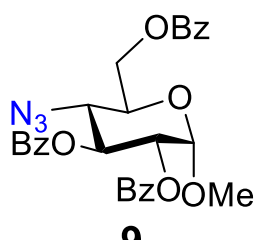

Abbildung 6: Synthese von Monosaccharidderivaten mit Azid an C6 (6) oder an C4 (9). ${ }^{39}$

Eine alternativ oder zusätzlich zur Azidfunktion für die CuAAC benötigte Alkinfunktion kann ebenfalls direkt in ein Monosaccharid eingebracht werden. Selektiv an C1 kann dies unter anderem mit Propargylbromid und Bortrifluoriddiethyletherat durchgeführt werden (10, Abbildung 7). Die Veretherung der anderen Hydroxylgruppen kann mit Natriumhydrid als Base durchgeführt werden. In Abbildung 7 ist die Veretherung an $\mathrm{C} 4$ eines Galactosederivates gezeigt (12).

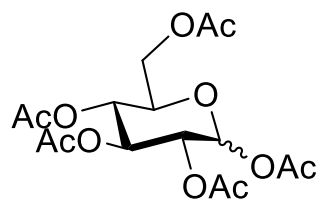

1

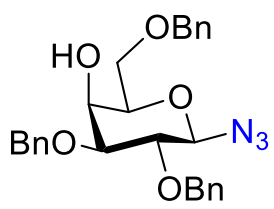

11

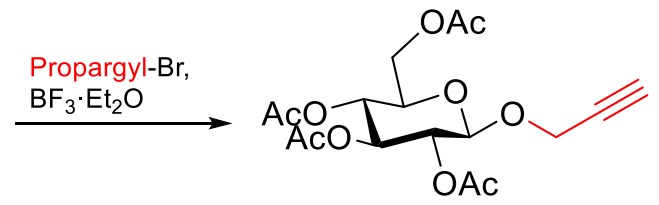

10

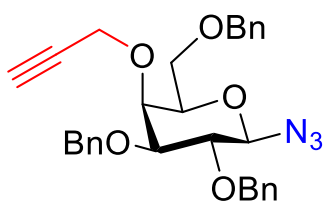

12

Abbildung 7: Alkinfunktionalisierung unterschiedlicher Monosaccharidderivate an C1 (10) ${ }^{40}$ und an C4 (12)

Neben den hier gezeigten Synthesewegen gibt es noch viele weitere Möglichkeiten wie Alkin- oder Azidgruppen in ein Monosaccharidderivat eingebracht werden können. 


\subsubsection{Polymerisation von Monosacchariden mittels CuAAC}

Eine bisher weniger untersuchte Anwendung der CuAAC ist die Polymerisation von funktionalisierten Monosacchariden. Die auf den ersten Blick einfachste Methode ist das Umsetzen bifunktioneller Monosaccharidderivate, also solcher, die sowohl eine Azid- als auch eine Alkinfunktion tragen. Bei der Mehrzahl der bisher basierend auf diesem Verfahren veröffentlichten Reaktionen wurden allerdings hauptsächlich cyclische Produkte gebildet. Zum Beispiel werden bei der CuAAC von 12 (Abbildung 7) Cyclodextrin-Analoga wie das cyclische Dimer, Trimer, Tetramer und weitere höhere cyclische Oligomere erhalten. ${ }^{38}$ Ähnliches Verhalten konnte auch bei mannosebasierten Di- und Trisacchariden beobachtet werden, die hauptsächlich cyclisch dimerisierten. ${ }^{41,42}$ Auch viele andere bifunktionelle Saccharidderivate neigten zur Cyclisierung während der CuAAC. ${ }^{43,44}$ Für die Synthese linearer Polymere stellt dieses Verhalten der Monomere allerdings ein Problem dar.

Über die erfolgreiche Synthese linearer Triazol-verknüpfter Saccharidanaloga gibt es bisher nur wenige Berichte. Das Galactosederivat 12 (Abbildung 7) konnte beispielsweise in einer topochemischen Huisgen-Cycloaddition zum 1,5-Triazol-verknüpften Polygalactosid polymerisiert werden. Voraussetzung dafür war, dass Alkin und Azid im Kristall in einer dem Übergangszustand ähnlichen Geometrie angeordnet sind. ${ }^{45}$ Dies ist jedoch nur bedingt auf andere Monomere übertragbar. Eine Hürde für die Anwendung dieser Polymere stellt auch deren schlechte Löslichkeit in gängigen organischen Lösungsmitteln dar. Beschrieben ist zudem eine konvergente Synthese, bei der ein Triazolverknüpftes Hexadecamannosid in vier Reaktionszyklen aufgebaut wird. ${ }^{46}$ Jedoch ist ein solcher Ansatz streng genommen keine Polymerisation und synthetisch sehr aufwendig, was die Möglichkeit zur Synthese von Polysaccharidanaloga einschränken könnte. Ein weiterer Ansatz ist die CuAAC-Oligo-/ Polymerisation ausgehend von einem Startmolekül, das entweder eine Azid- oder eine Alkingruppe trägt. Dies verhindert die Cyclisierung, da nur ein Kettenende eine CuAAC-reaktive funktionelle Gruppe trägt. Dabei muss jedoch mit einem schwer zu trennenden Produktgemisch gerechnet werden, das auch cyclische Nebenprodukte aus der Reaktion der Monomere untereinander enthält. ${ }^{47}$ 


\section{Fragestellung und Zielsetzung}

Das Hauptziel dieser Arbeit ist die Entwicklung neuer Methoden, mit denen Kohlenhydrate in der wasser- und sauerstofffreien Cul/DIPEA-katalysierten Azid-Alkin-Cycloaddition in organischen Lösungsmitteln umgesetzt werden können.

1. In der Cellulosechemie werden vorherrschend Kupfer(II)-salze in Kombination mit einem Reduktionsmittel in wässrigen Lösungen oder Mischungen von Wasser mit organischen Lösungsmitteln wie beispielsweise DMSO zur Katalyse der Azid-Alkin-Cycloaddition genutzt. Mit diesem Katalysatorsystem ist es jedoch nicht möglich, wasser- oder sauerstoffempfindliche Substrate mit Cellulosederivaten in der CUAAC umzusetzen. Die Entwicklung einer Cul/DIPEA basierten Methode zur effizienten Modifikation von Cellulose würde daher die Möglichkeiten zur Cellulosemodifikation mittels CuAAC erheblich erweitern.

Das Ziel des ersten Teils meiner Arbeit ist infolgedessen die effiziente Synthese eines Cellulosederivates, das in der CUAAC mit Cul/DIPEA in wasserfreien organischen Lösungsmitteln umgesetzt werden kann. Im Anschluss soll die erfolgreiche Cul/DIPEA-katalysierte Azid-AlkinCycloaddition an diesem Cellulosederivat demonstriert werden (Manuskript 1).

2. Auch wenn sich manche azid- und alkinfunktionalisierten Monosaccharidderivate gut in Wasser lösen, so besteht in wässrigen Lösungsmitteln die Gefahr der Agglomeration ihrer Reaktionsprodukte aus der CuAAC, was sich nachteilig auf den Reaktionsverlauf auswirken könnte. Die Durchführung solcher Reaktionen in organischen Lösungsmitteln stellt möglicherweise eine Lösung für dieses Problem dar.

Ziel des zweiten Teils meiner Arbeit ist daher die Entwicklung einer Methode zur Herstellung linearer Polymere aus Monosaccharidderivaten mittels Cul/DIPEA-katalysierter Azid-AlkinCycloaddition. Dafür nötig ist auch die Entwicklung eines geeigneten alkin- und azidfunktionalisierten Monosaccharidderivates. Bisher war die Polymerisation von bifunktionellen Monosaccharidderivaten zu linearen Polymeren nicht möglich, da die Cyclisierung der Monomere als Hauptreaktion dominierte. Daher ist die Minimierung cyclischer Nebenprodukte eine der Hauptherausforderungen. (Manuskript 2+3). 


\section{Ergebnisse und Diskussion}

\subsection{CuAAC an Cellulose}

Um Cellulose in der Cul/DIPEA-katalysierten Azid-Alkin-Cycloaddition nutzen zu können müssen Azidoder Alkingruppen am Celluloserückgrat vorhanden sein. Außerdem muss sich das Cellulosederivat in organischen Lösungsmitteln, wie beispielsweise THF, lösen. Die Anforderung an die Löslichkeit wird vom bisher gebräuchlichen Cellulosederivat 6-Azid-6-desoxycellulose nicht erfüllt. Aufgrund dessen war die Entwicklung und Synthese eines neuen Cellulosederivates nötig.

Die Löslichkeit in organischen Lösungsmitteln könnte durch das Einbringen von Trimethylsilylgruppen (TMS-Gruppen) gewährleistet werden. Infolgedessen wurde die Synthese eines azidfunktionalisierten Cellulosederivates angestrebt, dessen verbleibende Hydroxylgruppen mit TMS-Gruppen geschützt sind.

\subsubsection{Synthese von 6-Azid-6-desoxy-2,3-bis(trimethylsilyl)cellulose}

Im ersten Schritt musste eine Abgangsgruppe eingebracht werden, die zu einem späteren Zeitpunkt gegen Azidgruppen nukleophil substituiert werden kann. Begonnen wurde mit der Synthese von 6-Brom-6-desoxycellulose (13, Abbildung 8). Mikrokristalline Cellulose (MCC) wurde hierfür in DMAc/LiBr gelöst und mit NBS/PPh ${ }_{3}$ nach der Vorschrift von MATSUI ET AL. ${ }^{28}$ selektiv an C6 mit DS $\mathrm{Br}_{\mathrm{B}}=0,9$ bromiert.

Der nächste Schritt war die Silylierung der verbleibenden Hydroxylgruppen der 6-Brom-6-desoxycellulose. Die Silylierung wurde, wie von RICHTER UND KLEMM ${ }^{48}$ beschrieben, homogen in DMAc/LiCl und mit Hexamethyldisilazan (HMDS) sowie einer katalytischen Menge Trimethylsilylchlorid (TMSCl) durchgeführt. Die Reaktionszeit betrug zwei Tage bei Raumtemperatur und zwei Tage bei $100^{\circ} \mathrm{C}$. Während der Reaktion fiel das Cellulosederivat aus und die Reaktion wurde heterogen zu Ende geführt. Die protonenkernresonanzspektroskopische ( $\left.{ }^{1} \mathrm{H}-\mathrm{NMR}\right)$ Analyse des isolierten Reaktionsprodukts ergab einen $\mathrm{DS}_{\mathrm{TMS}}=3$. Daraus lässt sich folgern, dass die Silylierung unter vollständigem Verlust des Broms an C6 ablief (15, Abbildung 8). Daher wurden alternative Reaktionsbedingungen für die Silylierung erprobt. Als neues Lösungsmittel wurde die ionische Flüssigkeit 1-Butyl-3-methylimidazoliumacetat (BmimAc) gewählt. ${ }^{49}$ Dadurch ging das Lösen von 13 schneller und einfacher von statten. Außerdem waren kürzere Reaktionszeiten von nur einem Tag möglich, da das während der Reaktion ausgefallene Produkt durch Zugabe von Toluen wieder gelöst werden konnte und die Reaktion somit homogen zu Ende geführt wurde. Auch bei der Reaktion in der ionischen Flüssigkeit wurde der vollständige Verlust des Broms beobachtet. Zudem lag die Ausbeute 
unter $10 \%$ und eine schwache Hydroxylbande im Infrarotspektrum deutete darauf hin, dass die Hydroxylgruppen nicht restlos umgesetzt wurden. Eine mögliche Erklärung für die schlechte Ausbeute sind Nebenreaktionen und der Abbau der Cellulose, was durch Verunreinigungen der ionischen Flüssigkeit oder des Edukts begünstig werden könnte.

Da die Synthesen mit den Bromid-Abgangsgruppen an C6 nicht zum gewünschten Cellulosederivat führten, wurde ein alternativer Syntheseweg über Tosyl-Abgangsgruppen angestrebt. 6-Tosylcellulose $\left(\mathbf{1 6}\right.$, Abbildung 8) konnte wie von RAHN ET AL. ${ }^{26}$ beschrieben mit $\mathrm{DS}_{\mathrm{Ts}}=0,8-0,9$ synthetisiert werden. Im Vergleich zum bromierten Cellulosederivat löst sich 6-Tosylcellulose in DMAc. Daher wurde die Silylierung mit HMDS/TMSCl ohne LiCl-Zusatz durchgeführt. Schließlich kam es auch bei der Silylierung von 6-Tosylcellulose zum partiellen Verlust der Tosyl-Abgangsgruppe. Aus dem Schwefelgehalt der Verbindung, der mittels Elementaranalyse bestimmt wurde, sowie ${ }^{1} \mathrm{H}-\mathrm{NMR}$ Daten wurde bestimmt, dass ein Produkt mit $\mathrm{DS}_{\mathrm{Ts}_{\mathrm{s}}}=0,4$ und $\mathrm{DS}_{\mathrm{TMS}}=2,6$ synthetisiert wurde. Das erhaltene Produkt 17 löst sich gut in THF und quillt nur in DMSO und DMAc. Da die möglichst vollständige Modifikation von C6 mit Abgangsgruppen gewünscht war und mit $\mathrm{DS}_{\mathrm{Ts}_{\mathrm{s}}}=0,4$ nicht erreicht wurde, wurde keine nukleophile Substitution mit dieser Verbindung durchgeführt.

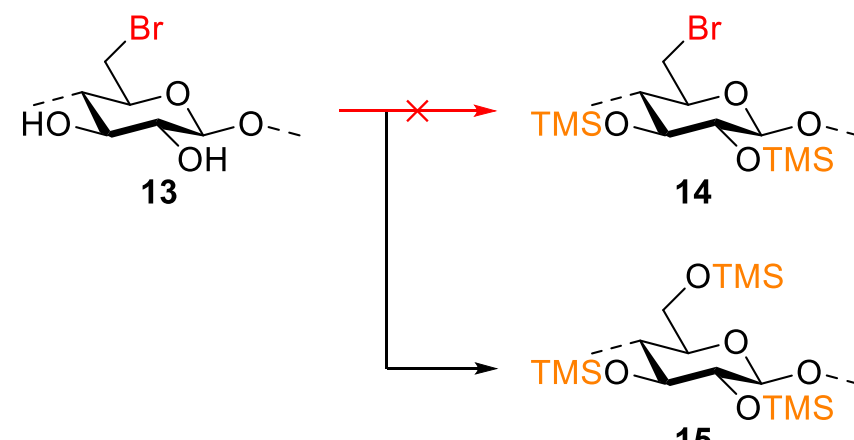

15

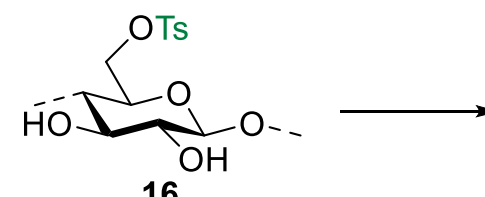

16

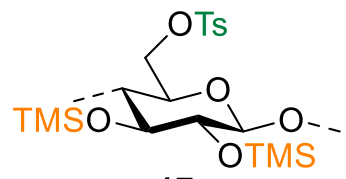

17

$\mathrm{DS}_{\mathrm{Ts}}=0,8 \quad \mathrm{DS}_{\mathrm{Ts}}=0,4$

Abbildung 8: Silylierung der Cellulosederivate 6-Brom-6-desoxycellulose (13) und 6-Tosylcellulose (16).

In Manuskript 1 (Seite 34) wird eine Eintopfreaktion beschrieben, bei der Bromierung und Silylierung der MCC (Abbildung 9) in nur einem Schritt durchgeführt wurden. Hierbei wurde die MCC in DMAc/LiBr gelöst, mit NBS/PPh 3 versetzt und kurz darauf wurde HMDS/TMSCl zugegeben, ohne dass das Zwischenprodukt 6-Brom-6-desoxycellulose isoliert wurde. Mit steigendem DS TMS $_{\text {fiel das }}$ 
Reaktionsprodukt während der Reaktion als feines Pulver aus und die Reaktion wurde heterogen zu Ende geführt. Vorteilhaft bei dieser Methode ist das schnelle Aufarbeiten des Reaktionsproduktes, das durch Filtration aus der Reaktionslösung gewonnen und durch Waschen und Rühren in Methanol gereinigt werden konnte. Aufgrund der hohen Oberfläche des Pulvers löst sich das Produkt schnell und gut in Chloroform und THF und quillt in DMSO, was nachfolgende Reaktionen sowie die Analytik erleichterte.
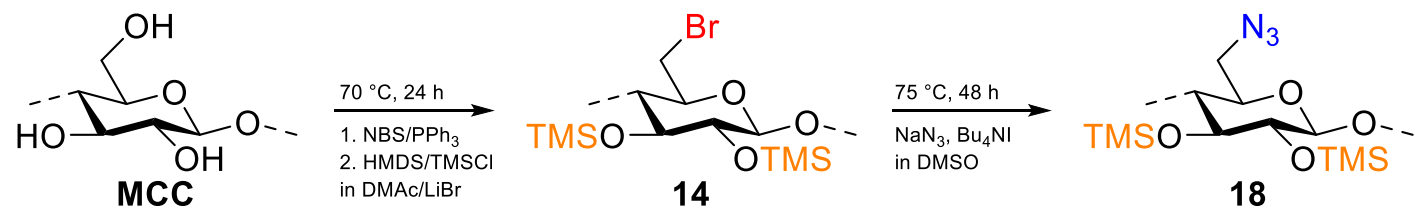

Abbildung 9: Synthese von 6-Azid-6-desoxy-2,3-bis(trimethylsilyl)cellulose (18) über 6-Brom-6-desoxy-2,3bis(trimethylsilyl)cellulose (14).

Um die besten Reaktionsbedingungen zu identifizieren wurde die Reaktionszeit der Bromierung zwischen einer und zwei Stunden variiert und es wurden entweder 2,5 äq. oder 4 äq. Bromierungsreagenz zugesetzt. Es konnte gezeigt werden, dass die Silylierung nach 24 Stunden bei $70{ }^{\circ} \mathrm{C}$ vollständig abgeschlossen ist, da in Infrarotspektren der Produkte keine Hydroxylbande mehr detektiert werden konnte. Daher konnte von der vollständigen Substitution aller Hydroxylgruppen ausgegangen werden. Der DS ${ }_{\text {TMS }}$ wurde aus ${ }^{1} \mathrm{H}-\mathrm{NMR}$-Spektren bestimmt. Indirekt daraus wurde der $\mathrm{DS}_{\mathrm{Br}}=3-\mathrm{DS}_{\mathrm{TMS}}$ berechnet. Außerdem wurde die Molmassenverteilung mittels GPC bestimmt. Die Messergebnisse sind in Form des zahlengemittelten Polymerisationsgrades DP $_{n}$, des gewichtsgemittelten Polymerisationsgrades $\mathrm{DP}_{\mathrm{w}}$ und der Dispersität $Ð$ in Tabelle 1 gezeigt.

Der erhaltene $D S_{B r}$ liegt zwischen $D S_{B r}=0,3$, wenn die Bromierung nur eine Stunde mit 2,5 äq. $\mathrm{NBS} / \mathrm{PPh}_{3}$ durchgeführt wurde, und $\mathrm{DS}_{\mathrm{Br}}=0,89$ bei zwei Stunden Reaktionszeit mit 4 äq. Bromierungsreagenz (Tabelle 1). Bei den Reaktionsprodukten mit 4 äq. Bromierungsreagenz waren Ausbeuten und $\mathrm{DP}_{\mathrm{w}}$ geringer als bei ihren Pendants, die mit 2,5 äq. NBS/PPh ${ }_{3}$ hergestellt wurden. Außerdem zeigten diese eine rötliche oder bräunliche Verfärbung, was auf unerwünschte Nebenreaktionen und Verunreinigungen im Produkt hindeutet.

Das beste Ergebnis wurde mit 2,5 äq. Bromierungsreagenz und zwei Stunden Reaktionszeit für die Bromierung erzielt, da mit $\mathrm{DS}_{\mathrm{Br}}=0,8$ ein hoher Bromierungsgrad bei einer guten Ausbeute von $80 \%$ erreicht wurde. Dieses Cellulosederivat wurde, wie in Abbildung 9 gezeigt, weiter zu 6-Azid-6-desoxy-2,3-bis(trimethylsilyl)cellulose (18) umgesetzt. Da 14 in DMSO nur aufquillt und sich nicht löst, waren lange Reaktionszeiten in der nukleophilen Substitution von 48 Stunden nötig. So 
wurde ein $D S_{\text {Azid }}=0,7$ erreicht und etwas Brom $\left(D S_{B r}=0,1\right)$ verblieb im Molekül. Außerdem fand eine leichte Hydrolyse von durchschnittlich 0,2 TMS-Gruppen pro AGU statt. Das Cellulosederivat 18 löst sich in THF und quillt in DMSO, DMF und Toluol. Da an Polymere gebundene Azide zur Reaktion mit Dichlormethan und Chloroform unter Bildung von explosivem Di- und Triazidomethan neigen, konnten diese Lösungsmittel nicht verwendet werden. ${ }^{50}$ Die Bestimmung der Molekulargewichtsverteilung mittels GPC war mit diesem Cellulosederivat nicht möglich, da das in THF gelöste Polymer zu hochviskos war, um es durch einen PTFE-Spritzenfilter $(0,45 \mu \mathrm{m})$ zu filtrieren.

Tabelle 1: Übersicht über die Analysenergebnisse der Optimierung der Eintopfreaktion.

\begin{tabular}{|c|c|c|c|c|c|}
\hline Reaktionszeit & & $1 \mathrm{~h}$ & $2 \mathrm{~h}$ & $1 \mathrm{~h}$ & $2 \mathrm{~h}$ \\
\hline$\left[\mathrm{NBS}+\mathrm{PPh}_{3}\right]$ & & 2,5 äq. & 2,5 äq. & 4 äq. & 4 äq. \\
\hline \multirow[t]{2}{*}{${ }^{1} \mathrm{H}-\mathrm{NMR}$} & $\mathrm{DS}_{\text {TMS }}$ & 2,70 & 2,20 & 2,51 & 2,11 \\
\hline & $\mathrm{DS}_{\mathrm{Br}}$ & 0,30 & 0,80 & 0,49 & 0,89 \\
\hline \multirow[t]{3}{*}{ GPC* } & $\mathrm{DP}^{*}{ }^{*}$ & 50,3 & 36,9 & 51,1 & 39,4 \\
\hline & $\mathrm{DP}_{\mathrm{w}} *$ & 176,7 & 107,5 & 130,9 & 98,2 \\
\hline & $\bigoplus=D P_{w} / D P_{n}$ & 3,51 & 2,91 & 2,56 & 2,49 \\
\hline Ausbeute & & $79 \%$ & $80 \%$ & $71 \%$ & $65 \%$ \\
\hline
\end{tabular}

* GPC wurde in THF vs. Polystyrolstandard gemessen. $\mathrm{DP}_{\mathrm{n}}$ und $\mathrm{DP}_{\mathrm{w}}$ wurden durch Division der Messergebnisse durch die mittleren molaren Massen der AGUs berechnet und sind daher nicht als Absolutwert zu verstehen, sondern nur zum Vergleich untereinander gedacht.

Kurz zusammengefasst kann gesagt werden, dass 6-Azid-6-desoxy-2,3-bis(trimethylsilyl)cellulose mit $\mathrm{DS}_{\text {Azid }}=0,7$ synthetisiert wurde. Dieses azidfunktionalisierte Cellulosederivat ist THF-löslich und kann damit als Ausgangsverbindung für die Cul/DIPEA-katalysierte Azid-Alkin-Cycloaddition genutzt werden.

\subsubsection{CuAAC an 6-Azid-6-desoxy-2,3-bis(trimethylsilyl)cellulose}

Umgesetzt wurde 6-Azid-6-desoxy-2,3-bis(trimethylsilyl)cellulose in der Cul/DIPEA - katalysierten AzidAlkin-Cycloaddition mit dem aromatischen Phenylacetylen zu 19 und dem aliphatischen 3-Cyclopentyl1-propin zu 20 (Abbildung 10). Die daraus resultierenden Polymere tragen am neu erzeugten TriazolRing entweder Phenylgruppen (Ph) oder Methylen(cyclopentyl)gruppen (cPe). Bei beiden ClickReaktionen konnte eine geringe Hydrolyse der TMS-Gruppen auf DS $_{\text {TMS }} \sim 1,65$ beobachtet werden.

Nach der Reaktion von 18 mit 3-Cyclopentyl-1-propin kann die im Edukt sehr stark ausgeprägte Azidbande im Infrarotspektrum des Produktes nicht mehr detektiert werden und aus dem ${ }^{1} \mathrm{H}$-NMR- 
Spektrum konnte ein $\mathrm{DS}_{\mathrm{CPe}}=0,7$ bestimmt werden, was den vollständigen Umsatz der Azidgruppen des Cellulose-Eduktes beweist. Der $\mathrm{DP}_{\mathrm{n}}(\mathrm{GPC})$ ist auf 17 gesunken mit einer Dispersität $Ð=1,51$.

Im Infrarotspektrum des Click-Produktes mit Phenylacetylen verblieb nach 7 Tagen Reaktionszeit noch eine schwache Azidbande. Die Auswertung des Protonen-Kernresonanzspektrums dieser Verbindung bewies den unvollständigen Umsatz der Azidgruppen und ergab einen $D S_{\mathrm{Ph}}=0,42$. Die GPC-Messung war mit diesem Cellulosederivat nicht möglich. Der höhere Umsatz in der Reaktion mit 3-Cyclopentyl1-propin könnte auf den geringeren sterischen Anspruch des Methylen(cyclopentyl)-Restes am Alkin zurückzuführen sein, im Vergleich zur größeren aromatischen Phenylgruppe des Phenylacetylens.

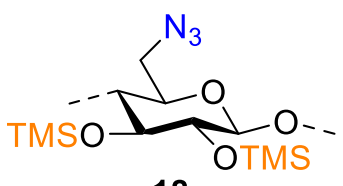

18
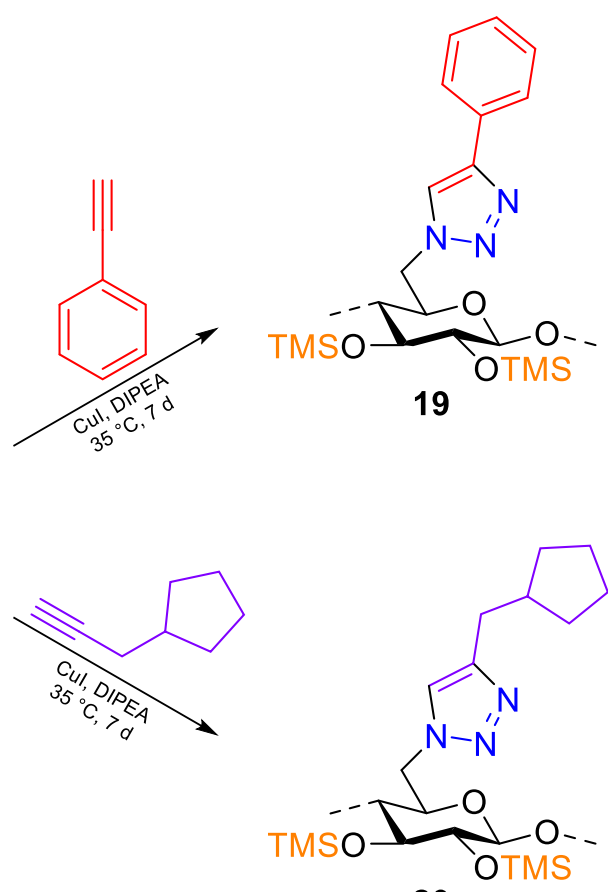

20

Abbildung 10: Cul/DIPEA-katalysierte Azid-Alkin-Cycloaddition am Cellulosederivat 18 mit aromatischem Phenylacetylen zu 19 und aliphatischem 3-Cyclopentyl-1-propin zu 20.

\subsubsection{Diskussion und Folgerung}

Zuerst wurde die Synthese von 6-Brom-6-desoxy-2,3-bis(trimethylsilyl)cellulose aus MCC in einer Eintopfreaktion gezeigt. Die schnelle und effiziente Synthese, zeitsparende Aufarbeitung sowie die durch TMS-Gruppen bedingte gute Löslichkeit in Chloroform und THF machen dieses Cellulosederivat zu einem hervorragenden Ausgangsmaterial für unterschiedliche Anwendungen. Dies könnten beispielsweise eine Grignard-Reaktion oder palladiumkatalysierte Kreuzkupplungen sein. Möglich ist auch die hier gezeigte nukleophile Substitution an C6. 
Von Fox UND EDGAR ${ }^{27}$ wurden ähnliche Cellulosederivate beschrieben, die regioselektiv an $\mathrm{C} 6$ bromiert und mit Acetyl-, Propionyl- oder Butyryl-Gruppen an C2 und C3 verestert sind. Im Vergleich zu den hier gezeigten TMS-geschützten Cellulosederivaten lösen sich diese veresterten Derivate auch in DMF, DMSO und Aceton. Jedoch könnten Ester bei gewissen Reaktionen wie beispielsweise einer GrignardReaktion stören. Außerdem lassen sie sich nicht so leicht wie TMS-Gruppen hydrolysieren. ${ }^{51}$ Dadurch ergibt sich ein anderes Anwendungsspektrum für diese Derivate. Wenn Folgereaktionen an C2 und C3 geplant sind, könnte das TMS-geschützte Cellulosederivat die bessere Ausgangsverbindung darstellen.

Des Weiteren wurde hier die nukleophile Substitution des Broms mit Azidgruppen gezeigt, die zum Cellulosederivat 6-Azid-6-desoxy-2,3-bis(trimethylsilyl)cellulose führte. Der Umsatz dieser Reaktion lag bei $88 \%$. Hierfür wurden die gleichen Reaktionsbedingungen gewählt, die auch für die nukleophile Substitution von 6-Brom-6-desoxycellulose mit Natriumazid beschrieben wurden. ${ }^{28}$ Dort wurde die gleiche Ausbeute von ca. 80 \% sowie auch die beinahe quantitative Substitution des Broms berichtet. Dies legt den Schluss nahe, dass sich die Anwesenheit der TMS-Gruppen an C2 und C3 nicht negativ auf den Ablauf der nukleophilen Substitution auswirkte. Es kann angenommen werden, dass mit etwas erhöhter Reaktionszeit oder Reaktionstemperatur auch der quantitative Umsatz in der nukleophilen Substitution erreicht werden könnte.

Im nächsten Schritt wurde mit diesem Cellulosederivat die grundlegende Machbarkeit der CuAAC an Cellulose unter wasser- und sauerstofffreien Bedingungen exemplarisch sowohl mit einem aromatischen als auch einem aliphatischen Alkin gezeigt. Im Vergleich zu bisherigen Arbeiten in Wasser/DMSO-Lösungen mit einem Katalysatorsystem aus einem Kupfer(II)-salz und Natriumascorbat können in der Cul/DIPEA-katalysierten Methode auch wasserunlösliche oder sauerstoffempfindliche Substrate eingesetzt werden.

Auch wenn die Reaktionen erfolgreich verlaufen sind, deuten die Reaktionszeit von einer Woche sowie der unvollständige Umsatz in einer der beiden Reaktionen darauf hin, dass noch Optimierungspotential bei den Reaktionsbedingungen besteht. Dies lässt auch den Schluss zu, dass das gewählte Katalysatorsystem Cul/DIPEA möglicherweise nur bedingt für den hier gezeigten Einsatz geeignet ist. Verbessert werden könnte der Prozess durch die Zugabe von Tris[(1-benzyl-1H-1,2,3triazol-4-yl)methyl]amin (TBTA). Dieser Ligand ist dafür bekannt, dass er die Reaktionsgeschwindigkeit erhöht und gleichzeitig den Oxidationszustand Kupfer(I) stabilisiert. ${ }^{52}$

\subsection{CuAAC-Polymerisation eines Monosaccharidderivates}

Wird die Synthese eines linearen Polymers mittels CuAAC-Polymerisation angestrebt, so kann entweder mit diazid- und dialkinfunktionalisierten Monomeren (AA/BB-System) gearbeitet werden, 
oder mit nur einem Monomer, das beide funktionellen Gruppen in einem Molekül trägt (ABMonomer).

Bei der Polymerisation von asymmetrischen AA/BB-Monomeren kann es zu Kopf-Schwanz-, Kopf-Kopfund Schwanz-Schwanz-Verknüpfungen zweier Wiederholeinheiten im Polymer kommen. Folglich wird ein weniger regelmäßiges Polymer erhalten, als dies bei Homopolymerisation eines AB-Monomers der Fall ist, da hierbei ausschließlich Kopf-Schwanz-Verknüpfungen möglich sind. Da monosaccharidbasierte Monomere asymmetrisch sind, wird der AB-Ansatz bevorzugt, um ein möglichst regelmäßiges Polymer zu erhalten.

\subsubsection{Synthese eines geeigneten monosaccharidbasierten Monomers}

Zuerst musste ein geeignetes Monosaccharid gewählt werden, an das die für die Polymerisation benötigten Azid- und Alkingruppen angebracht werden können. Literaturbekannt ist schon das Galactosederivat 12 (Abbildung 11). Es wurde berichtet, dass die CuAAC dieses Derivates cyclische Dimere (15\%), Trimere (22\%), Tetramere (15\%), Pentamere (5\%) sowie höhere cyclische Oligomere (33\%) liefert. ${ }^{38}$ Lineare Oligo-/Polymere wurden bei der CUAAC mit diesem Monomer nicht erhalten. Erklärt wurde dies über die Molekülgeometrie. Der Winkel zwischen den in Abbildung 11 grün markierten Bindungen beträgt ungefähr $90^{\circ}$ beim Galactosederivat 12. Durch diese Orientierung wird die Bildung cyclischer Produkte begünstigt. Daraus kann die Hypothese abgeleitet werden, dass bei der Polymerisation eines Monomers mit einem Winkel von rund $180^{\circ}$ zwischen diesen grün markierten Bindungen (Abbildung 11) ein höherer Anteil linearer Produkte erhalten wird. Bei Glucose- oder Mannosederivaten ist die Hydroxylgruppe an C4 äquatorial angeordnet, was zu dem Winkel von ca. $180^{\circ}$ zwischen diesen Bindungen führt. Daher könnten sich diese beiden Monosaccharide als Ausgangsmaterial für die Monomersynthese eignen. Die Polymerisation des Glucosederivates 21 würde zu einem der Cellulose ähnelnden Polymer führen, bei dem die $\beta$-1-4-glykosidische Bindung

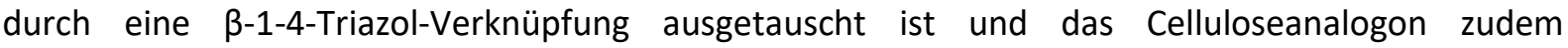
perbenzyliert ist. Daher wurde die Synthese des Glucosederivates 21 angestrebt.

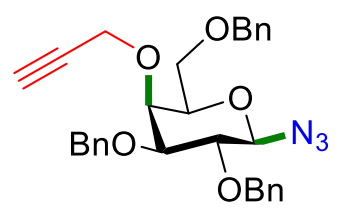

12

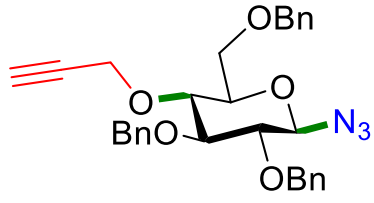

21

Abbildung 11: Azid- und alkinfunktionalisierte Galactose- (12) und Glucosederivate (21). 
Das schon literaturbekannte Konzept ${ }^{38}$ zur Synthese von 12 wurde für die Synthese des Monomers 21 angepasst und optimiert. Die achtstufige Synthese von 21 ist erstmals in Manuskript 2 (ab Seite 51) beschrieben. Sie wurde mit einer Gesamtausbeute von $37 \%$ durchgeführt, was einer durchschnittlichen Ausbeute von $88 \%$ pro Reaktionsschritt entspricht.

\subsubsection{CuAAC-Oligomerisation an der Oberfläche von Silica-Nanopartikeln}

Im nächsten Schritt wurde eine Methode entwickelt, mit der das glucosebasierte Monomer 21 polymerisiert werden konnte (Manuskript 2). Um sicherzugehen, dass lineare Ketten erhalten werden, wurde ein Startmolekül verwendet, das ausschließlich Azidgruppen trug. Als ein solches Startmolekül wurden Silica-Nanopartikel (SiNPs) gewählt. Diese bieten den Vorteil, dass sie zum einen chemisch sehr stabil sind und zum anderen inklusive der auf ihrer Oberfläche gebundenen linearen Polymere leicht aus der Reaktionslösung durch Zentrifugation abtrennbar sind. Alle weiteren Produkte, wie beispielsweise cyclische Oligomere, verbleiben in der überstehenden Lösung und können separat analysiert werden. Im Vergleich mit einem niedermolekularen Startmolekül bot dieses Vorgehen den Vorteil, dass keine Trennverfahren wie HPLC oder GPC benötigt werden. ${ }^{47}$

Bisher wurden kommerziell erhältliche Bromalkylsilane verwendet, um Bromgruppen auf der Oberfläche der SiNPs einzuführen, die im Anschluss gegen Azidgruppen substituiert wurden. Jedoch gestaltet sich die Überwachung des Reaktionsfortschritts der $S_{N} 2$-Reaktion auf der Oberfläche der SiNPs generell als schwierig, wodurch es nicht einfach ist, die vollständige Substitution des Broms sicherzustellen. ${ }^{53}$ Daher wurde ein neuer Silanlinker entworfen und synthetisiert, der eine endständige Azidgruppe trägt (30, Abbildung 12). Dieser Silanlinker wurde auf der Oberfläche sphärischer Nanopartikel angebracht, die einen Durchmesser von ca. $50 \mathrm{~nm}$ aufweisen. Jede an die Oberfläche gebundene Kette der SiNPs-N $\mathbf{N}_{3}$ trägt genau eine endständige Azidgruppe.

Der Erfolg der Oberflächenfunktionalisierung mit Azidgruppen wurde durch die Elementaranalyse bestätigt. Aus dem Stickstoffgehalt von 0,46 Gew.-\% der Nanopartikel SiNPs-N $\mathbf{N}_{\mathbf{3}}$ konnte ein Azidgehalt von 109,5 $\mathrm{mmol} / \mathrm{g}$ berechnet werden, der sich mit Hilfe der BET-Oberfläche von 49,25 m²/g zu einer Graftingdichte von $2,22 \mu \mathrm{mol} / \mathrm{m}^{2}$ umrechnen ließ. Um diese Zahlen besser einschätzen zu können wurden sie mit Ergebnissen anderer Studien verglichen. Bei den dort gezeigten Experimenten mit Silanlinkern, die sterisch minimal anspruchsvoller sind, wurden Graftingdichten zwischen 1,9 und 2,0 $\mu \mathrm{mol} / \mathrm{m}^{2}$ erreicht. ${ }^{54,55}$ Daraus kann abgeleitet werden, dass die hier gezeigte Reaktion hervorragend funktioniert hat und keiner weiteren Optimierung bedarf. 


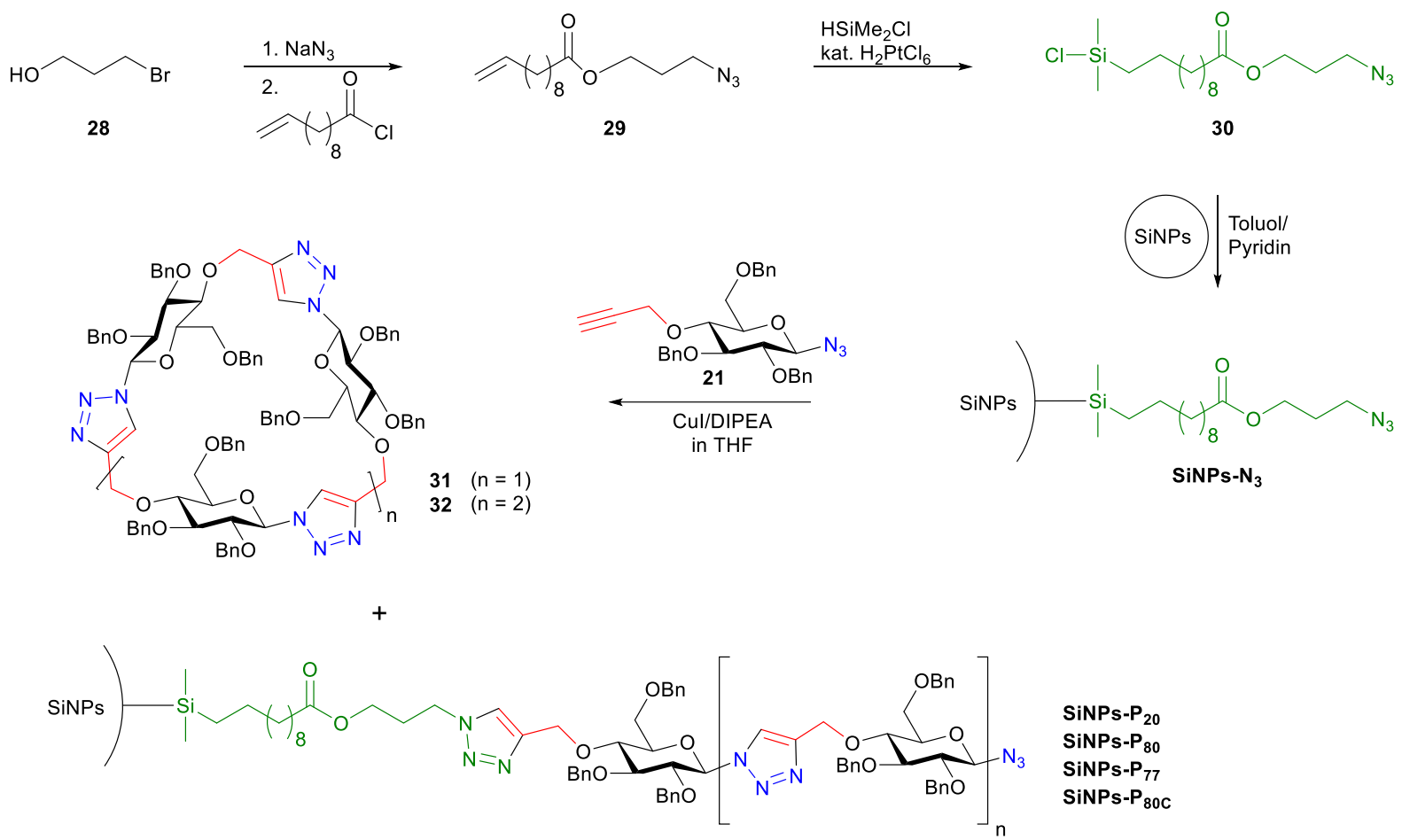

Abbildung 12: Synthese des Monochlorsilan-Linkers 30, der azidfunktionalisierten Silica-Nanopartikel SiNPs-N $\mathbf{3}$ und die nachfolgende CuAAC mit dem Monomer $\mathbf{2 1}$ an deren Oberfläche, die je nach gewählten

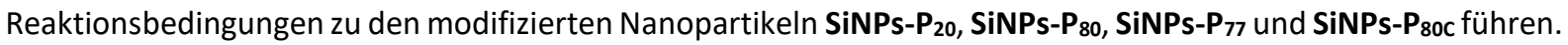

Im Anschluss wurde das Monomer 21 gemeinsam mit den SiNPs- $\mathbf{N}_{\mathbf{3}}$ in der Cul/DIPEA-katalysierten Azid-Alkin-Cycloaddition umgesetzt. Im Zuge der Optimierung der Reaktion wurden insgesamt vier unterschiedliche Reaktionsbedingungen getestet. Die unterschiedlich modifizierten SiNPs wurden nach der zugegeben Monomermenge benannt (SiNPs-P $\mathbf{2 0}_{\mathbf{2 0}}$, SiNPs-P $_{\mathbf{8 0}}$, SiNPs-P $_{\mathbf{7 7}}$, SiNPs-P $_{\mathbf{8 0}}$ ). Nach dem Abschluss der Reaktion wurden die Nanopartikel von der Reaktionslösung durch Zentrifugation abgetrennt und gewaschen. Aus den Daten der Elementaranalyse wurde der durchschnittliche Polymerisationsgrad auf der Oberfläche berechnet. Die überstehende Lösung wurde eingedampft und die darin enthaltenen Verbindungen wurden MALDI-massenspektrometrisch und NMRspektroskopisch untersucht (Tabelle 2).

Mit 20 äq. Monomer 21 konnte nur ein DP von 3,46 auf der Oberfläche der SiNPs-P $\mathbf{2}_{\mathbf{2}}$ erzielt werden, was einer Ausbeute von 17,3 \% auf der Oberfläche entspricht. Das restliche Monomer cyclisierte in der überstehenden Lösung und bildete hauptsächlich das Trimer sowie in geringem Maße das Tetramer. Die Höhe des Signals des Tetramers beträgt im MALDI-Massenspektrum nur 5 \% der Signalhöhe des Trimer-Signals. 
Tabelle 2: Elementare Zusammensetzung der SiNPs-N3 und SiNPs nach der Reaktion mit 21 (SiNPs-P $\mathbf{2 0}, \mathbf{S i N P s}_{\mathbf{3}}-\mathbf{P}_{\mathbf{8 0}}$, SiNPs-P ${ }_{77}$, SiNPs-P $_{80 c}$ ) in Gew.-\%, der daraus berechnete Polymerisationsgrad DP, Ausbeute auf der Oberfläche (OF) sowie das Verhältnis der Signalhöhen im MALDI-Massenspektrum von Tetramer 32 zu Trimer 31.

\begin{tabular}{lllllll}
\hline Probe & $\mathrm{C}$ & $\mathrm{H}$ & $\mathrm{N}$ & $\mathrm{DP}$ & Ausbeute & $\begin{array}{l}\text { Signalverhältnis } \\
\text { auf OF }\end{array}$ \\
& & & & & & $\mathbf{3 2}$ zu $\mathbf{3 1}$ \\
\hline SiNPs-N & 3,50 & 1,20 & 0,46 & - & - & - \\
SiNPs-P20 & 13,90 & 1,86 & 1,73 & 3,46 & $17,3 \%$ & $5 \%$ \\
SiNPs-P80 & 21,30 & 2,45 & 2,57 & 6,62 & $8,3 \%$ & $19 \%$ \\
SiNPs-P77 & 24,80 & 2,70 & 2,96 & 8,44 & $11,0 \%$ & $15 \%$ \\
SiNPs-P80 & 31,75 & 3,24 & 3,73 & 13,01 & $16,3 \%$ & 0 \\
\hline
\end{tabular}

Um den Polymerisationsgrad an der Oberfläche der SiNPs- $\mathbf{N}_{3}$ weiter zu erhöhen, wurde die Reaktion im nächsten Experiment unter den gleichen Reaktionsbedingungen mit der vierfachen Monomerkonzentration durchgeführt, also mit 80 äq. Monomer. Trotz des vierfachen Monomereinsatzes stieg der DP nur um 91 \% auf 6,62 (SiNPs-P8) und die Ausbeute auf der Oberfläche sank auf 8,3\%. Der Anteil des gebildeten Tetramers stieg stark an. Dieses Ergebnis zeigt, dass durch die Erhöhung der Monomerkonzentration die Bildung cyclischer Oligomere bevorzugt wird. Im Umkehrschluss bedeutet dies, dass eine geringere Monomerkonzentration zu einem höheren Polymerisationsgrad an der Oberfläche führen sollte.

Um dies zu bestätigen wurde das Monomer schrittweise zugegeben. Stündlich wurden 11 äq. Monomer zugegeben bis insgesamt 77 äq. zugesetzt wurden. Hierdurch wurde die durchschnittliche Monomerkonzentration während der Reaktion verringert, obwohl die beinahe gleiche Menge an Monomer zugesetzt wurde. Dies erhöhte den erhaltenen Polymerisationsgrad auf der Oberfläche der SiNPs-P ${ }_{77}$ auf 8,44 und der Anteil des cyclischen Tetramers in der überstehenden Lösung ging zurück. Um die aktive Monomerkonzentration während der Polymerisation weiter zu verringern, wurden im nächsten Experiment 80 äq. Monomer kontinuierlich über einen Zeitraum von 24 Stunden zugegeben. Dies wurde mit einer Spritzenpumpe realisiert. Der Polymerisationsgrad auf den resultierenden SiNPs-P80 stieg auf 13,01 und die Ausbeute entsprach beinahe der aus dem ersten Experiment mit 20 äq. Monomer (16,3\% vs. 17,3\%). Außerdem wurde ausschließlich das cyclische Trimer in der überstehenden Lösung gebildet.

Es kann davon ausgegangen werden, dass eine weitere Verringerung der aktiven Monomerkonzentration zu noch höheren Polymerisationsgraden auf der Oberfläche führt. 


\subsubsection{CuAAC-Polymerisation an endfunktionalisiertem Polyethylenglycol}

Die im vorhergehenden Kapitel entwickelte Methode wurde in Manuskript 3 (Seite 76) angewandt, um das Monomer 21 an den Kettenenden von propargylfunktionalisertem Polyethylenglycolmethylether (34a, Abbildung 13, $\mathrm{M}_{\mathrm{n}} \approx 2000 \mathrm{~g} / \mathrm{mol}$ ) und propargylfunktionalisertem Polyethylenglycol (34b, $M_{n} \approx 2000 \mathrm{~g} / \mathrm{mol}$ ) zu polymerisieren. Es wurden 50 oder 80 Äquivalente des Monomers über einen Zeitraum von je 48 Stunden zudosiert. Auf diesem Weg wurden vier unterschiedliche Blockcopolymere hergestellt, zwei Diblock-Copolymere pseudo-Cellulose-block-PEG $\left(\mathbf{D}_{50}\right.$ und $\left.\mathbf{D}_{\mathbf{8 0}}\right)$ und zwei TriblockCopolymere pseudo-Cellulose-block-PEG-block-pseudo-Cellulose ( $\mathbf{T}_{50}$ und $\left.\mathbf{T}_{\mathbf{8 0}}\right)$.<smiles>CC(C)([SeH])CO[TlH]</smiles>

33a $\quad \mathrm{R}=\mathrm{Me}$

33b $\quad \mathrm{R}=\mathrm{H}$<smiles>C#CCCBr</smiles>

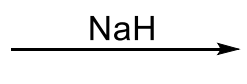

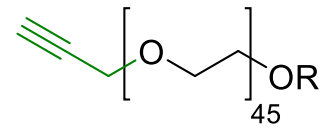

34a $\quad \mathrm{R}=\mathrm{Me}$

34b $\mathrm{R}=\mathrm{CH}_{2} \mathrm{C} \equiv \mathrm{CH}$

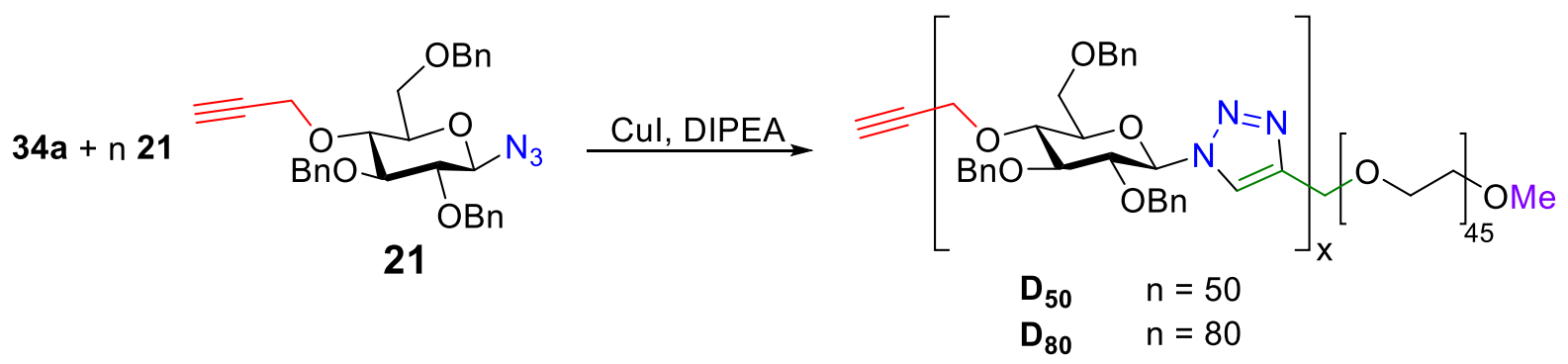

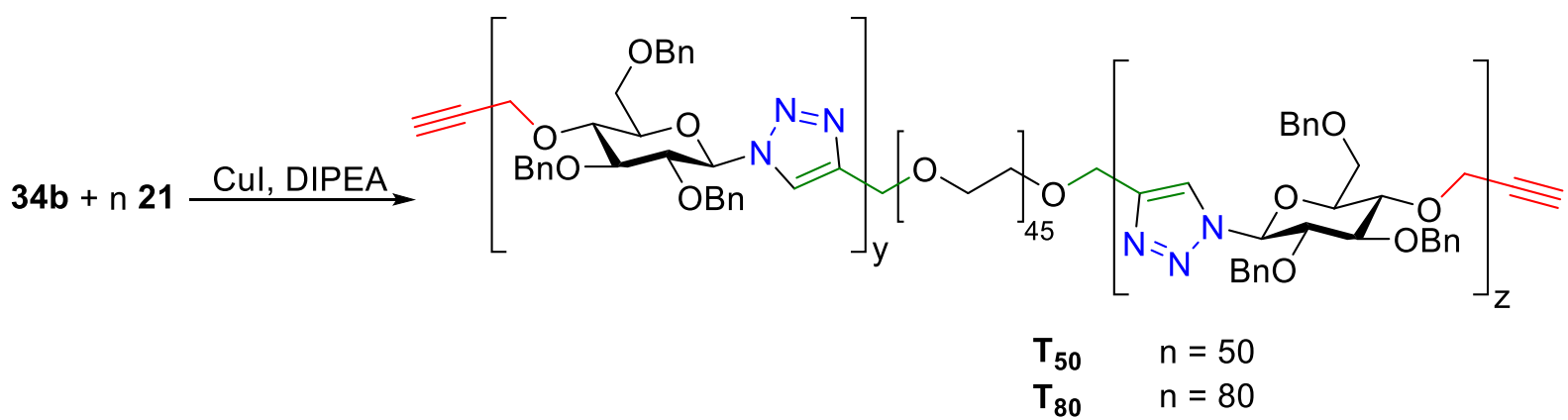

Abbildung 13: Synthese von alkinfunktionalisierten PEG-Derivaten (34a und 34b) und anschließende CuAACPolymerisation des bifunktionellen Glucosederivates 21 an den Kettenenden von 34a und 34b zu DiblockCopolymeren ( $D_{50}$ und $D_{80}$ ) und Triblock-Copolymeren ( $T_{50}$ und $T_{80}$ ).

Die Ausbeute lag bei allen Reaktionen zwischen $88 \%$ und $94 \%$. Nach keiner dieser Polymerisationsreaktionen konnte die Entstehung von Trimeren, Tetrameren oder anderen Oligomeren in MALDI-Massenspektren nachgewiesen werden. Der Polymerisationsgrad der DiblockCopolymere konnte NMR-spektroskopisch auf $D_{n}(N M R)=48\left(D_{50}\right)$ und $75\left(D_{80}\right)$ bestimmt werden. 
Hierfür wurde das Signal der Methoxy-Endgruppe des PEGs mit dem Signal des Protons an C1 des pseudo-Cellulose-Blocks verglichen. Bei der Verwendung des Signals der Propargylendgruppen zur DPBestimmung wurden $\mathrm{zu}$ hohe Werte erhalten. Dies deutet auf eine partielle Zersetzung der Propargylendgruppen hin. Das Signal der PEG-Protonen kann nicht zur Bestimmung des DPs genutzt werden, da dies von den Signalen der Protonen an C5 und C6 des pseudo-Saccharidblocks überlagert ist. Daher war die NMR-spektroskopische Bestimmung des Polymerisationsgrades bei den TriblockCopolymeren ( $\mathbf{T}_{50}$ und $\left.\mathbf{T}_{80}\right)$ nicht möglich.

Um die Molekulargewichtsverteilung der synthetisierten Polymere zu ermitteln wurden GPCMessungen durchgeführt. Die Polymere $D_{50}$ und $T_{50}$ weisen eine Dispersität $\oslash\left(M_{w} / M_{n}\right)$ von rund 2,1 auf und die der Polymere $\mathbf{D}_{\mathbf{8 0}}$ und $\mathrm{T}_{80}$ beträgt ca. 2,5 (Tabelle 3). Ihr $\mathrm{M}_{\mathrm{n}}$ bewegt sich zwischen 11,5 und $16,5 \mathrm{~kg} / \mathrm{mol}$

Die erhaltenen Polymere sind Amphiphile. Der PEG-Block ist in allen Blockcopolymeren gleich groß und löst sich gut in polaren Lösungsmitteln wie Wasser, Methanol und Ethanol, aber auch in vielen weiteren organischen Lösungsmitteln wie THF, Chloroform und Toluol. Die Größe der pseudoCellulose-Blöcke ist variabel und abhängig von den Reaktionsbedingungen der Synthese, und sie lösen sich nur in unpolaren Lösungsmitteln wie THF oder Chloroform. Aufgrund dieser Eigenschaften konnten die Polymere nach Größe getrennt werden. Das Block-Copolymer wurde hierbei in einem definierten Volumen THF gelöst, welches ein gutes Lösungsmittel für beide Blöcke ist, und es wurde ein definiertes Volumen Methanol zugegeben, welches ausschließlich für den PEG-Block ein gutes Lösungsmittel ist. Dabei fiel die Fraktion der Block-Copolymere aus, deren Löslichkeit durch einen großen pseudo-Cellulose-Block bedingt wurde. Diese Fraktionen haben ein hohes Molekulargewicht und wurden $\mathbf{D}_{50 \mathrm{H}}, \mathbf{D}_{80 \mathrm{H}}, \mathbf{T}_{50 \mathrm{H}}$ und $\mathbf{T}_{80 \mathrm{H}}$ genannt. Der $\mathrm{DP}_{\mathrm{n}}(\mathrm{NMR})$ der beiden Diblock-Copolymer-Fraktionen $\left(D_{50 \mathrm{H}}\right.$ und $\left.\mathbf{D}_{\mathbf{8 0 H}}\right)$ liegt zwischen 123 und 148. GPC-Messungen ergaben ein $M_{n}=22,1-30,4 \mathrm{~kg} / \mathrm{mol}$ aller gefällter Polymerfraktionen, während deren Dispersität auf $€=1,41-1,88$ verringert wurde. $\mathbf{D}_{\mathbf{8 0 H}}$ und $\mathbf{T}_{\mathbf{8 0 H}}$ wurden in höherer Ausbeute als ihre Pendants aus der Polymerisation mit 50 äq. Monomer $\left(\mathbf{D}_{50 \mathrm{H}}\right.$ und $\mathbf{T}_{50 \mathrm{H}}$ ) erhalten.

Die Polymerfraktion mit geringerem Molekulargewicht und kleineren pseudo-Cellulose-Blöcken blieb gelöst und konnte durch Eindampfen des Lösungsmittels gewonnen werden ( $\left.\mathbf{D}_{\mathbf{5 0 L}}, \mathbf{D}_{\mathbf{8 0 L}}, \mathbf{T}_{\mathbf{5 0 L}}, \mathbf{T}_{\mathbf{8 0}}\right)$. Diese sind mit $M_{n}=6,3-8,8 \mathrm{~kg} / \mathrm{mol}$ deutlich kleiner und mit Dispersität $\oslash=1,41-1,49$ gleichmäßiger. Der $\mathrm{DP}_{\mathrm{n}}(\mathrm{NMR})$ der Diblock-Copolymerfraktionen liegt zwischen 21 und 27. 
Tabelle 3: Übersicht über Zahlenmittel der Molmasse $\left(M_{n}\right)$, Gewichtsmittel der Molmasse $\left(M_{w}\right)$ und die daraus berechnete Dispersität $Ð$, die aus GPC-Messungen bestimmt wurden, über die Ausbeute der Polymere aus der Polymerisationsreaktion und die Ausbeuten der einzelnen Fraktionen nach Trennung und über den Polymerisationsgrad, der aus ${ }^{1} \mathrm{H}-\mathrm{NMR}$-Spektren berechnet wurde.

\begin{tabular}{|c|c|c|c|c|c|}
\hline Probe & $\mathrm{Mn}^{\mathrm{a}}(\mathrm{kg} / \mathrm{mol})$ & $\mathrm{M}_{\mathrm{w}}{ }^{\mathrm{a}}(\mathrm{kg} / \mathrm{mol})$ & $Ð\left(M_{w} / M_{n}\right)$ & Ausbeute (\%) & $D P_{n}{ }^{b}$ \\
\hline$D_{50}$ & 11,5 & 24,4 & 2,12 & 94 & 48 \\
\hline $\mathrm{D}_{50 \mathrm{~L}}$ & 8,3 & 12,3 & 1,49 & 34 & 27 \\
\hline $\mathrm{D}_{5 \mathrm{OH}}$ & 30,4 & 42,8 & 1,41 & 55 & 148 \\
\hline$D_{80}$ & 12,4 & 30,6 & 2,48 & 90 & 75 \\
\hline $\mathrm{D}_{80 \mathrm{~L}}$ & 6,5 & 9,2 & 1,41 & 25 & 21 \\
\hline $\mathrm{D}_{8 \mathrm{OH}}$ & 24,0 & 40,3 & 1,68 & 68 & 123 \\
\hline $\mathrm{T}_{50}$ & 13,9 & 28,6 & 2,05 & 93 & n.m. ${ }^{c}$ \\
\hline $\mathrm{T}_{50 \mathrm{~L}}$ & 8,8 & 12,7 & 1,45 & 25 & n.m. ${ }^{\mathrm{c}}$ \\
\hline $\mathrm{T}_{5 \mathrm{OH}}$ & 29,4 & 41,3 & 1,41 & 65 & n.m. ${ }^{c}$ \\
\hline $\mathrm{T}_{80}$ & 16,5 & 39,4 & 2,39 & 88 & n.m. ${ }^{c}$ \\
\hline $\mathrm{T}_{80 \mathrm{~L}}$ & 6,3 & 9,0 & 1,43 & 11 & n.m. ${ }^{c}$ \\
\hline $\mathrm{T}_{80 \mathrm{H}}$ & 22,1 & 41,6 & 1,88 & 69 & n.m. ${ }^{\mathrm{c}}$ \\
\hline
\end{tabular}

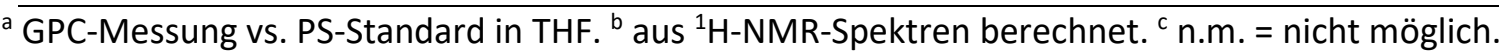

Die Fraktionen mit geringerem Molekulargewicht $\left(\mathbf{D}_{\mathbf{5 0 L}}, \mathbf{D}_{\mathbf{8 0}}, \mathbf{T}_{\mathbf{5 0 L}}, \mathbf{T}_{\mathbf{8 0 L}}\right)$ zeigten Selbstorganisation, wenn sie als THF-Lösung $(5 \mathrm{mg} / \mathrm{ml})$ in einer Wasser/THF-Mischung gefällt wurden und die finale Konzentration auf $0,5 \mathrm{mg} / \mathrm{ml}$ in 1:1 (V/V) Wasser/THF eingestellt wurde. Hierbei wurden sphärische Nanopartikel erhalten. Ihre Größe wurde mittels SEM bestimmt und reichte von $88 \pm 20 \mathrm{~nm}\left(D_{50 \mathrm{~L}}\right)$ über $195 \pm 91 \mathrm{~nm}\left(\mathbf{D}_{80 \mathrm{~L}}\right)$ und $213 \pm 74 \mathrm{~nm}\left(\mathbf{T}_{80 \mathrm{~L}}\right)$ hin zu $243 \pm 63 \mathrm{~nm}\left(\mathbf{T}_{50 \mathrm{~L}}\right)$. Die Nanopartikel, die aus $\mathbf{D}_{50 \mathrm{~L}}$ und $\mathbf{T}_{\mathbf{5 0 L}}$ hergestellt wurden, sedimentierten nach kurzer Zeit, während mit $\mathbf{D}_{\mathbf{8 0}}$ und $\mathbf{T}_{\mathbf{8 0}}$ stabile Dispersionen erzeugt werden konnten.

\subsubsection{Diskussion und Folgerung}

Das Wachstum linearer Ketten und die Bildung cyclischer Verbindungen sind Konkurrenzreaktionen. Um das Produktgemisch dahingehend beeinflussen zu können, eine möglichst hohe Ausbeute linearer Ketten zu erhalten, ist das Verständnis über die Faktoren, die diese Reaktionen beeinflussen, essenziell.

Ein Faktor ist die Reaktionsgeschwindigkeit der beiden Reaktionen, die wiederum von der Konzentration des Startmoleküls und der des Monomers abhängt. Die Reaktionsgeschwindigkeit der Bildung eines linearen Dimers aus zwei Monomeren sollte von der Monomerkonzentration abhängen und die Reaktionsgeschwindigkeit der Reaktion zwischen einem Monomer und dem Startmolekül von der Konzentration der beiden Moleküle. Aus dieser Überlegung lässt sich ableiten, dass mit steigender Monomerkonzentration im Vergleich zur Konzentration des Startmoleküls zunehmend die Bildung 
cyclischer Verbindungen bevorzugt wird. Bestätigt wurde dies durch die Experimente an den SiNPs. Im Idealfall sollte das Startmolekül möglichst reaktiv und die durchschnittliche Monomerkonzentration möglichst gering während der Reaktion sein, um einen möglichst hohen Anteil linearer Verbindungen zu erhalten.

Zudem spielt auch die Geometrie des Monomers eine große Rolle. Experimente zur Synthese kleiner Cyclodextrine aus drei oder vier Glucose-Einheiten haben gezeigt, dass die passende Vororientierung der reaktiven Gruppen für die Bildung solch kleiner cyclischer Verbindungen entscheidend ist. ${ }^{56}$ Cyclische Dimerisierung konnte beim Galactosederivat 12 (Abbildung 11 auf Seite 18) beobachtet werden. ${ }^{38} \mathrm{Im}$ Vergleich mit den eingangs erwähnten Cyclodextrinen gilt es zu beachten, dass das cyclische Dimer des Galactosederivates ungefähr die gleiche Ringgröße wie die Cyclodextrine aus drei bis vier Glucoseinheiten hat, da es insgesamt aus vier Ringen besteht, zwei Pyranoseringen und zwei Triazolringen. Das hier eingesetzte glucosebasierte Monomer 21 unterscheidet sich vom Galactosemonomer durch die Konfiguration von C4. Dies führt dazu, dass das glucosebasierte Monomer nicht cyclisch dimerisieren kann, sondern das Trimer die kleinste cyclische Verbindung darstellt.

Bei genauerer Betrachtung des Produktgemisches der cyclischen Verbindungen nach der Reaktion an den SiNPs fällt auf, dass hauptsächlich das Trimer, wenig Tetramer und keine höheren Oligomere erhalten wurden. Daraus lässt sich folgern, dass die intramolekulare Cyclisierung deutlich schneller als die intermolekularen Reaktionen abläuft. Wäre dies anders herum, so würden wahrscheinlich verstärkt höhere cyclische Oligomere gebildet werden und die Ausbeute an der Oberfläche der SiNPs- $N_{3}$ würde höher ausfallen. Folglich hat auch die Größe der kleinstmöglichen cyclischen Verbindung einen Einfluss auf das Verhältnis von linearen zu cyclischen Produkten. Besser verständlich wird dies anhand eines Beispiels, bei dem ein intermediär gebildetes lineares Dimer betrachtet wird, das an beiden Molekülenden eine CuAAC-reaktive Gruppe trägt. Sofern dieses Molekül in der Lage ist intramolekular zu cyclisieren, würde dies bevorzugt geschehen. Das hierbei gebildete cyclische Dimer kann keine weiteren Reaktionen mehr eingehen. Ist die intramolekulare Dimerisierung nicht möglich, so stehen dem linearen Dimer die Reaktion mit einem weiteren Monomer, linearen Oligomeren oder mit dem wachsenden Polymer am Startmolekül offen. Daraus wird ersichtlich, dass je später die intramolekulare Cyclisierung einsetzt, desto größer die Wahrscheinlichkeit ist, dass das Molekül mit dem wachsenden Polymer am Startmolekül reagiert. Schließlich bedeutet dies, dass je größer die kleinstmögliche cyclische Verbindung ist, die das Monomer bilden kann, desto leichter sollte die Synthese linearer Polymere fallen. Als allgemeine Anforderung an das Monomer könnte folglich formuliert werden, dass die cyclische Dimerisierung des Monomers nicht möglich sein sollte, um möglichst effizient lineare Polymere synthetisieren zu können. Auch die anfangs aufgestellte Hypothese, dass es von Vorteil ist, wenn der Winkel der in Abbildung 11 (Seite 18) grün gefärbten 
Bindungen $180^{\circ}$ zueinander beträgt, konnte mit diesen Experimenten und Ergebnissen bestätigt werden.

Während die Ausbeute an linearen Polymeren auf der Oberfläche der SiNPs auch nach Optimierung der Reaktionsbedingungen nicht über $20 \%$ lag, wurde bei der Polymerisation an PEG eine Ausbeute von über $90 \%$ erreicht. Einzig die Zeit der Zugabe des Monomers wurde von 24 Stunden auf 48 Stunden erhöht, alle anderen Reaktionsbedingungen blieben gleich. Es kann als unwahrscheinlich angesehen werden, dass dies der einzige Faktor für die große Diskrepanz im Syntheseerfolg ist. Möglicherweise ist auch dem Katalysatorsystem Cul/DIPEA ein großer Einfluss zuzuschreiben. Dieses Katalysatorsystem liegt in THF zu großen Teilen dispergiert und nicht gelöst vor, wodurch die Reaktion heterogen katalysiert ist. Dies hat den Vorteil, dass der Katalysator leicht durch Zentrifugation von allen nach der Reaktion gelösten Bestandteilen abgetrennt werden kann. Im Falle der SiNPs wurde das Kupfer(I)-iodid durch Waschen mit Acetonitril entfernt. Damit ist die im Produkt verbleibende Katalysatormenge äußert gering oder nicht vorhanden. Als Nachteil bei der Verwendung dieses Katalysatorsystems stellt sich heraus, dass eine Voraussetzung für die erfolgreiche Verwendung von Cul/DIPEA die gute Löslichkeit aller Reaktionsteilnehmer ist. Da sich die modifizierten SiNPs nicht in THF lösen, sondern nur dispergieren lassen, könnte sich dies negativ auf die Katalyse der Reaktion an deren Oberfläche ausgewirkt haben, nicht jedoch auf die Katalyse in Lösung, die zur Bildung cyclischer Produkte führte. Dies könnte eine Erklärung für die hohe Ausbeute cyclischer Produkte neben den SiNPs und die hervorragend funktionierende Reaktion an endfunktionalisiertem PEG sein.

Es gibt einige Veröffentlichungen, in denen die Synthese vergleichbarer Polymere gezeigt ist, die sich aber in Kernpunkten unterscheiden (vgl. Abschnitt 1.4.2, Seite 10). Ein Ansatz ist die topochemische Polymerisation im Kristall. Dies funktioniert nur, wenn sich das Monomer in einer dem Übergangszustand ähnlichen Orientierung kristallisieren lässt. Dadurch wird die Zahl möglicher Monomere stark eingeschränkt. Auch der Polymerisationsgrad ist nicht einstellbar und die erhaltenen Polymere sind häufig nur schwer löslich. ${ }^{45,57,58}$ Mit einem konvergenten Syntheseansatz konnte ein Triazol-verknüpftes hexadecameres Oligomannosid synthetisiert werden. Der Vorteil dieser Methode ist die perfekte Kontrolle über die Kettenlänge, die allerdings mit einem großen synthetischen Aufwand einhergeht. Die Synthese von Polymeren könnte sich mit dieser Methode als schwierig gestalten, da der Syntheseaufwand mit der gewünschten Kettenlänge zunehmend steigt. ${ }^{46}$

Glucosebasierte Polycarbonate konnten durch Ringöffnungspolymerisation (ROP) cyclischer 4,6-OCarbonyl-glucopyranoside erhalten werden. Mit Ausbeuten von über $90 \%$ und einer Dispersität $\bigoplus<1,2$ konnten sehr regelmäßige Polymere mit $D P_{n}=100$ oder auch Blockcopolymere mit einem Polylactid-Block (PLA) synthetisiert werden. Durch Funktionalisierung des Monomers an C2 und C3 konnten neue Eigenschaften und Funktionen in das Polymer eingebracht werden. ${ }^{59,60}$ Diese ROP ist der 
im Rahmen dieser Arbeit gezeigten CuAAC-Polymerisation in einigen Punkten sehr ähnlich. Beide Methoden erlauben das Erreichen eines hohen Polymerisationsgrades und die Synthese von Blockcopolymeren. Die Modifikation von C2 und C3 des zur CuAAC-Polymerisation verwendeten Monomers 21 sollte analog des Monomers möglich sein, das bei der ROP verwendet wurde. Beide Methoden unterscheiden sich grundlegend in der Art und der Stelle der Verknüpfung. Die ROP scheint auf die Synthese 4,6-Carbonat-verknüpfter Polysaccharidanaloga beschränkt zu sein, während bei der in dieser Arbeit gezeigten CuAAC-Polymerisation 1,4-Triazol-Verknüpfungen erhalten werden. Mit der hier gezeigten Methode ist auch die Verknüpfung der Monomere über andere Stellen denkbar, wenn die Monomere entsprechend designt werden.

Abschließend kann gesagt werden, dass das Katalysatorsystem Cul/DIPEA und die hier entwickelte Methode für die Synthese linearer Polysaccharidanaloga mit löslichen Startmolekülen hervorragend geeignet sind, jedoch nur eingeschränkt für die Synthese von Polysaccharidanaloga an Oberflächen empfohlen werden können. Eingeschränkt wird diese Methode nur durch die beiden Voraussetzungen, dass die Monomere keine cyclischen Dimere bilden können sollten und die Polymerisation mit gut löslichen Ausgangsverbindungen durchgeführt werden sollte. Die hier erhaltenen Ergebnisse legen nahe, dass das Katalysatorsystem Cul/DIPEA auch für die Polymerisation anderer (saccharidbasierter) Monomere verwendbar ist und diese Methode daher sehr breit eingesetzt werden kann. 


\section{Referenzen}

(1) Kolb, H. C.; Finn, M. G.; Sharpless, K. B. Click Chemistry: Diverse Chemical Function from a Few Good Reactions. Angew. Chem., Int. Ed. 2001, 40, 2004-2021.

(2) Anzahl der englischsprachigen Treffer (Journal, Letter, Report, Review) zum Suchbegriff "click chemistry", abgerufen am 22.10.2019 auf scifinder.cas.org.

(3) Huisgen, R. Proceedings of the Chemical Society. October 1961. Proc. Chem. Soc. 1961, 357.

(4) Huisgen, R.; Szeimies, G.; Möbius, L. 1.3-Dipolare Cycloadditionen, XXXII. Kinetik der Additionen organischer Azide an CC-Mehrfachbindungen. Chem. Ber. 1967, 100, 2494-2507.

(5) Tornøe, C. W.; Christensen, C.; Meldal, M. Peptidotriazoles on solid phase: 1,2,3-triazoles by regiospecific copper(i)-catalyzed 1,3-dipolar cycloadditions of terminal alkynes to azides. J. Org. Chem $2002,67,3057-3064$

(6) Rostovtsev, V. V.; Green, L. G.; Fokin, V. V.; Sharpless, K. B. A Stepwise Huisgen Cycloaddition Process: Copper(I)-Catalyzed Regioselective "Ligation" of Azides and Terminal Alkynes. Angew. Chem., Int. Ed. 2002, 41, 2596-2599.

(7) Tiwari, V. K.; Mishra, B. B.; Mishra, K. B.; Mishra, N.; Singh, A. S.; Chen, X. Cu-Catalyzed Click Reaction in Carbohydrate Chemistry. Chem. Rev. 2016, 116, 3086-3240.

(8) Rodionov, V. O.; Presolski, S. I.; Díaz, D. D.; Fokin, V. V.; Finn, M. G. Ligand-accelerated Cu-catalyzed azide-alkyne cycloaddition: a mechanistic report. J. Am. Chem. Soc. 2007, 129, 12705-12712.

(9) Rodionov, V. O.; Fokin, V. V.; Finn, M. G. Mechanism of the ligand-free Cul-catalyzed azide-alkyne cycloaddition reaction. Angew. Chem., Int. Ed. 2005, 44, 2210-2215.

(10) Kuang, G.-C.; Guha, P. M.; Brotherton, W. S.; Simmons, J. T.; Stankee, L. A.; Nguyen, B. T.; Clark, R. J.; Zhu, L. Experimental investigation on the mechanism of chelation-assisted, copper(II) acetateaccelerated azide-alkyne cycloaddition. J. Am. Chem. Soc. 2011, 133, 13984-14001.

(11) Worrell, B. T.; Malik, J. A.; Fokin, V. V. Direct evidence of a dinuclear copper intermediate in Cu(I)catalyzed azide-alkyne cycloadditions. Science 2013, 340, 457-460.

(12) Bock, V. D.; Hiemstra, H.; van Maarseveen, J. H. Cul-Catalyzed Alkyne-Azide "Click" Cycloadditions from a Mechanistic and Synthetic Perspective. Eur. J. Org. Chem. 2006, 2006, 51-68. 
(13) Berg, R.; Straub, B. F. Advancements in the mechanistic understanding of the copper-catalyzed azide-alkyne cycloaddition. Beilstein J. Org. Chem. 2013, 9, 2715-2750.

(14) Mykhalichko, B. M.; Temkin, O. N.; Mys'kiv, M. G. Polynuclear complexes of copper(I) halides: coordination chemistry and catalytic transformations of alkynes. Russ. Chem. Rev. 2000, 69, 957-984. (15) Fazio, F.; Bryan, M. C.; Blixt, O.; Paulson, J. C.; Wong, C.-H. Synthesis of sugar arrays in microtiter plate. J. Am. Chem. Soc. 2002, 124, 14397-14402.

(16) Li, B.; Huang, D.; Qin, A.; Tang, B. Z. Progress on Catalytic Systems Used in Click Polymerization. Macromol. Rapid Commun. 2018, 39, 1800098.

(17) Binauld, S.; Fleury, E.; Drockenmuller, E. Solving the loss of orthogonality during the polyaddition of $\alpha$-azide- $\omega$-alkyne monomers catalyzed by $\mathrm{Cu}(\mathrm{PPh} 3) 3 \mathrm{Br}$ : Application to the synthesis of high-molar mass polytriazoles. J. Polym. Sci., Part A: Polym. Chem. 2010, 48, 2470-2476.

(18) Heinze, T.; Liebert, T. Unconventional methods in cellulose functionalization. Prog. Polym. Sci. $2001,26,1689-1762$.

(19) Klemm, D.; Heublein, B.; Fink, H.-P.; Bohn, A. Cellulose: fascinating biopolymer and sustainable raw material. Angew. Chem., Int. Ed. 2005, 44, 3358-3393.

(20) Klemm, D.; Heinze, T.; Philipp, B.; Wagenknecht, W. New approaches to advanced polymers by selective cellulose functionalization. Acta Polym. 1997, 48, 277-297.

(21) Roy, D.; Semsarilar, M.; Guthrie, J. T.; Perrier, S. Cellulose modification by polymer grafting: a review. Chem. Soc. Rev. 2009, 38, 2046-2064.

(22) Fox, S. C.; Li, B.; Xu, D.; Edgar, K. J. Regioselective esterification and etherification of cellulose: a review. Biomacromolecules 2011, 12, 1956-1972.

(23) Liebert, T.; Hänsch, C.; Heinze, T. Click Chemistry with Polysaccharides. Macromol. Rapid Commun. 2006, 27, 208-213.

(24) Pierre-Antoine, F.; François, B.; Rachida, Z. Crosslinked cellulose developed by CuAAC, a route to new materials. Carbohydr. Res. 2012, 356, 247-251. 
(25) Koschella, A.; Chien, C.-Y.; Iwata, T.; Thonhofer, M. S.; Wrodnigg, T. M.; Heinze, T. All Sugar Based Cellulose Derivatives Synthesized by Azide-Alkyne Click Chemistry. Macromol. Chem. Phys. 2019, 19, 1900343

(26) Rahn, K.; Diamantoglou, M.; Klemm, D.; Berghmans, H.; Heinze, T. Homogeneous synthesis of cellulose p-toluenesulfonates in N,N-dimethylacetamide/LiCl solvent system. Angew. Makromol. Chemie 1996, 238, 143-163.

(27) Fox, S. C.; Edgar, K. J. Synthesis of regioselectively brominated cellulose esters and 6-cyano-6deoxycellulose esters. Cellulose 2011, 18, 1305-1314.

(28) Matsui, Y.; Ishikawa, J.; Kamitakahara, H.; Takano, T.; Nakatsubo, F. Facile synthesis of 6-amino6-deoxycellulose. Carbohydr. Res. 2005, 340, 1403-1406.

(29) Furuhata, K.-i.; Koganei, K.; Chang, H.-S.; Aoki, N.; Sakamoto, M. Dissolution of cellulose in lithium bromide-organic solvent systems and homogeneous bromination of cellulose with $\mathrm{N}$ bromosuccinimide-triphenylphosphine in lithium bromide- $\mathrm{N}, \mathrm{N}$-dimethylacetamide. Carbohydr. Res. $1992,230,165-177$.

(30) Koschella, A.; Hartlieb, M.; Heinze, T. A "click-chemistry" approach to cellulose-based hydrogels. Carbohydr. Polym. 2011, 86, 154-161.

(31) Peng, P.; Cao, X.; Peng, F.; Bian, J.; Xu, F.; Sun, R. Binding cellulose and chitosan via click chemistry: Synthesis, characterization, and formation of some hollow tubes. J. Polym. Sci. A Polym. Chem. 2012, $50,5201-5210$

(32) Vega, B.; Wondraczek, H.; Bretschneider, L.; Näreoja, T.; Fardim, P.; Heinze, T. Preparation of reactive fibre interfaces using multifunctional cellulose derivatives. Carbohydr. Polym. 2015, 132, 261273.

(33) Nagai, A.; Miller, J. B.; Kos, P.; Elkassih, S.; Xiong, H.; Siegwart, D. J. Tumor Imaging Based on Photon Upconversion of Pt(II) Porphyrin Rhodamine Co-modified NIR Excitable Cellulose Enhanced by Aggregation. ACS Biomater. Sci. Eng. 2015, 1, 1206-1210.

(34) Cao, L.; Wang, Y. Alkyne cellulose for Huisgen [3 + 2] cycloaddition with azido-terminated targets. J. Appl. Polym. Sci. 2017, 134, 25. 
(35) Zineb, K.; Kerim, N. T. K. J.; Claire, B.; Tan-Sothéa, O.; Amel, H.-B.; Rachida, Z. Synthesis, Characterization and Biological Evaluation of Triclosan Grafted onto Cellulosic Fibers. Fibers Polym. 2018, 19, 1820-1827.

(36) Gray, C. J.; Migas, L. G.; Barran, P. E.; Pagel, K.; Seeberger, P. H.; Eyers, C. E.; Boons, G.-J.; Pohl, N. L. B.; Compagnon, I.; Widmalm, G.; et al. Advancing Solutions to the Carbohydrate Sequencing Challenge. J. Am. Chem. Soc. 2019, 141, 14463-14479.

(37) Su, L.; Zhao, Y.; Chen, G.; Jiang, M. Polymeric vesicles mimicking glycocalyx (PV-Gx) for studying carbohydrate-protein interactions in solution. Polym. Chem. 2012, 3, 1560.

(38) Pathigoolla, A.; Sureshan, K. M. Reverse-CD mimics with flexible linkages offer adaptable cavity sizes for guest encapsulation. Chem. Commun. 2014, 50, 317-319.

(39) Pedersen, C. M.; Olsen, J.; Brka, A. B.; Bols, M. Quantifying the electronic effects of carbohydrate hydroxy groups by using aminosugar models. Chemistry 2011, 17, 7080-7086.

(40) Shiao, T. C.; Rej, R.; Rose, M.; Pavan, G. M.; Roy, R. Synthesis of Dense and Chiral Dendritic Polyols Using Glyconanosynthon Scaffolds. Molecules 2016, 21, 448.

(41) Bodine, K. D.; Gin, D. Y.; Gin, M. S. Synthesis of readily modifiable cyclodextrin analogues via cyclodimerization of an alkynyl-azido trisaccharide. J. Am. Chem. Soc. 2004, 126, 1638-1639.

(42) Bodine, K. D.; Gin, D. Y.; Gin, M. S. Highly convergent synthesis of C3- or C2-symmetric carbohydrate macrocycles. Org. Lett. 2005, 7, 4479-4482.

(43) Pietrzik, N.; Schmollinger, D.; Ziegler, T. Dimerization of propargyl and homopropargyl 6-azido-6deoxy-glycosides upon 1,3-dipolar cycloaddition. Beilstein J. Org. Chem. 2008, 4, 30.

(44) Campo, V. L.; Carvalho, I.; Da Silva, C. H. T. P.; Schenkman, S.; Hill, L.; Nepogodiev, S. A.; Field, R. A. Cyclooligomerisation of azido-alkyne-functionalised sugars: Synthesis of 1,6-linked cyclic pseudogalactooligosaccharides and assessment of their sialylation by Trypanosoma cruzi trans-sialidase. Chem. Sci. 2010, 1, 507.

(45) Pathigoolla, A.; Gonnade, R. G.; Sureshan, K. M. Topochemical click reaction: spontaneous selfstitching of a monosaccharide to linear oligomers through lattice-controlled azide-alkyne cycloaddition. Angew. Chem., Int. Ed. 2012, 51, 4362-4366. 
(46) Lo Conte, M.; Marra, A.; Chambery, A.; Gurcha, S. S.; Besra, G. S.; Dondoni, A. Modular approach to triazole-linked 1,6- $\alpha$-D-oligomannosides to the discovery of inhibitors of Mycobacterium tuberculosis cell wall synthetase. J. Org. Chem. 2010, 75, 6326-6336.

(47) Schmidt, M. S.; Götz, K. H.; Koch, W.; Grimm, T.; Ringwald, M. Studies toward the synthesis of linear triazole linked pseudo oligosaccharides and the use of ferrocene as analytical probe. Carbohydr. Res. 2016, 425, 28-34.

(48) Richter, A.; Klemm, D. Regioselective sulfation of trimethylsilyl cellulose using different SO3 complexes. Cellulose 2003, 10, 133-138.

(49) Mormann, W.; Wezstein, M. Trimethylsilylation of cellulose in ionic liquids. Macromol. Biosci. $2009,9,369-375$.

(50) Hassner, A.; Stern, M. Synthesis of Alkyl Azides with a Polymeric Reagent. Angew. Chem., Int. Ed. $1986,25,478-479$.

(51) Kostag, M.; Köhler, S.; Liebert, T.; Heinze, T. Pure Cellulose Nanoparticles from Trimethylsilyl Cellulose. Macromol. Symp. 2010, 294, 96-106.

(52) Hein, J. E.; Fokin, V. V. Copper-catalyzed azide-alkyne cycloaddition (CuAAC) and beyond: New reactivity of copper(I) acetylides. Chem. Soc. Rev. 2010, 39, 1302-1315.

(53) Zhang, S.; Maidenberg, Y.; Luo, K.; Koberstein, J. T. Adjusting the surface areal density of clickreactive azide groups by kinetic control of the azide substitution reaction on bromine-functional SAMs. Langmuir 2014, 30, 6071-6078.

(54) Böttcher, H.; Hallensleben, M. L.; Nuß, S.; Wurm, H. ATRP grafting from silica surface to create first and second generation of grafts. Polym. Bull. 2000, 44, 223-229.

(55) Prucker, O.; Rühe, J. Synthesis of Poly(styrene) Monolayers Attached to High Surface Area Silica Gels through Self-Assembled Monolayers of Azo Initiators. Macromolecules 1998, 31, 592-601.

(56) Ikuta, D.; Hirata, Y.; Wakamori, S.; Shimada, H.; Tomabechi, Y.; Kawasaki, Y.; Ikeuchi, K.; Hagimori, T.; Matsumoto, S.; Yamada, H. Conformationally supple glucose monomers enable synthesis of the smallest cyclodextrins. Science 2019, 364, 674. 
(57) Pathigoolla, A.; Sureshan, K. M. A crystal-to-crystal synthesis of triazolyl-linked polysaccharide. Angew. Chem., Int. Ed. 2013, 52, 8671-8675.

(58) Pathigoolla, A.; Sureshan, K. M. The topochemical synthesis of triazole-linked homobasic DNA. Chem. Commun. 2016, 52, 886-888.

(59) Su, L.; Khan, S.; Fan, J.; Lin, Y.-N.; Wang, H.; Gustafson, T. P.; Zhang, F.; Wooley, K. L. Functional sugar-based polymers and nanostructures comprised of degradable poly(d -glucose carbonate)s. Polym. Chem. 2017, 8, 1699-1707.

(60) Lin, Y.-N.; Su, L.; Smolen, J.; Li, R.; Song, Y.; Wang, H.; Dong, M.; Wooley, K. L. Co-assembly of sugar-based amphiphilic block polymers to achieve nanoparticles with tunable morphology, size, surface charge, and acid-responsive behavior. Mater. Chem. Front. 2018, 2, 2230-2238. 


\title{
Manuskript 1
}

\section{Efficient synthesis of organosoluble 6-azido-6-deoxy-2,3-0-trimethylsilyl cellulose for click reactions}

\author{
Pascal Fuchs ${ }^{\dagger}$ and Kai Zhang ${ }^{+*}$ \\ + Wood Technology and Wood Chemistry, University of Goettingen, Buesgenweg 4, 37077 Goettingen, \\ Germany \\ * Corresponding author. E-mail: kai.zhang@uni-goettingen.de
}

Originally published in:

Carbohydrate Polymers

Elsevier Ltd.

DOI: 10.1016/j.carbpol.2018.11.003

Received 4 July 2018; Received in revised form 30 October 2018; Accepted 2 November 2018 


\section{Abstract}

Huisgen cycloaddition has proven to be a powerful tool for the derivatization of cellulose, but the choice of reaction partners is limited by the solubility of the cellulose derivative. In this report, we show the efficient synthesis of an azide-bearing, organosoluble cellulose derivative via the 6-bromo-6deoxy-2,3-0-trimethylsilyl cellulose (Br-TMSC) obtained through a novel one-pot reaction. It opens the possibility to perform the copper(I)-catalyzed azide-alkyne cycloaddition (CuAAC) under moisture free conditions. First, the one-pot approach was used to synthesize Br-TMSC by combining homogeneous and regioselective bromination at C-6 position and silylation at C-2/C-3 positions. Then, 6-azido-6deoxy-2,3-0-trimethylsilyl cellulose ( $\mathrm{N}_{3}$-TMSC) as the starting material for the CuAAC was obtained via nucleophilic substitution of the bromine by azide moiety. Furthermore, $\mathrm{N}_{3}$-TMSC was exemplarily reacted with phenylacetylene and 3-cyclopentyl-1-propyne in the CUAAC in dry tetrahydrofuran (THF) as solvent and showed nearly complete conversion of the azide functionality. All products have been characterized by FTIR spectroscopy, NMR spectroscopy and elemental analysis.

\section{Introduction}

Cellulose is readily accessible to diverse chemical functionalizations, which demonstrate an important route to structure and property design.[1,2] Among others, esterification and etherification reactions are mostly used chemical modifications for the incorporation of diverse functional moieties.[3-8] However, only limited types of functional groups can be directly introduced in cellulose backbone via these synthetic routes.

In order to introduce moieties that are not directly accessible, more synthetic processes have to be used, e.g. click reactions under mild reactions. [9] Click chemistry was described by Sharpless et al. and has been used in widely spread research fields since then.[10] Click reactions run under mild conditions and significantly broaden the scope of functionalization. One of the most often used click reactions is the azide-alkyne Huisgen cycloaddition.[11] To utilize this reaction for the derivatization of cellulose, it needs to contain azide or alkyne moieties along its backbone.[12,13] A two-step reaction sequence is generally employed to introduce azide groups into the cellulose chain. It contains the introduction of a leaving group in the first step that can be substituted by the desired azide moieties during the subsequent step.

6-Tosylcellulose and 6-bromo-6-deoxy cellulose are often used intermediates for this purpose.[14-16] Comparing these two cellulose derivatives, the main advantage of the tosylated cellulose with a degree of substitution (DS) of 1 is its good solubility in $N, N$-dimethylformamide (DMF), dimethylsulfoxide (DMSO) or $\mathrm{N}, \mathrm{N}$-dimethylacetamide (DMAc), which allows an easy nucleophilic substitution under 
homogeneous reaction conditions.[16] The tosylation occurs preferably at C-6, although C-2 and C-3 can be esterified to a minor extent too. $[9,13]$ In comparison, a more regular product is obtained by using $N$-bromosuccinimide and triphenylphosphine for the bromination.[17] The bromination mechanism requires $\mathrm{S}_{\mathrm{N}} 2$ substitution of the oxyphosphonium intermediate. This is energetically disfavored at secondary hydroxyl groups. Therefore, only primary hydroxyl groups can be brominated, leading to the homogeneous and regioselective bromination of C-6 position and an adjustable DS of maximal 1.[18] However, 6-bromo-6-deoxy cellulose generally shows poor solubility and can only be swollen in common solvents, such as DMSO and DMF.[17] To overcome the drawback of the poor solubility, brominated cellulose can be further modified through esterification with acetyl groups. $[19,20]$ This improves the solubility of derivatives in organic solvents, but decreases the scope of future reactions by blocking all hydroxyl groups for consecutive reaction steps. Compared to many other functional groups, the trimethylsilyl (TMS) groups at cellulose backbone are easily hydrolysable.[21-23] Moreover, most synthesis methods for such cellulose derivatives contain a few synthesis steps, which are generally complicated and less efficient with non-uniform molecular skeletons.

Thus, in this work, regioselectively substituted 6-bromo-6-deoxy cellulose with improved solubility was synthesized via a novel efficient one-pot approach by protecting the secondary hydroxyl groups with trimethylsilyl groups. With these cellulose derivatives, the copper-catalyzed azid-alkyne cycloaddition (CUAAC) were readily performed in water-free organic solvents. Various groups, such as phenyl groups and cyclopentyl groups, were efficiently and regioselectively grafted onto cellulose backbones via CUAAC.

\section{Experimental}

\section{Materials}

Microcrystalline cellulose Avicel PH-101 (MCC) from Sigma Aldrich was dried at $103^{\circ} \mathrm{C}$ overnight prior use. THF from Bernd Kraft GmbH was dried over sodium/benzophenone and distilled prior to use. DMAc from Carl Roth GmbH was dried over calcium hydride and freshly distilled prior to use. DMSO was dried and stored over 4 Å molecular sieves. $N$-bromosuccinimide (NBS) from Alfa Aesar GmbH was recrystallized from boiling water and dried over phosphorus pentoxide. Chlorotrimethylsilane (TMSCl) and copper(I)-iodide from Sigma Aldrich, hexamethyldisilazane (HMDS) and phenylacetylene from J\&K Scientific Ltd, 3-cyclopentyl-1-propyne from Alfa Aesar $\mathrm{GmbH}$, methanol from Th. Geyer GmbH, $\mathrm{N}, \mathrm{N}$-diisopropylethylamine (DIPEA), tetrabutylammonium iodide (TBAI), triphenylphosphine $\left(\mathrm{PPh}_{3}\right)$ 
and dialysis membranes with the molecular weight cut-off of $3500 \mathrm{Da}$ from Carl Roth $\mathrm{GmbH}$ as well as sodium azide from AppliChem $\mathrm{GmbH}$ were used as received.

\section{Characterization}

${ }^{1} \mathrm{H}-\mathrm{NMR}$ spectra were obtained on a Bruker $300 \mathrm{MHz}$ NMR spectrometer, a Bruker Avance III $500 \mathrm{MHz}$ spectrometer or a Varian Inova $600 \mathrm{MHz}$ spectrometer in $\mathrm{CDCl}_{3}$ or THF-d8 at $25{ }^{\circ} \mathrm{C} .128$ scans were accumulated. DS values ascribed to TMS moieties were calculated from the ${ }^{1} \mathrm{H}-\mathrm{NMR}$ spectroscopy according to the following equation:

$$
\mathrm{DS}_{\mathrm{TMS}}=\frac{7 I_{\mathrm{TMS}}}{9 I_{\text {cellulose backbone }}}
$$

Liquid-state ${ }^{13} \mathrm{C}-\mathrm{NMR}$ and two dimensional NMR $\left({ }^{1} \mathrm{H},{ }^{1} \mathrm{H}-\mathrm{COSY}\right.$ and HSQC) spectra of all polymeric samples were obtained at $25{ }^{\circ} \mathrm{C}$ on Bruker Avance III 500 spectrometer. Scans of up to 16400 were accumulated for the ${ }^{13} \mathrm{C}-\mathrm{NMR}$ spectra. Chemical shifts are reported relative to the solvent peak.

FTIR spectra were recorded on Alpha FTIR Spectrometer (Bruker, Germany) at rt. All samples were measured between 4000 and $500 \mathrm{~cm}^{-1}$ with a resolution of $4 \mathrm{~cm}^{-1}$ using Platinum ATR and accumulated 24 scans.

The contents of carbon, hydrogen and nitrogen were determined with an Elemental Analyser 4.1 vario EL III (Elementar, Germany).

Gel permeation chromatography (GPC) characterization of all samples was performed on an Agilent 1260 Infinity system with THF as the eluent. The system was composed of an autosampler (Agilent 1260 Infinity Standard Autosampler), an isocratic solvent pump (Agilent 1260 Infinity), a precolumn of the type SDV $(8 \mathrm{~mm} \times 50 \mathrm{~mm}$, particle size $10 \mu \mathrm{m})$ from the company Polymer Standard Services (PSS), three separation columns of the type SDV $(8 \mathrm{~mm} \times 300 \mathrm{~mm}$, nominal particle size: $10 \mu \mathrm{m}$, with pore sizes of $10^{6}, 10^{5}$, and $10^{3} \AA$ ) from PSS maintained at $35^{\circ} \mathrm{C}$ in a column compartment and a refractive index detector. The flow rate of the mobile phase was $1 \mathrm{~mL} \mathrm{~min}{ }^{-1}$. The whole setup was calibrated with polystyrene standards of low dispersity and toluene as internal standard. All samples were filtered through a PTFE syringe filter with the pore size of $0.45 \mu \mathrm{m}$ before the injection. 


\section{Synthesis of cellulose derivatives}

\section{6-Bromo-6-deoxy-2,3-0-trimethylsilyl cellulose (Br-TMSC) via one-pot synthesis}

Dried MCC (3 g, $18.5 \mathrm{mmol}$ anhydroglucose units, AGUs) and $180 \mathrm{~mL} \mathrm{DMAc}$ were heated to $130^{\circ} \mathrm{C}$ and stirred for $3 \mathrm{~h}$ at this temperature. After cooling down the slurry to $100^{\circ} \mathrm{C}$, lithium bromide $(42 \mathrm{~g}$, $184 \mathrm{mmol}$ ) was added to the round bottom flask. During further cooling down to room temperature (rt) overnight, a transparent solution was obtained. Triphenylphosphine (12.18 g, $46.4 \mathrm{mmol}, 2.5 \mathrm{eq}$.) in $60 \mathrm{~mL}$ DMAc and NBS (8.28 g, $46.4 \mathrm{mmol}, 2.5$ eq.) in $60 \mathrm{~mL}$ DMAc were added drop by drop to the cellulose solution. The reaction solution was heated to $70^{\circ} \mathrm{C}$ and the reaction was allowed to continue for $2 \mathrm{~h}$. HMDS (19.26 mL, $93 \mathrm{mmol}, 5$ eq.) and TMSCl ( $600 \mu \mathrm{L}, 4.7 \mathrm{mmol}, 0.25$ eq.) were added to the reaction solution and the reaction mixture was kept for $24 \mathrm{~h}$ at $70{ }^{\circ} \mathrm{C}$. The product was precipitated from the solution and filtered off. It was dissolved in THF and reprecipitated in methanol twice. Finally, the product was dried in vacuo with a yield of $5.5 \mathrm{~g}(80 \%)$.

FTIR (ATR) in $\mathrm{cm}^{-1}, \tilde{v}=2957\left(\mathrm{CH}_{3}\right), 2900(\mathrm{C}-\mathrm{H}), 1247\left(\mathrm{CH}_{3}\right), 1046$ (C-O), 833 (C-Si).

${ }^{1} \mathrm{H}-\mathrm{NMR}\left(300 \mathrm{MHz}, \mathrm{CDCl}_{3}\right.$ ) in ppm: $\delta=5.10-2.90$ (m, 7H of AGUs), $0.18-0.15\left(\mathrm{~m}, 2 \mathrm{H}\right.$ of $\left.\mathrm{Si}_{-} \mathrm{CH}_{3}\right)$.

${ }^{13} \mathrm{C}-\mathrm{NMR}\left(125.8 \mathrm{MHz}, \mathrm{CDCl}_{3}\right)$ in ppm: $\delta=102.23$ (C-1), 78.26 (C-4), 75.86, 74.90, 74.02 (C-2/C-3/C-5), $33.60\left(\mathrm{C}-6_{\mathrm{Br}}\right), 1.38$ (TMS), 1.31 (TMS).

DS ascribed to TMS groups $\left(D_{\text {TMS }}\right)=2.2 ; \mathrm{DS}$ ascribed to bromide groups $\left(\mathrm{DS}_{\mathrm{Br}}\right)=0.8$ according to NMR measurements.

\section{6-Azido-6-deoxy-2,3-O-trimethylsilyl cellulose ( $\left.\mathrm{N}_{3}-\mathrm{TMSC}\right)$}

Br-TMSC (500 mg, $1.34 \mathrm{mmol}$ repeating units) was swollen in DMSO (40 mL). Sodium azide $(650 \mathrm{mg}$, $13.4 \mathrm{mmol}, 10$ eq.) and tetrabutylammonium iodide $(37 \mathrm{mg}, 0.1$ eq.) were added, before the temperature was increased to $70^{\circ} \mathrm{C}$. After $48 \mathrm{~h}$ reaction, the slurry was allowed to cool down to $\mathrm{rt}$ and precipitated in $200 \mathrm{~mL}$ methanol. The precipitate was filtered off, repeatedly washed with methanol and dried in vacuo yielding $350 \mathrm{mg}(79 \%)$ brown solid.

FTIR (ATR) in $\mathrm{cm}^{-1}, \tilde{v}=2957\left(\mathrm{CH}_{3}\right), 2900(\mathrm{C}-\mathrm{H}), 2100\left(\mathrm{~N}_{3}\right), 1247\left(\mathrm{CH}_{3}\right), 1046(\mathrm{C}-\mathrm{O}), 833$ (C-Si).

${ }^{1} \mathrm{H}-\mathrm{NMR}\left(300 \mathrm{MHz}, \mathrm{CDCl}_{3}\right.$ ) in ppm: $\delta=4.0-3.00$ (m, 7H of AGUs), 0.17-0.12 (m, 20H of Si-CH3).

${ }^{13} \mathrm{C}-\mathrm{NMR}(125.8 \mathrm{MHz}$, THF-d8) in ppm: $\delta=104.22-103.33$ (C-1), 81.61-78.78 (C-4), 76.74-74.19 (C-2/C-

3/C-5), 51.60 (C-6 Azide $), 33.62$ (C-6 $\left.6_{B r}\right), 1.58$ (TMS), 1.28 (TMS).

Elemental analysis (found) in wt.\%: $\mathrm{C}=41.19, \mathrm{H}=6.94, \mathrm{~N}=8.70$. 
$\mathrm{DS}_{\mathrm{TMS}}=2.0 ; \mathrm{DS}$ ascribed to azide groups $\left(\mathrm{DS}_{\text {Azide }}\right)=0.69$ according to elemental analysis .

\section{Click reactions}

\section{General procedure for CuAAC}

After $\mathrm{N}_{3}$-TMSC ( $200 \mathrm{mg}, 450 \mu \mathrm{mol}$ azide groups) was dissolved in THF (15 mL), copper(I)-iodide (43 mg, $225 \mu \mathrm{mol})$ and DIPEA (1.53 mL, $9 \mathrm{mmol})$ were added to the solution. The suspension was heated to reflux for $30 \mathrm{sec}$, before it was cooled down and stirred at $35^{\circ} \mathrm{C}$ for $20 \mathrm{~min}$. The alkyne $(2.3 \mathrm{mmol})$ was added dropwise and the reaction was allowed to continue for $7 \mathrm{~d}$. After the reaction was finished, the reaction solution was diluted with $30 \mathrm{~mL}$ THF, then solids were separated by centrifugation (12 000 $\mathrm{rpm}, 30 \mathrm{~min}, 20^{\circ} \mathrm{C}$ ) and the solution containing the product was dialyzed against THF and dried in vacuo.

\section{CuAAC with 3-cyclopenty-1-propyne}

Yield: $270 \mathrm{mg}$.

FTIR (ATR) in $\mathrm{cm}^{-1}, \tilde{v}=2957\left(\mathrm{CH}_{3}\right), 2900(\mathrm{C}-\mathrm{H}), 1247\left(\mathrm{CH}_{3}\right), 1046$ (C-O), 833 (C-Si).

${ }^{1} \mathrm{H}-\mathrm{NMR}(600 \mathrm{MHz}, \mathrm{THF}-\mathrm{d} 8)$ in ppm: $\delta=5.5-3.0$ (m, 7H of AGUs), 2.70-2.50 (m, 1.4 H, $\left.\mathrm{CH}_{2}\right), 2.23(\mathrm{~m}, 1.4$ $\mathrm{H}, \mathrm{cPe}), 2.05-1.90(\mathrm{~m}, 0.7 \mathrm{H}, \mathrm{cPe}), 1.77(\mathrm{~m}, 1.4 \mathrm{H}, \mathrm{cPe}), 1.63(\mathrm{~m}, 1.4 \mathrm{H}, \mathrm{cPe}), 1.54(\mathrm{~m}, 1.4 \mathrm{H}, \mathrm{cPe}), 0.20-$ $0.19\left(\mathrm{~m}, 14 \mathrm{H}\right.$ of $\left.\mathrm{Si}-\mathrm{CH}_{3}\right)$.

Elemental analysis (found) in wt.\%: $\mathrm{C}=47.38, \mathrm{H}=7.24, \mathrm{~N}=6.50$.

$\mathrm{DS}_{\mathrm{TMS}}=1.7 ; \mathrm{DS}$ ascribed to cyclopentyl groups $\mathrm{DS}_{\mathrm{CPe}}=0.7$ according to NMR measurements.

\section{CuAAC with phenylacetylene}

Yield: 180 mg.

FTIR (ATR) in $\mathrm{cm}^{-1}, \tilde{v}=2957\left(\mathrm{CH}_{3}\right), 2900(\mathrm{C}-\mathrm{H}), 2100\left(\mathrm{~N}_{3}\right), 1247\left(\mathrm{CH}_{3}\right), 1046(\mathrm{C}-\mathrm{O}), 833$ (C-Si), 692 (phenyl).

${ }^{1} \mathrm{H}-\mathrm{NMR}(500 \mathrm{MHz}, \mathrm{THF}-\mathrm{d} 8$ ) in ppm: $\delta=8.6-7.1(\mathrm{~m}, 2.1 \mathrm{H}$ of Ar- $H$ ), 5.0-2.5 (m, 7H of AGUs), 0.21-0.20 (m, $12 \mathrm{H}$ of $\left.\mathrm{Si}-\mathrm{CH}_{3}\right)$.

Elemental analysis (found) in wt.\%: $\mathrm{C}=47.10, \mathrm{H}=6.40, \mathrm{~N}=7.12$. 
$\mathrm{DS}_{\mathrm{TMS}}=1.6 ; \mathrm{DS}_{\mathrm{Azide}}=0.27 ; \mathrm{DS}$ ascribed to phenyl groups $\mathrm{DS}_{\mathrm{Ph}}=0.42$ according to NMR measurements.

\section{Results \& discussion}

\section{Synthesis of 6-azido-6-deoxy-2,3-0-trimethylsilyl cellulose}

In order to synthesize the cellulose derivatives with regioselectively distributed bromide and TMS moieties, a novel one-pot reaction strategy was developed (Scheme 1).

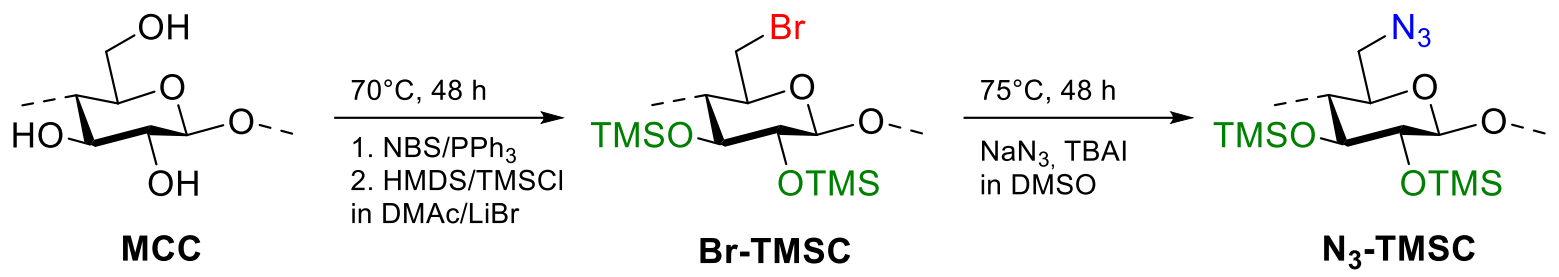

Scheme 1: Schematic illustration for the synthesis of 6-azido-6-deoxy-2,3-0-trimethylsilyl cellulose (N3-TMSC).

To ensure the uniform distribution of bromide moieties along cellulose chains, cellulose was dissolved in $\mathrm{DMAc} / \mathrm{LiBr}$ for a subsequent homogeneous modification. In the first reaction step, the hydroxyl group at C-6 is substituted with bromine by employing the $\mathrm{NBS} / \mathrm{PPh}_{3}$ (with the molar ratio of 1:1) reagent system. The molar ratio of NBS/PPh to $\mathrm{AGU}$ and the reaction time were varied, in order to find the optimal reaction condition (Table 1). $A$ DS $_{B r}$ of 0.3-0.49 was reached after one hour reaction time. In comparison, a reaction time of two hours was necessary for a higher conversion of the primary hydroxyl groups. Br-TMSC with a DS $\mathrm{Br}_{\mathrm{Br}}$ of 0.8 with a yield of $80 \%$ was obtained with 2.5 eq. of NBS/PPh . The use of 4 eq. of $\mathrm{NBS} / \mathrm{PPh}_{3}$ increased the $\mathrm{DS}_{\mathrm{Br}}$ further to 0.89 , but it was accompanied by a simultaneous decrease of the yield to $65 \%$ as well as a brownish discoloration of the product. The determination of the molecular weight via GPC shows that its $D_{n}$ was lowered to around 51 after one hour reaction time independently from the NBS/PPh 3 ratio and to around 37-39 after two hours. At the same time, $\mathrm{DP}_{\mathrm{w}}$ and therefore the $\mathrm{PDI}$ of $\mathrm{Br}-\mathrm{TMSC}$ using 2.5 eq. $\mathrm{NBS} / \mathrm{PPh}_{3}$ are higher than corresponding $\mathrm{Br}-\mathrm{TMSC}$ using 4 eq. NBS/PPh . This indicates stronger degradation of the cellulose backbone when employing 4 eq. of NBS/PPh . Therefore, the $\mathrm{Br}$-TMSC with two hours reaction time and 2.5 eq NBS/PPh 3 has been chosen for consecutive reactions.

The etherification of the remaining hydroxyl groups at C-2 and C-3 was realized by dosing HMDS and a catalytic amount of TMSCl directly to the reaction mixture without the isolation of the intermediate compounds. With longer reaction time, the product precipitated from the solution that indicates 
increasing DS ascribed to TMS groups. After one day reaction time, obtained Br-TMSC showed good solubility in chloroform or THF and can be swollen in DMSO or DMF (Table 1).

FTIR spectra of the products confirmed the successful introduction of the functional moieties (Figure 1a). The hydroxyl bands at $3330 \mathrm{~cm}^{-1}$ disappeared, which indicates the complete conversion of all hydroxyl groups. New strong peaks at 1247 and $833 \mathrm{~cm}^{-1}$ are attributed to symmetrical C-H deformation vibrations and C-Si stretching vibrations in the TMS groups. The presence of bromine at C-6 and TMS moieties within Br-TMSC was further proven by ${ }^{13} \mathrm{C}$ APT-NMR spectroscopy (Figure 1b). A signal of a carbon atom with two attached protons at $33.6 \mathrm{ppm}$ can be assigned to C-6.[19] The signal of $\mathrm{C}-1$ can be found at $102.2 \mathrm{ppm}$, the signal of $\mathrm{C}-4$ at 78.3 and the signals of $\mathrm{C}-2 / \mathrm{C}-3 / \mathrm{C}-5$ at $75.8 / 74.9 / 74$ ppm, respectively. Two strong signals of the TMS groups are located at 1.38 and $1.31 \mathrm{ppm}$.

Table 1: Overview for the synthesis and properties of all compounds as well as for the optimization of the synthesis of Br-TMSC.

\begin{tabular}{|c|c|c|c|c|c|c|c|c|}
\hline & & $\mathrm{Br}-\mathrm{TMS}$ & & & & $\mathrm{N}_{3}-\mathrm{TMSC}$ & Ph-TMSC & cPe-TMSC \\
\hline React & n time & $1 \mathrm{~h}$ & $2 \mathrm{~h}$ & $1 \mathrm{~h}$ & $2 \mathrm{~h}$ & & & \\
\hline [NBS & $\left.\mathrm{Ph}_{3}\right]$ & 2.5 eq. & 2.5 eq. & 4 eq. & 4 eq. & & & \\
\hline DS & TMS & 2.70 & 2.20 & 2.51 & 2.11 & 2.0 & 1.6 & 1.7 \\
\hline & $\mathrm{Br}$ & 0.30 & 0.80 & 0.49 & 0.89 & 0.1 & 0.1 & 0.1 \\
\hline & Azide & & & & & 0.7 & 0.27 & \\
\hline & $\mathrm{Ph}$ & & & & & & 0.42 & \\
\hline & $\mathrm{cPe}$ & & & & & & & 0.7 \\
\hline Sol. ${ }^{a}$ & THF & $+^{\mathrm{a}}$ & + & + & + & + & + & + \\
\hline & $\mathrm{CHCl}_{3}$ & + & + & + & + & - & - & sw. ${ }^{a}$ \\
\hline & DMSO & sw. & sw. & sw. & sw. & sw. & sw. & sw. \\
\hline & DMF & sw. & sw. & sw. & sw. & sw. & sw. & sw. \\
\hline GPC & $\mathrm{DP}_{\mathrm{n}}$ & 50.3 & 36.9 & 51.1 & 39.4 & n.p. ${ }^{a}$ & n.p. & 17.0 \\
\hline & $\mathrm{DP}_{\mathrm{w}}$ & 176.7 & 107.5 & 130.9 & 98.2 & & & 25.8 \\
\hline & PDI & 3.51 & 2.91 & 2.56 & 2.49 & & & 1.51 \\
\hline Yield & & $79 \%$ & $80 \%$ & $71 \%$ & $65 \%$ & $79 \%$ & & \\
\hline
\end{tabular}

a Sol.: solubility. + Soluble; - Insoluble; sw.: swelling; n.p.: not possible.

In a consecutive reaction step, the bromine of Br-TMSC was substituted by azide moieties by using sodium azide and tetrabutylammonium iodide in a catalytic amount. Hereby, Br-TMSC was swollen in 
DMSO and the reaction was run under heterogeneous conditions. The resulting polymer $\mathrm{N}_{3}$-TMSC was obtained with a yield of $79 \%$. It is soluble in THF and swellable in DMF, DMSO or toluene. Within the FTIR spectrum of $\mathrm{N}_{3}$-TMSC, a strong signal at $2099 \mathrm{~cm}^{-1}$ attributed to stretching vibrations of the $\mathrm{N} \equiv \mathrm{N}$ triple bonds appeared. The strong bands from the TMS groups at 1247 and $833 \mathrm{~cm}^{-1}$ remain unchanged. Minor formation of hydroxyl groups through desilylation can be assumed by the weak signal at $3450 \mathrm{~cm}^{-1}$. DS TMS of $\mathrm{N}_{3}$-TMSC was calculated to be 2 based on the ${ }^{1} \mathrm{H}-\mathrm{NMR}$ spectrum. Compared to the starting material $\mathrm{Br}$-TMSC, the $\mathrm{DS}_{\mathrm{TMS}}$ decreased by 0.2 . In the ${ }^{13} \mathrm{C}$ NMR spectrum (Figure $1 \mathrm{~b}$ ), the TMS signals can be found at 1.6 and $1.3 \mathrm{ppm}$. Two signals ascribed to $\mathrm{C}-6$ can be observed: a major one at $51.6 \mathrm{ppm}$ originating from C-6 containing azide groups and a minor one at $33.6 \mathrm{ppm}$ attributed to remaining C-6 bearing bromine moieties. Since $\mathrm{N}_{3}$-TMSC is composed of different repeating units with distinct compositions, the signals attributed to the carbon atoms of cellulose backbone appear as multiple peaks. The signal of C-1 can be found between 104.2$103.3 \mathrm{ppm}$, the signal of $\mathrm{C}-4$ at $81.6-78.8 \mathrm{ppm}$ and the signals of $\mathrm{C}-2 / \mathrm{C}-3 / \mathrm{C}-5$ in the range of $76.7-$ $74.2 \mathrm{ppm}$. Therefore, elemental analysis was employed to determine the DS ascribed to azide moieties ( $\left.D S_{\text {Azide }}\right)$ and the $\mathrm{DS}_{\text {Azide }}$ was calculated to be 0.69 .
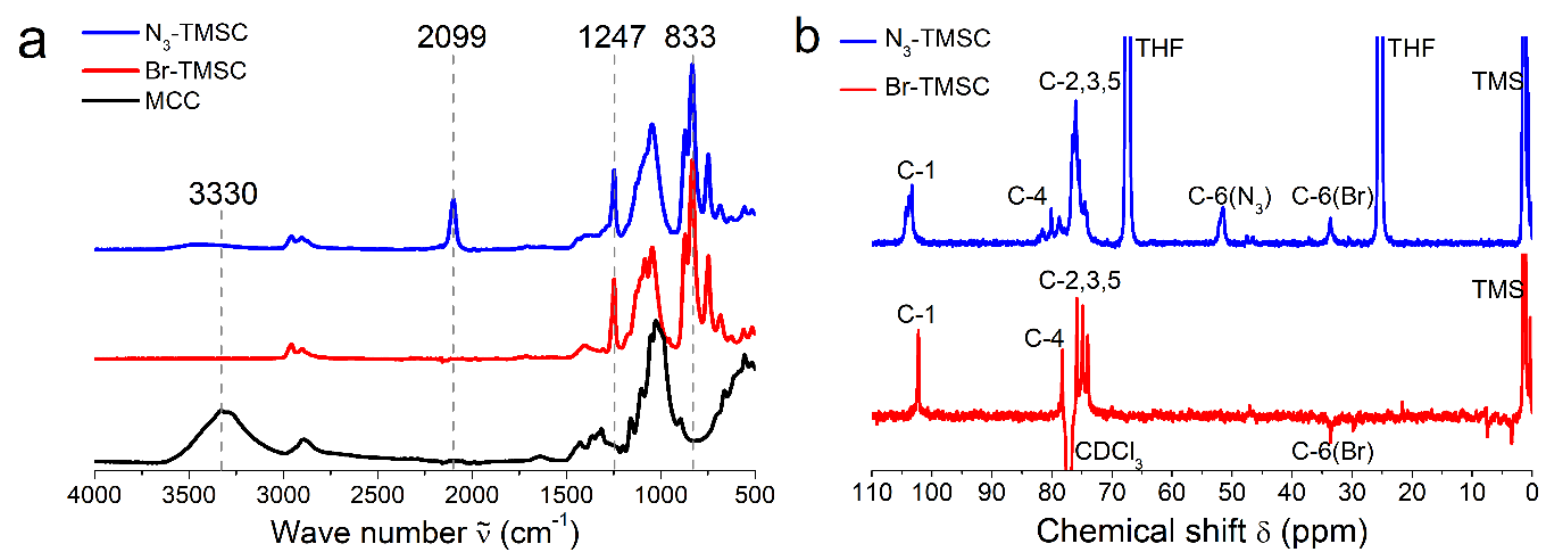

Figure 1. (a) FTIR spectra of microcrystalline cellulose (MCC), 6-bromo-6-deoxy-2,3-0-trimethylsilyl cellulose (BrTMSC) and 6-azido-6-deoxy-2,3-0-trimethylsilyl cellulose ( $\mathrm{N}_{3}$-TMSC). (b) ${ }^{13} \mathrm{C}$ APT-NMR spectrum of Br-TMSC and ${ }^{13} \mathrm{C}$ NMR spectrum of $\mathrm{N}_{3}$-TMSC.

In brief, Br-TMSC was effectively synthesized in a one-pot approach. Given that the subsequent reaction step is the nucleophilic substitution of the leaving group with azide moieties, the comparison of Br-TMSC with the commonly used 6-bromo-6-deoxy cellulose is of particular interest. Br-TMSC and 6-bromo-6-deoxy cellulose can only be swollen in DMSO. For the nucleophilic substitution of bromine with azide moieties in Br-TMSC, the protocol of Matsui et al.[17] for 6-bromo-6-deoxy cellulose was applied. The results for $\mathrm{N}_{3}$-TMSC and 6-azido-6-deoxy cellulose are similar regarding around $80 \%$ and 
nearly complete conversion of the bromine moieties, respectively. However, Br-TMSC and $\mathrm{N}_{3}$-TMSC are readily soluble in THF, compared to the corresponding cellulose derivatives without the TMS groups. Based on these results, it can be assumed that the TMS groups don't prevent or negatively affect the nucleophilic substitution reaction. Moreover, their presence along the cellulose backbone significantly improves the solubility of cellulose derivatives. A further possibility for an organosoluble cellulose derivative is the esterification of 6-bromo-6-deoxy cellulose with acetyl groups at C-2 and C-3.[20] In comparison to acetyl groups, the easier hydrolyzability of the TMS moieties can be beneficial for further reactions on the cellulose backbone. With the absence of hydroxyl groups and ester groups, a multitude of further reactions of Br-TMSC could be envisaged and pursued in future, for example the Grignard reaction, different palladium-catalyzed coupling reactions, the atom transfer radical polymerization or water-free click reactions. Such cellulose derivatives will find great potential for a broad spectrum of applications. In this report, we further show nucleophilic substitutions and further use of the organosoluble $\mathrm{N}_{3}$-TMSC as starting material for the water-free CuAAC.

\section{Huisgen-Cycloaddition}

CUAAC was performed with $\mathrm{N}_{3}$-TMSC as azide component and phenylacetylene or 3-cyclopentyl-1propyne as alkyne component (Scheme 2). The resulting polymers contained phenyl (Ph-TMSC) or cyclopentyl groups (cPe-TMSC) as functional groups on the triazole ring.

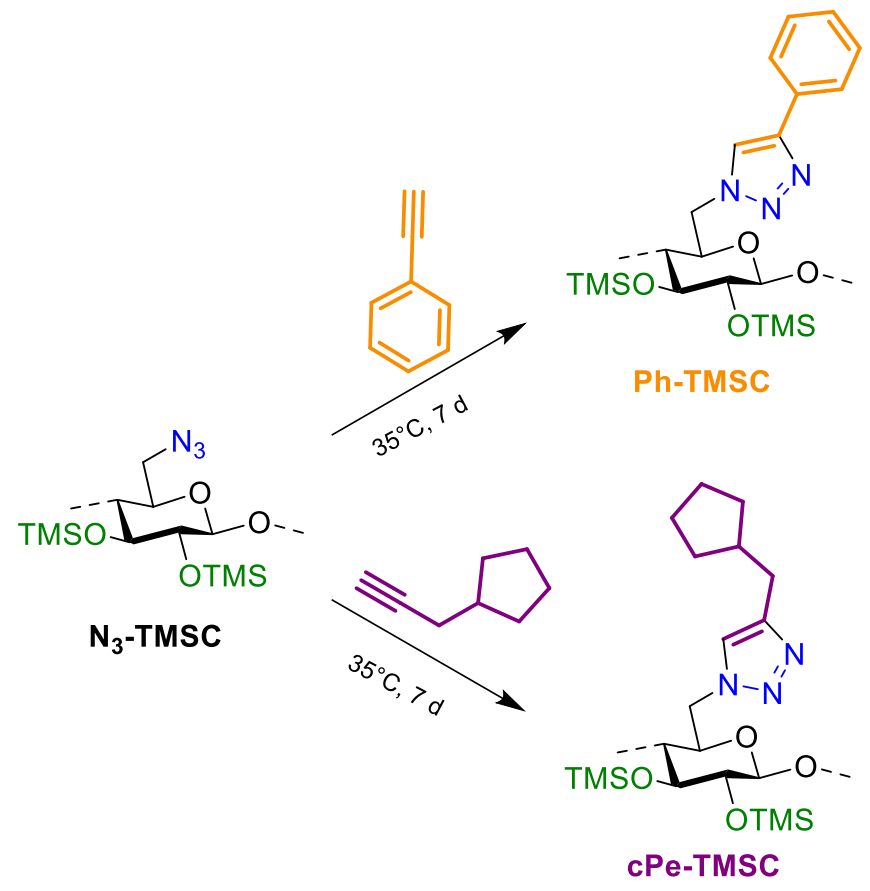

Scheme 2: Schematic illustration for CUAAC on N3-TMSC with phenylacetylene for Ph-TMSC and with 3cyclopentyl-1-propyne for cPe-TMSC. 
In FTIR spectra, the signals attributed to the azide moieties at $2099 \mathrm{~cm}^{-1}$ were used to monitor the reaction progress. Within the FTIR spectrum of Ph-TMSC, a strong intensity decrease of the signal derived from the azide groups is visible (Figure 2a). Moreover, a new peak at $692 \mathrm{~cm}^{-1}$ emerges, which is attributed to out-of-plane deformation vibrations of the phenyl rings. Within the FTIR spectrum of cPe-TMSC, no signal ascribed to azide groups can be found, indicating their complete conversion into triazole. The intensities of the peaks between 2800 and $3000 \mathrm{~cm}^{-1}$ increased due to the asymmetrical and symmetrical $\mathrm{C}-\mathrm{H}$-stretching vibrations of introduced cyclopentyl moieties, respectively.
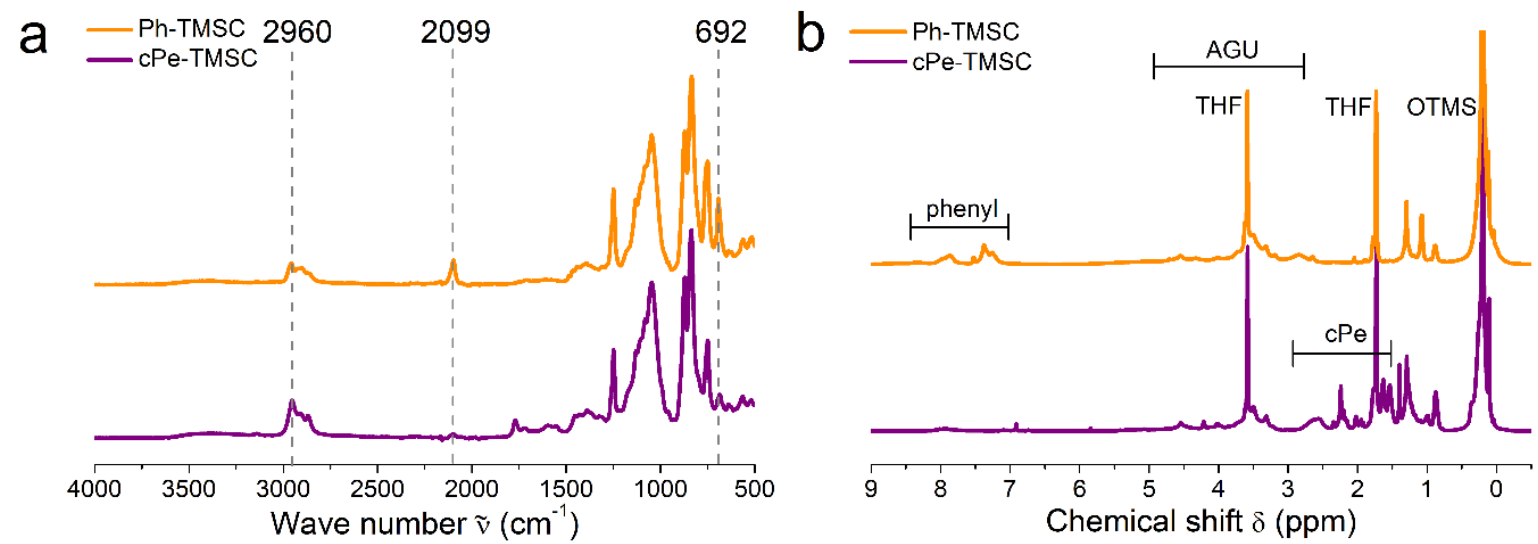

Figure 2. (a) FTIR spectra of Ph-TMSC and cPe-TMSC. (b) ${ }^{1} \mathrm{H}-\mathrm{NMR}$ spectra of Ph-TMSC and cPe-TMSC.

Within the ${ }^{1} \mathrm{H}-\mathrm{NMR}$ spectra of Ph-TMSC and cPe-TMSC (Figure 2b), the signals attributed to the AGU protons are present between 5.0-2.7 ppm and the signals derived from the TMS groups are located at $0.2 \mathrm{ppm}$. After the CUAAC reaction, the DS TMS $_{\text {Tas }}$ was generally lowered to 1.65 . Based on the integrals of signals ascribed to the aromatic protons of Ph-TMSC at 8.6 and $7.1 \mathrm{ppm}$, the $\mathrm{DS}_{\mathrm{Ph}}$ was calculated to be 0.42 under applied reaction conditions. Within the ${ }^{1} \mathrm{H}-\mathrm{NMR}$ spectrum of CPe-TMSC, the signals of the newly attached cyclopentyl groups are visible between 2.37 and $1.53 \mathrm{ppm}$. The signals derived from methylene groups are located at 2.7-2.5 ppm. Based on these ${ }^{1} \mathrm{H}-\mathrm{NMR}$ signals, $\mathrm{DS}_{\mathrm{cpe}}$ was calculated to be 0.7 . In addition, the ${ }^{1} \mathrm{H}-\mathrm{NMR}$ spectrum of Ph-TMSC shows a signal at $1.07 \mathrm{ppm}$, which should be attributed to the degraded by-products from cellulose chains, as could be proven by 2D NMR spectroscopic measurements, including ${ }^{1} \mathrm{H},{ }^{1} \mathrm{H}-\mathrm{COSY}$ and $\mathrm{HSQC}$ measurements (Figures $\mathrm{S} 1$ and S2). The signals at 1.3 and $0.9 \mathrm{ppm}$ in both spectra are attributed to silicone grease as an impurity.

In brief, we exemplarily showed the CUAAC with an aliphatic and an aromatic alkyne compound under moisture free conditions. Compared to this, the reagent system copper(II)-sulfate and sodium ascorbate in water/DMSO mixtures has been commonly used so far. $[9,13,24]$ With $\mathrm{N}_{3}$-TMSC as starting material, diverse cellulose derivatives containing distinct functional moieties can be synthesized under 
homogeneous conditions in an efficient manner, yielding full conversion of the azide functionality and little to no degradation of the cellulose backbone thanks to mild reaction conditions. On the whole, this new method not only provides a novel strategy for efficient introduction of moisture-sensitive or water-insoluble moieties onto cellulose backbone via CuAAC, but also further expands the scope of possible click reactions on cellulose and other polysaccharides.

\section{Supplementary material}

${ }^{13} \mathrm{C}-\mathrm{NMR}$ spectra of CPe-TMSC and Ph-TMSC, ${ }^{1} \mathrm{H},{ }^{1} \mathrm{H}$-COSY spectrum of Ph-TMSC and HSQC spectrum of $\mathrm{Ph}-\mathrm{TMSC}$ are to be found in Supplementary material.

\section{Acknowledgements}

P.F. and K.Z. thank German Research Foundation (DFG) for the project with the project number ZH546/3-1 for the financial support. We thank Prof. Philipp Vana and Sandra Lotze for the GPC measurements.

\section{References}

[1] T. Heinze, T. Liebert, Unconventional methods in cellulose functionalization, Progress in Polymer Science 26 (9) (2001) 1689-1762.

[2] D. Klemm, B. Heublein, H.-P. Fink, A. Bohn, Cellulose: fascinating biopolymer and sustainable raw material, Angewandte Chemie (International ed. in English) 44 (22) (2005) 3358-3393.

[3] S.C. Fox, B. Li, D. Xu, K.J. Edgar, Regioselective esterification and etherification of cellulose: a review, Biomacromolecules 12 (6) (2011) 1956-1972.

[4] T. Heinze, O.A. El Seoud, A. Koschella, Cellulose derivatives: Synthesis, structure, and properties, Springer, Cham, Switzerland, 2018.

[5] I. Cumpstey, Chemical modification of polysaccharides, ISRN organic chemistry 2013 (2013) 417672.

[6] D. Liu, K. Xia, R. Yang, Synthetic Pathways of Regioselectively Substituting Cellulose Derivatives: A Review, COC 16 (16) (2012) 1838-1849.

[7] A. Koschella, D. Fenn, N. Illy, T. Heinze, Regioselectively Functionalized Cellulose Derivatives: A Mini Review, Macromol. Symp. 244 (1) (2006) 59-73.

[8] T. Heinze (Ed.), Polysaccharides I, Springer Berlin Heidelberg, Berlin, Heidelberg, 2005. 
[9] T. Liebert, C. Hänsch, T. Heinze, Click Chemistry with Polysaccharides, Macromol. Rapid Commun. 27 (3) (2006) 208-213.

[10] H.C. Kolb, M.G. Finn, K.B. Sharpless, Click Chemistry: Diverse Chemical Function from a Few Good Reactions, Angewandte Chemie (International ed. in English) 40 (11) (2001) 2004-2021.

[11] J.E. Hein, V.V. Fokin, Copper-catalyzed azide-alkyne cycloaddition (CuAAC) and beyond: New reactivity of copper(I) acetylides, Chemical Society reviews 39 (4) (2010) 1302-1315.

[12] L. Cao, Y. Wang, Alkyne cellulose for Huisgen [3 + 2] cycloaddition with azido-terminated targets, J. Appl. Polym. Sci. 134 (4) (2017) 25.

[13] F. Pierre-Antoine, B. François, Z. Rachida, Crosslinked cellulose developed by CuAAC, a route to new materials, Carbohydrate Research 356 (2012) 247-251.

[14] T. Hasegawa, M. Umeda, M. Numata, C. Li, A.-H. Bae, T. Fujisawa, S. Haraguchi, K. Sakurai, S. Shinkai, 'Click chemistry' on polysaccharides: a convenient, general, and monitorable approach to develop (1--3)-beta-D-glucans with various functional appendages, Carbohydrate Research 341 (1) (2006) 35-40.

[15] K. Petzold-Welcke, N. Michaelis, T. Heinze, Unconventional Cellulose Products Through Nucleophilic Displacement Reactions, Macromol. Symp. 280 (1) (2009) 72-85.

[16] K. Rahn, M. Diamantoglou, D. Klemm, H. Berghmans, T. Heinze, Homogeneous synthesis of cellulose p-toluenesulfonates in $\mathrm{N}, \mathrm{N}$-dimethylacetamide/LiCl solvent system, Angew. Makromol. Chemie 238 (1) (1996) 143-163.

[17] Y. Matsui, J. Ishikawa, H. Kamitakahara, T. Takano F. Nakatsubo, Facile synthesis of 6-amino-6deoxycellulose, Carbohydrate Research 340 (7) (2005) 1403-1406.

[18] K.-i. Furuhata, K. Koganei, H.-S. Chang, N. Aoki, M. Sakamoto, Dissolution of cellulose in lithium bromide-organic solvent systems and homogeneous bromination of cellulose with $\mathrm{N}$ bromosuccinimide-triphenylphosphine in lithium bromide- $\mathrm{N}, \mathrm{N}$-dimethylacetamide, Carbohydrate Research 230 (1) (1992) 165-177.

[19] S.C. Fox, K.J. Edgar, Synthesis of regioselectively brominated cellulose esters and 6-cyano-6deoxycellulose esters, Cellulose 18 (5) (2011) 1305-1314.

[20] S. Liu, J. Liu, A.R. Esker, K.J. Edgar, An Efficient, Regioselective Pathway to Cationic and Zwitterionic N-Heterocyclic Cellulose lonomers, Biomacromolecules 17 (2) (2016) 503-513.

[21] A.P. Carapeto, A.M. Ferraria, A.M. Botelho do Rego, Trimethylsilylcellulose synthesis revisited, Polymer Testing 58 (2017) 236-240.

[22] W. Mormann, M. Wezstein, Trimethylsilylation of cellulose in ionic liquids, Macromolecular bioscience 9 (4) (2009) 369-375. 
[23] S. Köhler, T. Liebert, T. Heinze, Interactions of ionic liquids with polysaccharides. VI. Pure cellulose nanoparticles from trimethylsilyl cellulose synthesized in ionic liquids, J. Polym. Sci. A Polym. Chem. 46 (12) (2008) 4070-4080.

[24] A. Nagai, J.B. Miller, P. Kos, S. Elkassih, H. Xiong, D.J. Siegwart, Tumor Imaging Based on Photon Upconversion of Pt(II) Porphyrin Rhodamine Co-modified NIR Excitable Cellulose Enhanced by Aggregation, ACS Biomater. Sci. Eng. 1 (12) (2015) 1206-1210. 
Manuskript 1 (supporting information)

Efficient synthesis of organosoluble 6-azido-6-deoxy-2,3-0-trimethylsilyl cellulose for click reactions

Pascal Fuchs $^{\dagger}$ and Kai Zhang ${ }^{\dagger *}$ 

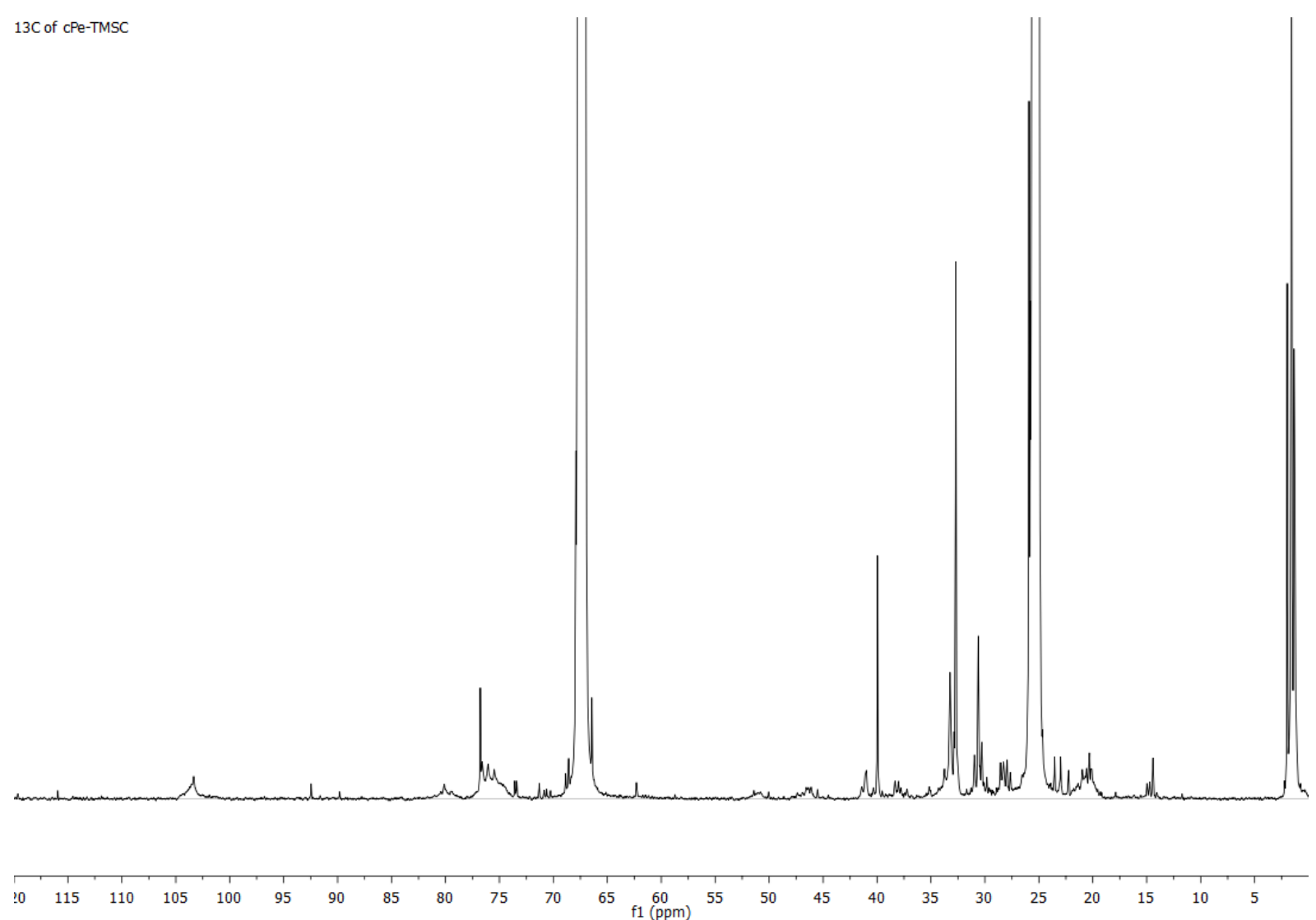

Figure S1: ${ }^{13} \mathrm{C}-\mathrm{NMR}$ spectrum of cPe-TMSC

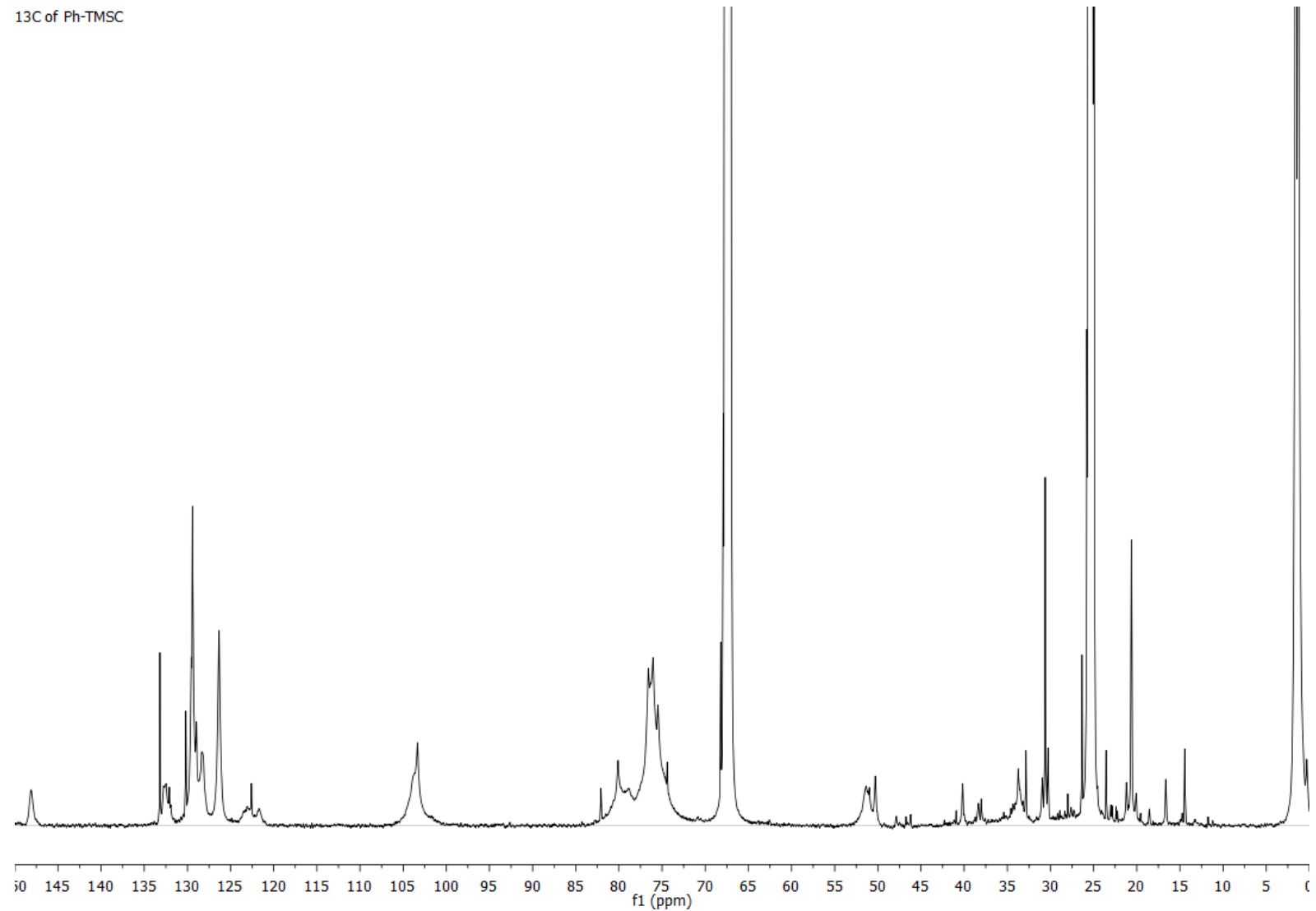

Figure S2: ${ }^{13} \mathrm{C}$-NMR spectrum of Ph-TMSC 
Manuskript 1

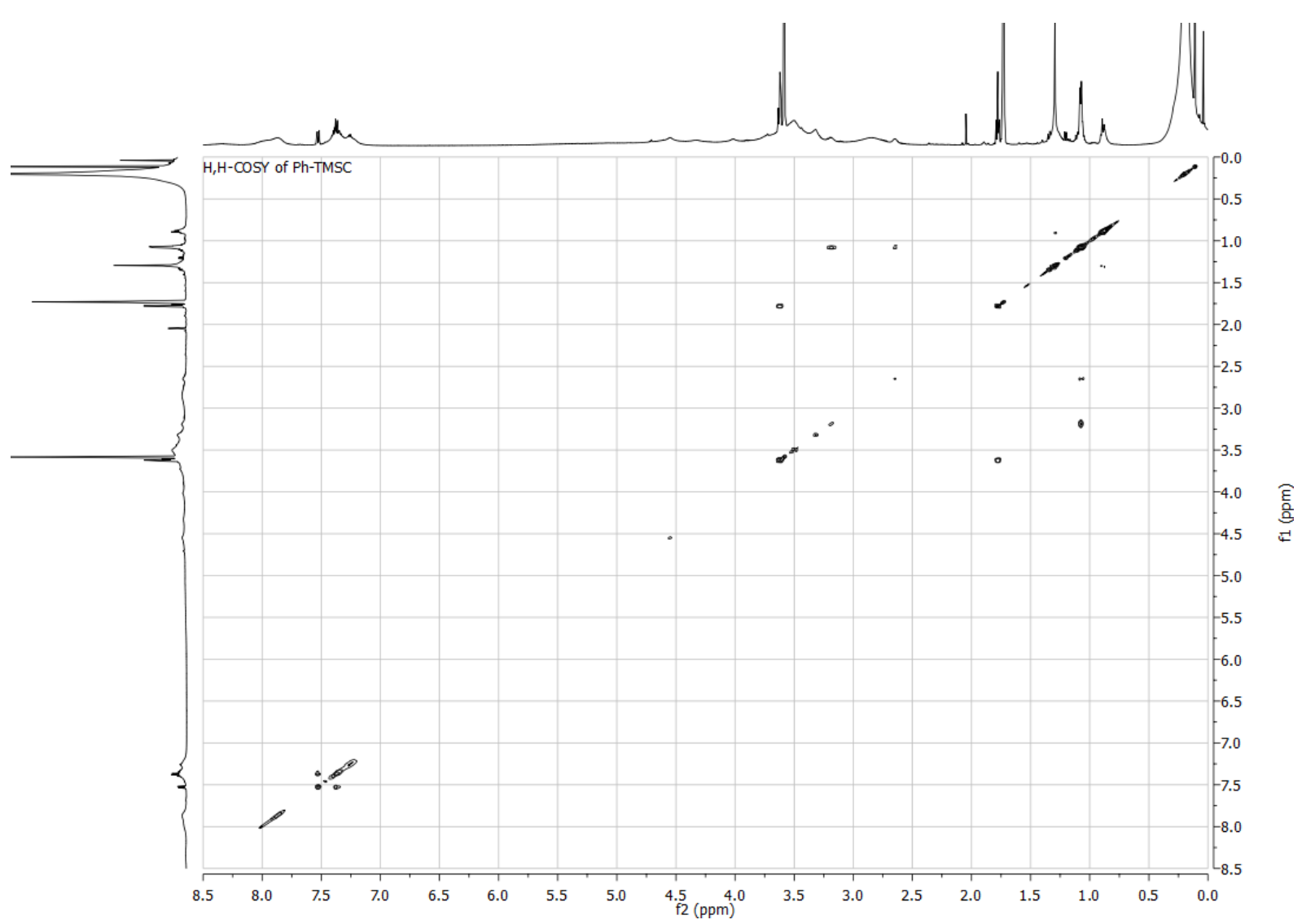

Figure S3: ${ }^{1} \mathrm{H},{ }^{1} \mathrm{H}$-COSY spectrum of Ph-TMSC.

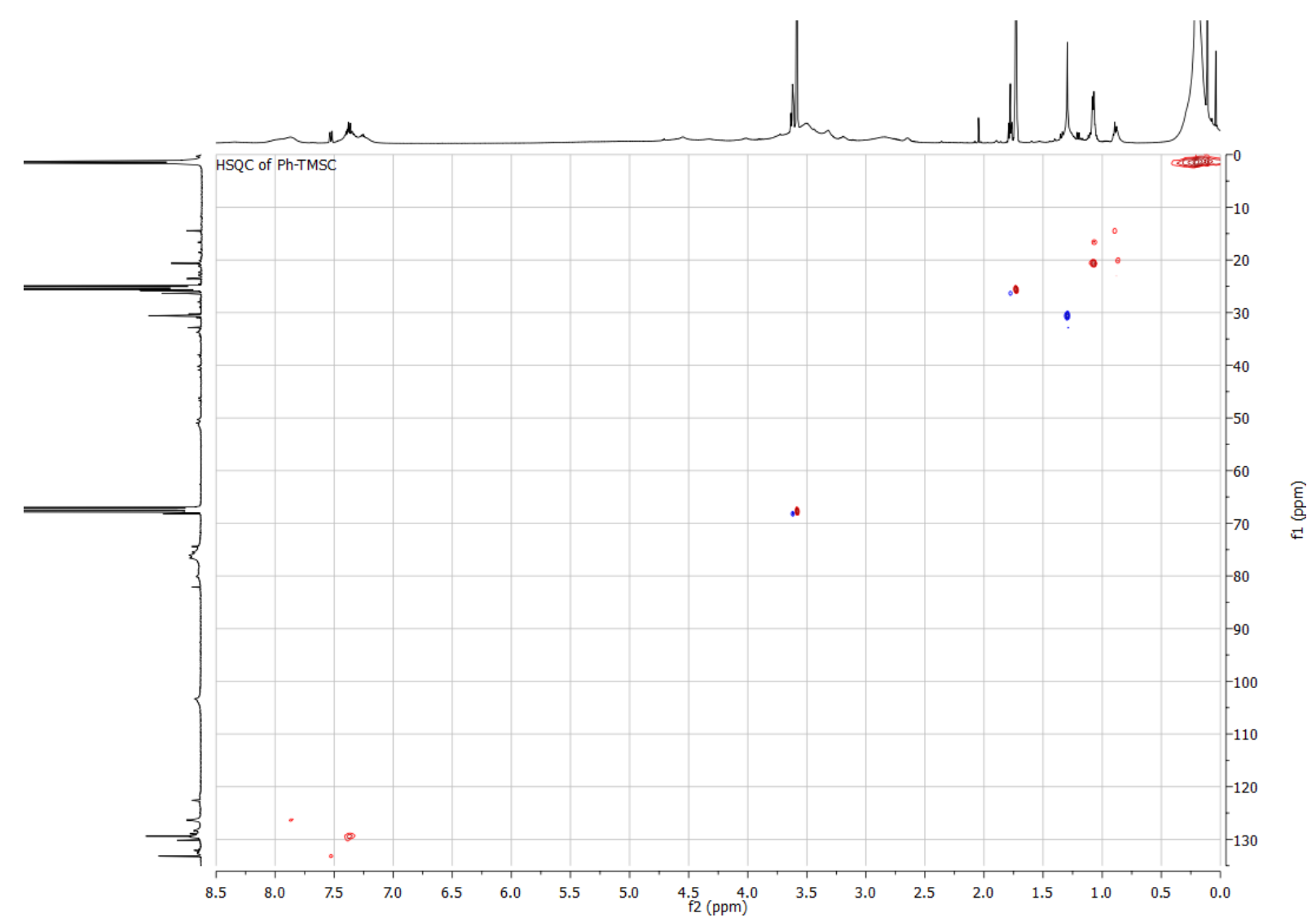

Figure S4: HSQC spectrum of Ph-TMSC.

50 


\section{Manuskript 2}

Synthesis of oligosaccharide-mimics with glucose-derived monomer via Huisgen cycloaddition through surface-initiated polymerization

Pascal Fuchs $^{\dagger}$ and Kai Zhang ${ }^{+*}$

+ Wood Technology and Wood Chemistry, University of Goettingen, Buesgenweg 4, 37077 Goettingen, Germany

* Corresponding author. E-mail: kai.zhang@uni-goettingen.de 


\section{Abstract}

The synthesis of linear and controllable oligomers and polymers using sugar-derived monomers is still challenging. Herein, we synthesized linear oligosaccharide-mimics on the surface of silica nanoparticles (SiNPs) via the polymerization of a bifunctional glucose derivative as monomer. The monomer contained an azide group at $\mathrm{C} 1$ and a propargyl group at C4 position of the glucose ring. For the successful grafting of the surface of SiNPs with the monomer, an azide-bearing chlorosilane linker was synthesized and introduced onto SiNPs surface. The Huisgen cycloaddition using the glucose-derived monomer led to the growth of linear triazole-linked oligosaccharide-mimics onto the surface of SiNPs with a degree of polymerization up to 13 and the formation of cyclic trimers and tetramers in the solution.

\section{Introduction}

Carbohydrates are a group of bioactive compounds that are structurally highly complex and serve diverse biological functions, such as cell recognition and cell binding. ${ }^{1,2}$ They play an important role in many organisms by participating in signaling events and cell-cell recognition, and they also can affect protein structure, function and stability. ${ }^{3}$ Since the purification of well-defined oligosaccharides from natural sources is often difficult or currently even impossible, chemical synthesis is an important way to provide access to them. ${ }^{4}$ Their synthesis becomes increasingly complex for oligosaccharides with more than 5-10 monosaccharide units. ${ }^{4}$ One approach to simplify the synthesis of longer oligosaccharides is to replace the glycosidic bond with triazole linkages. For this and many other applications, click chemistry found widespread use in carbohydrate chemistry in the last years. ${ }^{5-7}$

For instance, sugar backbones from different monosaccharides with introduced azide or alkyne groups were linked together to form oligosaccharides mimics. However, most studies showed the synthesis of cyclic triazole-linked oligosaccharides, ranging from dimers to heptamers. ${ }^{8-13}$

In contrast to the synthesis of cyclic oligosaccharide-mimics, the synthesis of linear oligo/polysaccharide-mimics is still challenging and less investigated. To build linear structures, the use of a starting molecule was proven useful. This starting molecule could contain an azido or an alkyne group, as well as an easily detectable group that is advantageous for further analysis. The chain growth from the starting molecule does not have a second reactive end group, which renders the cyclization impossible. For example, with ethynylferrocene as starter, the Huisgen cycloaddition of $\mathrm{N}$ acetylglucosamin-based monomer resulted in linear oligomerization up to the octamer. ${ }^{14}$ With a convergent approach, the synthesis of linear triazole-linked 1,6-oligomannosides led to linear oligomannosides up to the hexadecamer. ${ }^{15}$ Linear 1,4-triazole-linked polygalactosides were 
synthesized in a topochemical click reaction in the crystal lattice. ${ }^{16,17}$ So far, linear 1,4-triazole-linked mimics of oligosaccharides or polysaccharides based on glucose-derived monomers have not been reported yet.

Herein, we reported the synthesis of linear oligosaccharide-mimics using glucose-derived monomers. In the first step, the bifunctional glucose-derived monomer 2,3,6-tris-O-benzyl-4-O-propargyl- $\beta$-Dglucopyranosyl azide was synthesized. Furthermore, an azide-bearing chlorosilane linker was synthesized and introduced onto the surface of SiNPs, in order to have starting sites for the polymerization using the glucose-derived monomer. The surface-initiated polymerization was performed under diverse conditions for the growth of longer linear chains. After the Huisgen cycloaddition as Copper(I)-catalyzed Azide-Alkyne Cycloaddition (CUAAC) was completed, the linear oligomers were easily separated from the cyclic compounds, which were formed in the supernatant solution. Linear and cyclic products were finally characterized with diverse methods.

\section{Results and Discussion}

In order to click-polymerize glucose-derived monomer 1 on the surface of SiNPs, azide or alkyne moieties need to be present on their surface. So far, commercially available bromoalkyl silanes have been used for this modification and bromine moieties were substituted by azide moieties in a consecutive reaction step. ${ }^{18}$ However, it is generally complicated to monitor the reaction progress of the nucleophilic substitution on the surface and ensure complete conversion of bromine moieties. ${ }^{19}$ Therefore, we designed and synthesized a silane linker $\mathbf{2}$ (Scheme 1) with a terminal azide moiety. Bound to this linker, every chain grafted onto the surface of SiNPs will only contain one azide group. For the synthesis of the linker with reactive silane species, monochlorosilane was chosen over dichlorosilanes or trichlorosilanes to avoid further crosslinking or branching. Detrimentally for the use of dichlorosilanes and trichlorosilanes is their ability to oligomerize or polymerize, as soon as traces of moisture are present in the reaction system. In comparison, monochlorosilanes dimerize by reacting with water and are consequently deactivated. Hence, monochlorosilanes are advantageous for grafting individual linear chains onto the surface of SiNPs. ${ }^{20}$ In addition, the azide-bearing SiNPs- $\mathbf{N}_{\mathbf{3}}$ were well dispersed in organic media, such as THF. This excellent dispersibility assures sufficient accessibility of the azide groups for the click-catalyst as well as for the polymerization of monomer 1 . This property is attributed to the length of the non-polar linker as a spacer with 15 atoms between the surface of SiNPs and the terminal azide moieties. 


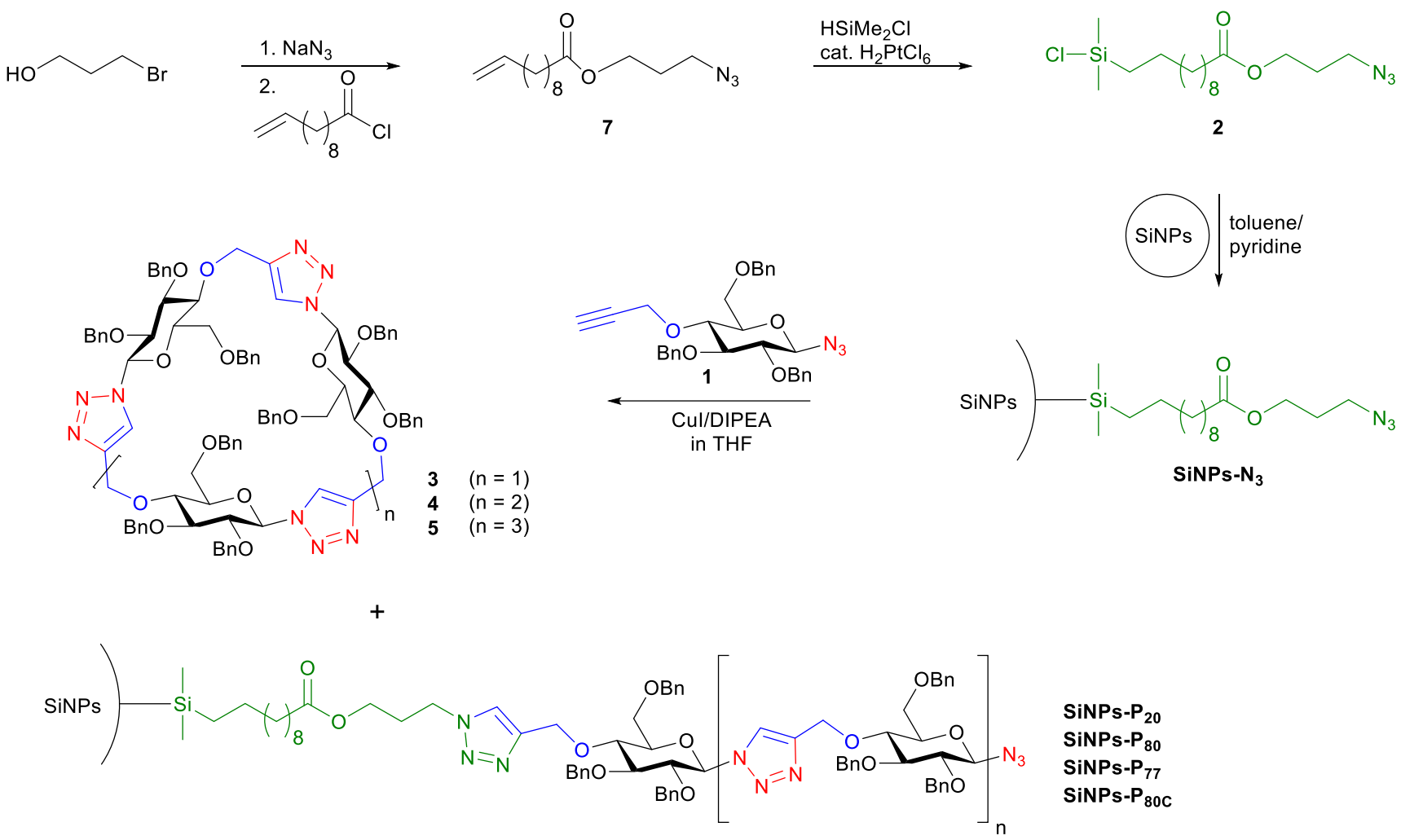

Scheme 1. Schematic illustration for the synthesis of the silane linker 2, azide-bearing silica nanoparticles SiNPs$\mathbf{N}_{\mathbf{3}}$ and subsequent Huisgen cycloaddition.

After the grafting process with $\mathbf{2}$ was finished, unreacted chlorosilane was washed off and the elemental composition was determined (Table 1). The grafting density of azide groups was calculated to be $109.5 \mu \mathrm{mol} / \mathrm{g}$ based on the nitrogen content. With the BET-surface of $49.25 \mathrm{~g} / \mathrm{m}^{2}$, the density of azide groups on the surface of SiNPs was calculated to be $2.22 \mu \mathrm{mol} / \mathrm{m}^{2}$. Compared to previous studies with shorter alkyl chains ${ }^{20,21}$, we achieved $10-20 \%$ lower grafting densities due to our longer chlorosilanes $\mathbf{2}$ with more bulky terminal groups.

Then, linear oligomer chains containing glucose-derived monomers were polymerized on the surface of SiNPs. A glucose-derived monomer 1 was designed and synthesized with high yield. ${ }^{\S}$ It is to be expected that the step-growth-type CuAAC polymerization of the monomer 1 starts with the formation of a linear dimer. This dimer bears one reactive azide and one reactive propargyl end group. As the reaction progresses further, the intermediately formed dimers could react with another monomer,

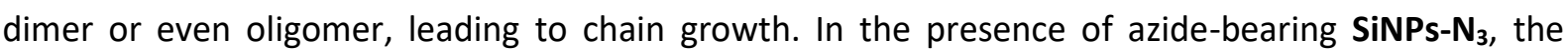
monomers, dimers or oligomers can also react with the surface-bound azide groups and form polymer chains on the surface of NPs. Thus, the reaction on the surface of SiNPs and the reaction of monomers in the solution occur parallel. The monomers of the products are connected via $\beta$-1,4-triazole linkages, 
which are different from the $\beta-1,4-$ glycosidic bonds within cellulose chains. Thus, linear products can be considered to have a pseudo-cellulosic structure.

Table 1: Elemental compositions in wt.\% of SiNPs-N $\mathbf{N}_{3}$ and SiNPs after the oligomerization of 1 under various reaction conditions, the grafting yields on the SiNPs-surface and the ratios of the peak heights of cyclic oligosaccharide-mimics $\mathbf{4 / 3}$ in the MALDI mass spectra (Figure 1a).

\begin{tabular}{|c|c|c|c|c|c|c|c|}
\hline Sample & C & $\mathrm{H}$ & $\mathrm{N}$ & DP & $\begin{array}{l}\text { Yield on } \\
\text { surface }\end{array}$ & $\begin{array}{l}\text { SiNPs-Cyclic } \\
\text { compounds }\end{array}$ & $\begin{array}{l}\text { Peak height ratio } \\
\mathbf{4 / 3}\end{array}$ \\
\hline SiNPs-N $_{3}$ & 3.50 & 1.20 & 0.46 & - & - & - & - \\
\hline SiNPs-P 20 & 13.90 & 1.86 & 1.73 & 3.46 & $17.3 \%$ & $\mathrm{C}_{20}$ & $5 \%$ \\
\hline SiNPs-P 80 & 21.30 & 2.45 & 2.57 & 6.62 & $8.3 \%$ & $\mathrm{C}_{80}$ & $19 \%$ \\
\hline SiNPs-P 77 & 24.80 & 2.70 & 2.96 & 8.44 & $11.0 \%$ & $\mathrm{C}_{77}$ & $15 \%$ \\
\hline SiNPs-P 800 & 31.75 & 3.24 & 3.73 & 13.01 & $16.3 \%$ & $\mathrm{C}_{80 \mathrm{C}}$ & 0 \\
\hline
\end{tabular}

The chain propagation continues until the reaction is terminated. One possible termination reaction is the intramolecular cyclization, in which the azide end group and propargyl end group of the same oligomer react with each other, forming a stabile triazole bond and leaving no further click-reactive group in the molecule. Hereby, cyclodextrin-analogs with the $\beta$-1,4-triazole linkage between monomers are obtained, compared to the $\alpha-1,4$-glycosidic linkage of cyclodextrins. This termination reaction is not possible for the oligomerization of monomers 1 on the surface of SiNPs- $\mathbf{N}_{\mathbf{3}}$ bearing pendant azide groups (Scheme 1), which assures the formation of linear oligomer chains on SiNPs. After the reaction, modified SiNPs were separated from the reaction solution via centrifugation.

Because the surroundings of the click reaction were already adapted, we changed the dosage of added monomer 1 and the feeding frequency of the monomer $\mathbf{1}$ to tune the lengths of linear oligomers. For the first experiments, 20 equivalents of the monomer 1 were added to 1 equivalent of azide groups on SiNPs- $\mathbf{N}_{3}$. After the reaction, resulting SiNPs-P $\mathbf{P}_{20}$ were separated from the supernatant solution by centrifugation. Oligomer chains with a calculated DP of 3.46 on the surface of SiNPs-P $\mathbf{P}_{\mathbf{2 0}}$ were obtained, according to the results of elemental analysis (Table 1). The CUAAC of the remaining 16.54 equivalents of 1 took place in the solution. MALDI mass spectroscopic analysis of the supernatant showed the presence of diverse oligomers in the mixture $\mathbf{C}_{\mathbf{2 0}}$ (Figure $1 \mathrm{a}$ ). To be precise, the mixture $\mathbf{C}_{\mathbf{2 0}}$ predominantly contained the pseudo-trisaccharide $\mathbf{3}$ and a minority of the pseudo-tetrasaccharide $\mathbf{4}$ (Scheme 1).

The asymmetrical stretching vibration of the azide group commonly gives a very strong signal at around $2100 \mathrm{~cm}^{-1}$ in FTIR spectra. In the spectrum of $\mathrm{C}_{20}$ (Figure $1 \mathrm{~b}$ ), this band cannot be observed. In addition, 
no signal ascribed to azide group as well as propargyl end group could be detected using proton NMR spectroscopy. Therefore, obtained products should be cyclized compounds.

When a higher amount of 80 equivalents monomer was employed, the content of the cyclic pseudotetrasaccharide $\mathbf{4}$ in the mixtures $\mathbf{C}_{\mathbf{8 0}}$ slightly increased, but the cyclic pseudo-trisaccharide $\mathbf{3}$ was still the major cyclic product (Figure 1a). In comparison, the linear oligomers on the surface of SiNPs-P.8 had a higher average DP of 6.62 (Table 1). Compared to the previous experiments of SiNPs-P $\mathbf{2}_{\mathbf{2 0}}$, the DP of linear oligomers increased by $91 \%$, although a fourfold amount of the monomer was employed. However, the yield of linear oligomers on the surface of SiNPs decreased from $17.3 \%$ for the SiNPs-P $\mathbf{2}_{\mathbf{2}}$ to $8.3 \%$ for SiNPs-P P $_{\mathbf{8 0}}$ (Table 1). It should be noted that the reaction surroundings including the volume of the reaction system were kept constant. This shows that the reaction rate of the cyclic compounds benefits more from the increased monomer concentration than the reaction on the surface of SiNPs. Conversely, the synthesis of linear oligosaccharide mimics on the surface of SiNPs should be favored by lower monomer concentrations. To prove this hypothesis, CuAAC with 77 equivalents of the monomer that was added in 7 portions hourly was conducted. By doing so, the average monomer concentration was reduced during each click reaction cycle.

Following this, the DP of the linear oligomer chains on the surface of SiNPs-P $\mathbf{P}_{77}$ increased to 8.44 and the yield increased to $11.0 \%$ (Table 1). In contrast, the impact on the obtained cyclic oligomers was minimal, as shown in their MALDI mass spectrum ( $\mathbf{C}_{77}$, Figure $\left.1 \mathrm{a}\right)$. Therefore, a reduced monomer concentration for each single CuAAC step during the whole synthesis will elevate the DP of linear oligomers.

By employing 80 equivalents of the monomer for a continuous addition of the monomer over a time period of 24 hours during the CUAAC reaction, the average active monomer concentration is much lower compared to the previous experiments. After the reaction, the DP of obtained linear oligomers on the surface of resulting SiNPs-P $\mathbf{P}_{\mathbf{8 0}}$ further increased to 13.01 (Table 1). Compared to the previous experiments with the one-pot addition with the same amount of the monomer (SiNPs-P80), the DP increased by $\mathbf{9 7 \%}$. The grafting yield of $16.3 \%$ is similar to the first experiment SiNPs-P $\mathbf{P}_{\mathbf{2 0}}$. Moreover, only the cyclic trimer was formed in the solution based on MALDI mass spectrum (Figure 1a). In other previous reports about a step-growth-type CuAAC of monosaccharide-derived monomers, oligomers with DPs not higher than 6-8 were often obtained. ${ }^{14,22}$ Compared to these results, we obtained significantly enhanced higher average DPs of up to 13 . In addition, the separation and purification steps of obtained products are more convenient than the chromatographic methods. 

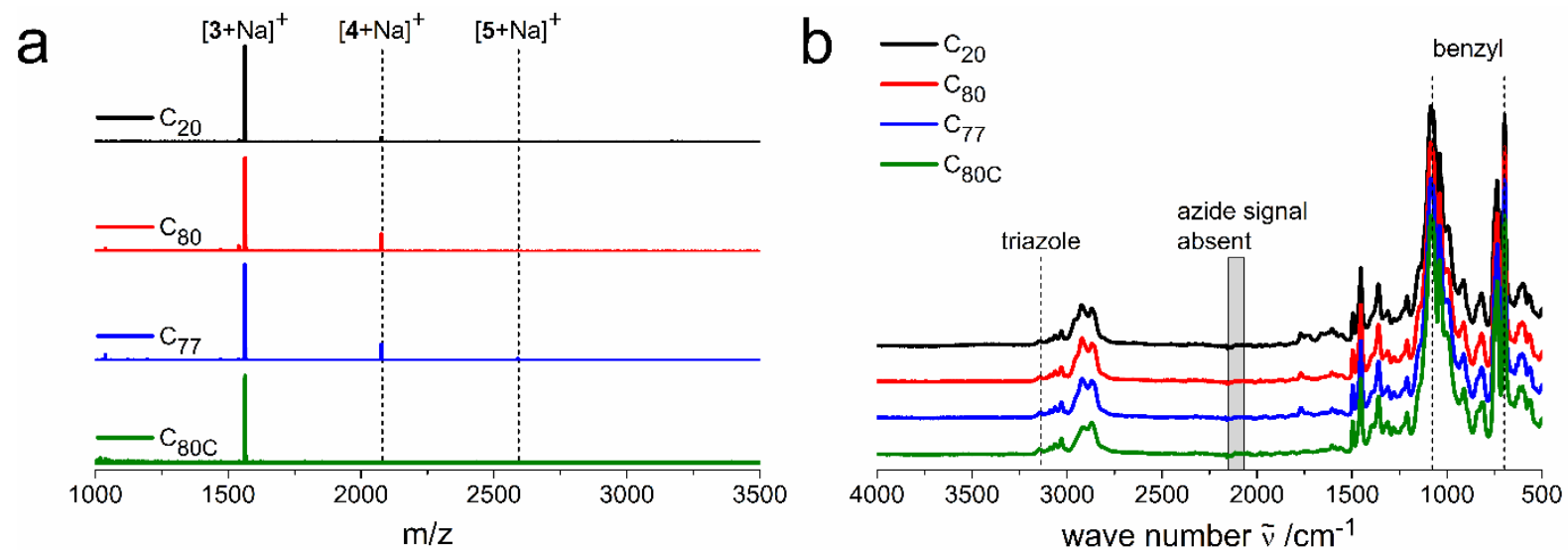

Figure 1. (a) Representative MALDI mass spectra of the cyclic oligomers from diverse experiments. The molecular masses of $\mathbf{3}$ and $\mathbf{4}$ (Scheme 1) were found with sodium ions as the counterion. (b) FTIR spectra of the cyclic oligomers from diverse experiments.

Although a higher DP of 16 was reached previously using the Huisgen cycloaddition in a more complex convergent approach, the synthetic complexity increased significantly with a higher targeted chain length. ${ }^{15}$ In our approach, chain length can be easily tuned by the amount of employed monomer and/or the feeding frequency. Even longer chains were reported to be accessible by using ring opening polymerization, e.g. the synthesis of 4,6-carbonate linked polyglucoside with a DP up to $100 .{ }^{23}$ However, such a ring opening polymerization may be difficult for the synthesis of $\beta-1,4$-carbonatelinked oligosaccharide-mimics due to the strong steric obstacle during monomer synthesis.

Cyclic dimerization was not observed in any experiment. The dimer would consist of only two pyranose rings and two rigid triazole rings with a bridging methylene group, which will have a size comparable to cyclodextrins with three to four glucopyranose rings. The synthesis of rings in this size is only possible, when the monosaccharides are locked in the right confirmation. ${ }^{24}$ Cyclic dimerization was reported for a galactose-based monomer that differs from 1 by the configuration at $\mathrm{C} 4 .{ }^{13}$ Thus, the orientation of the reactive groups at $\mathrm{C} 1$ and $\mathrm{C} 4$ and the steric hindrance by the bulky benzyl groups should be critical for the cyclic dimerization.

Based on above results, we obtained the cyclic pseudo-trisaccharide $\mathbf{3}$ as smallest cyclic compound. It was either the main or the only product in the solution after the CuAAC reaction of monomer 1 . The cyclic pseudo-tetrasaccharide $\mathbf{4}$ was formed to a minor extent, while larger cyclic compounds were not detected at all (Table 1). Moreover, the intramolecular cyclization for $\mathbf{3}$ and the chain growth by the addition of one more monomer are two competing reactions during the CUAAC oligomerization. Comparing the peak height ratios of $\mathbf{4 / 3}$ in the MALDI mass spectra (Figure $1 \mathrm{a}$ and Table 1 ), it is visible that more $\mathbf{4}$ was preferentially formed during reactions with higher monomer concentrations $\left(\mathbf{C}_{\mathbf{8 0}}\right.$ and $\left.C_{77}\right)$. 
With lower monomer concentration and therefore higher yield of pseudo-cellulose on the NPs surface, 4 was formed only to a minor extent $\left(\mathbf{C}_{\mathbf{2 0}}\right)$ or not formed at all $\left(\mathbf{C}_{\mathbf{8 0}}\right)$. Therefore, the intramolecular cyclization for the synthesis of $\mathbf{3}$ was preferred with the presence of low amounts of glucose-derived monomers, compared to further chain growth and cyclization to 4 . At the same time, the continuous chain growth of linear oligomers at the surface of SiNPs was preferred as well.

\section{Conclusions}

In brief, we showed a novel method to synthesize linear oligosaccharide-mimics using glucose-derived monomer via Huisgen cycloaddition. Compared to other synthesis pathways that rely on chromatographic methods for the product separation, this procedure has major advantages regarding the facile separation and adjustment of chain lengths, which enables the large scale synthesis of oligosaccharide mimics. In general, the procedure in this report could be applied to monomers derived from various saccharides and starters. It further expands the scope for the synthesis of diverse oligosaccharide-mimics with various functional groups and applications.

\section{Conflicts of interest}

There are no conflicts to declare.

\section{Notes and references}

$\S \quad$ Detailed reaction scheme can be found in supporting information (Scheme S1).

1 T. J. Boltje, T. Buskas and G.-J. Boons, Nat. Chem., 2009, 1, 611-622.

2 M. Delbianco, P. Bharate, S. Varela-Aramburu and P. H. Seeberger, Chem. Rev., 2016, 116, 1693-1752.

3 L. Krasnova and C.-H. Wong, J. Am. Chem. Soc., 2019, 141, 3735-3754.

$4 \quad$ O. Calin, S. Eller and P. H. Seeberger, Angew. Chem., Int. Ed., 2013, 52, 5862-5865.

$5 \quad$ Z. J. Witczak and R. Bielski, Click Chem. Glycosci., Wiley, Hoboken N.J., 2013.

6 D. Kushwaha, P. Dwivedi, S. K. Kuanar and V. K. Tiwari, Curr. Org. Synth., 2013, 10, 90-135.

7 V. K. Tiwari, B. B. Mishra, K. B. Mishra, N. Mishra, A. S. Singh and X. Chen, Chem. Rev., 2016, 116, 3086-3240. 
9 V. L. Campo, I. Carvalho, C. H. T. P. Da Silva, S. Schenkman, L. Hill, S. A. Nepogodiev and R. A. Field, Chem. Sci., 2010, 1, 507.

K. D. Bodine, D. Y. Gin and M. S. Gin, J. Am. Chem. Soc., 2004, 126, 1638-1639.

K. D. Bodine, D. Y. Gin and M. S. Gin, Org. Lett., 2005, 7, 4479-4482.

A. Pathigoolla and K. M. Sureshan, Chem. Commun., 2014, 50, 317-319.

M. S. Schmidt, K. H. Götz, W. Koch, T. Grimm and M. Ringwald, Carbohydr. Res., 2016, 425, 2834.

M. Lo Conte, A. Marra, A. Chambery, S. S. Gurcha, G. S. Besra and A. Dondoni, J. Org. Chem, $2010,75,6326-6336$.

A. Pathigoolla and K. M. Sureshan, Angew. Chem., Int. Ed., 2013, 52, 8671-8675.

A. Pathigoolla, R. G. Gonnade and K. M. Sureshan, Angew. Chem., Int. Ed., 2012, 124, 44384442.

18 T. Lummerstorfer and H. Hoffmann, J. Phys. Chem. B, 2004, 108, 3963-3966.

S. Zhang, Y. Maidenberg, K. Luo and J. T. Koberstein, Langmuir, 2014, 30, 6071-6078.

20 O. Prucker and J. Rühe, Macromolecules, 1998, 31, 592-601.

21 H. Böttcher, M. L. Hallensleben, S. Nuß and H. Wurm, Polym. Bull., 2000, 44, 223-229.

22 V. L. Campo, I. M. Ivanova, I. Carvalho, C. D. Lopes, Z. A. Carneiro, G. Saalbach, S. Schenkman, J. S. da Silva, S. A. Nepogodiev and R. A. Field, Tetrahedron, 2015, 71, 7344-7353.

23 Y.-N. Lin, L. Su, J. Smolen, R. Li, Y. Song, H. Wang, M. Dong and K. L. Wooley, Mater. Chem. Front., 2018, 2, 2230-2238.

24 D. Ikuta, Y. Hirata, S. Wakamori, H. Shimada, Y. Tomabechi, Y. Kawasaki, K. Ikeuchi, T. Hagimori, S. Matsumoto and H. Yamada, Science, 2019, 364, 674. 


\section{Manuskript 2 (Supporting information)}

\section{Synthesis of oligosaccharide-mimics with sugar-based monomer via Huisgen cycloaddition through surface-initiated polymerization}

Pascal Fuchs and Kai Zhang*

\section{Experimental Section}

\section{General}

$\mathrm{N}, \mathrm{N}$-Dimethylsulfoxide from Carl Roth $\mathrm{GmbH}, \mathrm{N}, \mathrm{N}$-dimethylformamide (DMF) and dichloromethane (DCM) from Th. Geyer Ingredients $\mathrm{GmbH}$ were dried over $4 \AA$ molecular sieves. Toluene and tetrahydrofurane (THF) from Th. Geyer Ingredients $\mathrm{GmbH}$ were dried over sodium/benzophenone and distilled prior to use. Pyridine from Abcr $\mathrm{GmbH}$, propargyl bromide and chlorodimethylsilane from Alfa aesar, diethyl ether from AppliChem $\mathrm{GmbH}$, Glucose monohydrate, hexachloroplatinic acid, iodine, acetic anhydride, chloroform, $\mathrm{N}, \mathrm{N}$-diisopropylethylamine (DIPEA) and copper(I)-iodide from Carl Roth $\mathrm{GmbH}$, hydrobromic acid (33 wt.\% in acetic acid), trifluoroacetic anhydride and trifluoroacetic acid from Honeywell, silica nanoparticles of $50 \mathrm{~nm}$ from lo-li-tec $\mathrm{GmbH}$, sodium methoxide, triethylsilane and 3-bromo-1-propanol from J\&K Scientific, sodium thiosulfate from KMF Laborchemie Handels$\mathrm{GmbH}$, triethylamine and benzyl bromide from Merck KGaA, sodium azide, $p$-toluenesulfonic acid, Dowex ${ }^{\circledR}$ 50WX8 hydrogen form 100-200 mesh, benzaldehyde dimethyl acetal, sodium hydride (60 wt.\% dispersion in mineral oil) and 10-undecenoyl chloride from Sigma-Aldrich, 2-propanol, ethanol, methanol, sodium bicarbonate, sodium sulfate, sodium chloride, $n$-hexane and ethyl acetate (EA) from Th. Geyer Ingredients $\mathrm{GmbH}$ as well as silica gel and TLC plates from VWR were used as received.

\section{Characterization}

${ }^{1} \mathrm{H}$-NMR spectra and ${ }^{1} \mathrm{H},{ }^{1} \mathrm{H}$-COSY spectra were obtained on a Bruker $300 \mathrm{MHz}$ NMR spectrometer or a Bruker Avance III $500 \mathrm{MHz}$ in $\mathrm{CDCl}_{3}$ at $25^{\circ} \mathrm{C}$. Up to 128 scans were accumulated for the ${ }^{1} \mathrm{H}-\mathrm{NMR}$ spectra. Liquid-state ${ }^{13} \mathrm{C}$-NMR and HSQC spectra of all samples were obtained at $25^{\circ} \mathrm{C}$ on Bruker Avance III 500 spectrometer. Scans of up to 2048 were accumulated for the ${ }^{13} \mathrm{C}-\mathrm{NMR}$ spectra. Chemical shifts are reported relative to the solvent peak. 
FTIR spectra were recorded on Alpha FTIR Spectrometer (Bruker, Germany) at room temperature (rt). All samples were measured between 4000 and $500 \mathrm{~cm}^{-1}$ with a resolution of $4 \mathrm{~cm}^{-1}$ using Platinum ATR and accumulated 24 scans.

The contents of carbon, hydrogen and nitrogen were determined with an Elemental Analyser 4.1 vario EL III (Elementar, Germany).

MALDI mass spectrometry was performed on an Autoflex Speed from Bruker Daltonik with a TOF detector in reflecton mode.

\section{Synthesis of glucose-derived monomer}
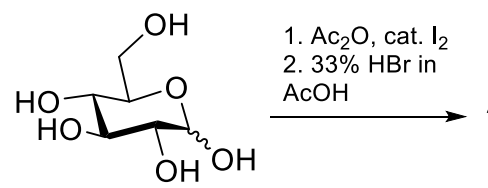$$
\begin{gathered}
\mathrm{AcO} \\
8
\end{gathered}
$$

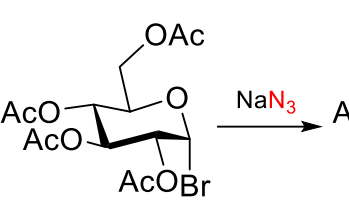

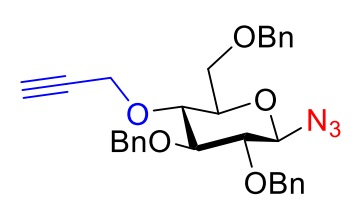

1

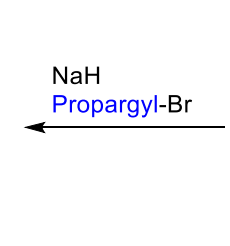

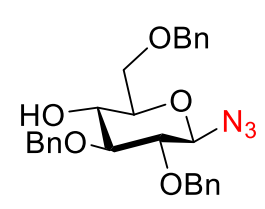

12
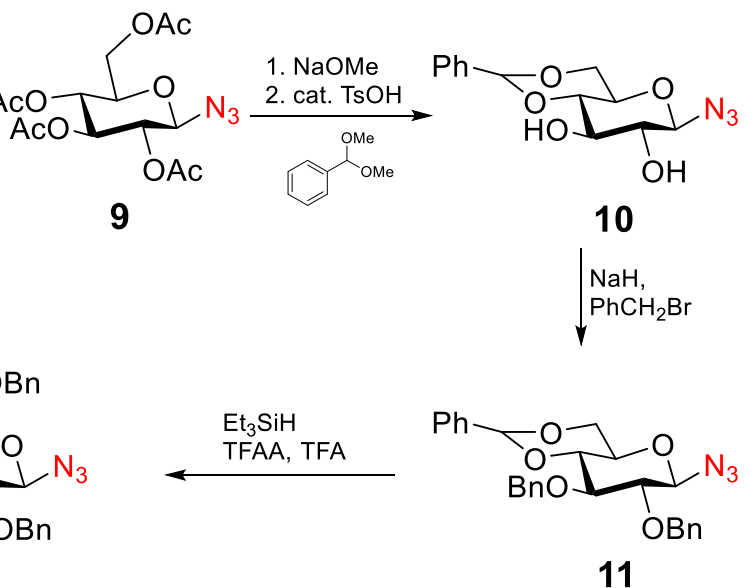

Scheme S1: Schematic representation for the synthesis of the glucose-derived monomer 1.

Glucose was firstly peracetylated and bromine was introduced at C1 in $\alpha$-configuration (8), which was replaced by an azide group in a following $S_{N} 2$-type nucleophilic substitution. ${ }^{1}$ Then, glucose derivative 9 with an azide group at C1 in $\beta$-configuration was deacetylated, leading to glucopyranosyl azide. ${ }^{2}$ In the following step, $\mathrm{C} 4$ and $\mathrm{C} 6$ of the glucopyranosyl azide were protected with benzaldehyde dimethyl acetal (10). ${ }^{3}$ The remaining hydroxyl groups at C2 and C3 were protected as benzyl ethers (11). ${ }^{2}$ The selective reductive cleavage of the acetal gave a free hydroxyl group at C4 (12), which has not been described before for such glucose derivative. Finally, the glucose-derived monomer $\mathbf{1}$ bearing an azide group at C1 in $\beta$-configuration and a propargyl group at C4 was obtained after the introduction of propargyl group that has been performed similar to the etherification of 10 to 11 . 


\section{2,3,4,6-Tetra-0-acetyl- $\alpha$-D-glucopyranosyl bromide (8)}

Glucose monohydrate $(11 \mathrm{~g}, 55.5 \mathrm{mmol})$ and $220 \mathrm{mg}$ iodine were added to $100 \mathrm{ml}$ acetic anhydride. The reaction mixture was stirred at $\mathrm{rt}$ for $4 \mathrm{~h}$. A red solution was obtained. Then, the reaction was quenched with $100 \mathrm{ml}$ of water, decolorized with a small amount of sodium thiosulfate and further stirred for $30 \mathrm{~min}$. The product was extracted with chloroform and the organic phase was washed until neutral with a solution of sodium bicarbonate. The organic solution was dried over sodium sulfate, filtrated and dried in vacuo to give the acetylated glucose. It was dissolved in $160 \mathrm{ml} \mathrm{DCM}$ and $65 \mathrm{ml}$ hydrobromic acid (33 wt.\% in acetic acid) were added. After stirring at rt for $3 \mathrm{~h}, 150 \mathrm{ml}$ water was added and the aqueous phase was extracted twice with $50 \mathrm{ml} \mathrm{DCM}$. The combined organic phases were washed with water and sodium bicarbonate solution until neutral. The organic solution was dried over sodium sulfate, filtrated, dried in vacuo and purified by using column chromatography in $n$ hexane/EA (2:1) to give $21.55 \mathrm{~g}$ ( $52.4 \mathrm{mmol} ; 94 \%$ yield) of the desired product.

$\mathrm{R}_{\mathrm{F}}=0.35$ in $n$-hexane/EA (2:1).

${ }^{1} \mathrm{H}$ NMR $\left(300 \mathrm{MHz}, \mathrm{CDCl}_{3}\right): \delta=6.62$ (d, J=4.0, 1H, H-1), 5.57 (t, J=9.7, $1 \mathrm{H}, \mathrm{H}-3$ ), 5.17 (dd, J=10.2, 9.4, $1 \mathrm{H}$, $\mathrm{H}-4$ ), 4.85 (dd, J=10.0, 4.1, 1H, H-2), 4.34 (m, 1H, H-5), 4.30 (m, 1H, H-6a), 4.14 (m, 1H, 6b), 2.11 (s, 3H, $\left.\mathrm{O}-\mathrm{CH}_{3}\right), 2.11\left(\mathrm{~s}, 4 \mathrm{H}, \mathrm{O}-\mathrm{CH}_{3}\right), 2.06\left(\mathrm{~s}, 3 \mathrm{H}, \mathrm{O}-\mathrm{CH}_{3}\right), 2.04\left(\mathrm{~s}, 3 \mathrm{H}, \mathrm{O}-\mathrm{CH}_{3}\right)$.

\section{2,3,4,6-Tetra-O-acetyl- $\beta$-D-glucopyranosyl azide (9)}

2,3,4,6-Tetra-O-acetyl- $\alpha$-D-glucopyranosyl bromide $(8,21.55 \mathrm{~g}, 52.4 \mathrm{mmol})$ was dissolved in $120 \mathrm{ml}$ $\mathrm{N}, \mathrm{N}$-dimethylsulfoxide and $5 \mathrm{~g}$ sodium azide $(77 \mathrm{mmol}, 1.5 \mathrm{eq}$ ) were added. After stirring for $30 \mathrm{~min}$ at $\mathrm{rt}, 100 \mathrm{ml}$ water and $100 \mathrm{ml}$ chloroform were added. The aqueous layer was extracted a second time with $100 \mathrm{ml}$ chloroform. The combined organic phase was washed with water (7x100 ml), dried over sodium sulfate, filtrated and dried in vacuo to give $17.47 \mathrm{~g}$ ( $46.8 \mathrm{mmol} ; 88 \%$ yield) of a pale yellow oil. It was used without further purification in the next reaction step.

${ }^{1} \mathrm{H}$ NMR $\left(300 \mathrm{MHz}, \mathrm{CDCl}_{3}\right): \delta=5.20$ (t, J=9.4, 1H, H-3), 5.08 (dd, J=10.0, 9.4, 1H, H-4), 4.93 (dd, J=9.4, 8.9, $1 \mathrm{H}, \mathrm{H}-2$ ), 4.63 (d, J=8.9, 1H, H-1), 4.25 (dd, J=12.5, 4.8, 1H, H-6a), 4.14 (dd, J=12.5, 2.4, 1H, H-6b), $3.78(\mathrm{ddd}, \mathrm{J}=9.9,4.7,2.4,1 \mathrm{H}, \mathrm{H}-5), 2.08\left(\mathrm{~s}, 3 \mathrm{H},-\mathrm{CH}_{3}\right), 2.05\left(\mathrm{~s}, 3 \mathrm{H},-\mathrm{CH}_{3}\right), 2.01\left(\mathrm{~s}, 3 \mathrm{H},-\mathrm{CH}_{3}\right), 1.98(\mathrm{~s}, 3 \mathrm{H},-$ $\left.\mathrm{CH}_{3}\right)$. 


\section{4,6-O-(phenyImethylene)- $\beta$-D-glucopyranosyl azide (10)}

2,3,4,6-Tetra-O-acetyl-B-D-glucopyranosyl azide $(9,17.47 \mathrm{~g}, 46.8 \mathrm{mmol})$ and $700 \mathrm{mg}$ sodium methoxide ( $13.5 \mathrm{mmol}, 0.3$ eq.) were dissolved in $200 \mathrm{ml}$ methanol and stirred for $90 \mathrm{~min}$ at $\mathrm{rt}$. The solution was filtrated over Dowex ion exchange resin and dried in vacuo. The intermediate was dissolved in $75 \mathrm{ml}$ of DMF, together with $10.51 \mathrm{ml}$ of benzaldehyde dimethyl acetal $(10.65 \mathrm{~g}$, $70.5 \mathrm{mmol}, 1.5$ eq.) and $420 \mathrm{mg} p$-toluenesulfonic acid. The reaction was conducted at $50{ }^{\circ} \mathrm{C}$ and $40 \mathrm{mbar}$ for $3 \mathrm{~h}$ on a rotary evaporator. After quenching the reaction with $2 \mathrm{ml}$ triethylamine, $100 \mathrm{ml}$ $E A$ and $100 \mathrm{ml}$ water were added. The aqueous phase was extracted twice with $50 \mathrm{ml} E A$, the combined organic phase was washed with brine $(4 \times 150 \mathrm{ml})$, dried over sodium sulfate, filtrated, dried in vacuo, dissolved in as little THF as possible and purified by using column chromatography in $n$-hexane/EA (3:2) to give $10.40 \mathrm{~g}$ ( $35.4 \mathrm{mmol}, 76 \%$ yield) of the desired product.

${ }^{1} \mathrm{H}$ NMR $\left(500 \mathrm{MHz}, \mathrm{CDCl}_{3}\right): \delta=7.49-7.35(\mathrm{~m}, 5 \mathrm{H}, \mathrm{Ar}-\mathrm{H}), 5.52(\mathrm{~s}, 1 \mathrm{H}, \mathrm{Ar}-\mathrm{CH}-)$, 4.66 (d, J=8.6, $\left.1 \mathrm{H}, \mathrm{H}-1\right)$, 4.37 (ddd, J=10.5, 3.5, 1.0, 1H, H-5), $3.83-3.67$ (m, 2H, H-3\&H-6a), $3.59-3.49$ (m, 2H, H-4\&H-6b), $3.43(\mathrm{t}, \mathrm{J}=8.7,1 \mathrm{H}, \mathrm{H}-2), 2.76(\mathrm{~s}, 1 \mathrm{H}, \mathrm{O}-3-H), 2.61(\mathrm{~s}, 1 \mathrm{H}, \mathrm{O}-2-H)$.

\section{2,3-Bis-O-benzyl-4,6-O-(phenylmethylene)- $\beta$-D-glucopyranosyl azide (11)}

4,6-O-(phenylmethylene)- $\beta$-D-glucopyranosyl azide $(\mathbf{1 0}, 10.40 \mathrm{~g}, 35.4 \mathrm{mmol}$ ) was dissolved in $120 \mathrm{ml}$ DMF and cooled to $0{ }^{\circ} \mathrm{C}$. Sodium hydride (4956 mg of a $60 \mathrm{wt} . \%$ dispersion in mineral oil, $124 \mathrm{mmol}$, 3.5 eq.) was slowly added and stirred at $0{ }^{\circ} \mathrm{C}$ for further $10 \mathrm{~min}$. Then, $12.6 \mathrm{ml}$ benzyl bromide ( 3 eq.) were slowly added and the solution was stirred at $0{ }^{\circ} \mathrm{C}$ for further $30 \mathrm{~min}$. The solution was allowed to warm up to rt. TLC control indicates that the reaction was finished after $3 \mathrm{~h}$. By carful and slow addition of $100 \mathrm{ml}$ water, the reaction was stopped. The product was extracted with EA $(2 \times 100 \mathrm{ml})$ and the combined organic phase was washed with water $(2 \times 200 \mathrm{ml})$ and brine $(6 \times 100 \mathrm{ml})$, dried over sodium sulfate, filtrated, dried in vacuo and purified by using column chromatography in $n$-hexane/EA (17:3) to give $14.75 \mathrm{~g}$ ( $31.1 \mathrm{mmol}, 88 \%$ yield) of the dibenzylether 11 as product.

$\mathrm{R}_{\mathrm{F}}=0.40$ in $n$-hexane/EA (17:3).

${ }^{1} \mathrm{H}$ NMR $\left(500 \mathrm{MHz}, \mathrm{CDCl}_{3}\right): \delta=7.50-7.26(\mathrm{~m}, 15 \mathrm{H}, \mathrm{Ar}-\mathrm{H}), 5.57(\mathrm{~s}, 1 \mathrm{H}, \mathrm{Ar}-\mathrm{CH}-)$ ) 4.93 (d, J=11.3, 1H, O-3$\mathrm{CH}_{2} \mathrm{a}$ ), $4.83\left(\mathrm{~s}, 2 \mathrm{H}, \mathrm{O}-2-\mathrm{CH}_{2}\right), 4.78$ (d, J=11.3, 1H, O-3- $\mathrm{CH}_{2} \mathrm{~b}$ ), 4.72 (d, J=8.5, 1H, H-1), 4.38 (dd, J=10.5, 5.0, $1 \mathrm{H}, \mathrm{H}-6 \mathrm{a}$ ), 3.78 (dd, J=9.3, 8.7, 1H, H-3), 3.78 (t, J=10.3, 1H, H-6b), 3.69 (t, J=9.3, 1H, H-4), 3.50 (ddd, J=10.1, 9.4, 5.0, 1H, H-5), 3.38 (t, J=8.6, $1 \mathrm{H}, \mathrm{H}-2$ ). 


\section{2,3,6-Tris-O-benzyl- $\beta$-D-glucopyranosyl azide (12)}

2,3-Bis-O-benzyl-4,6-O-(phenylmethylene)- $\beta$-D-glucopyranosyl azide $(\mathbf{1 1}, 14.75 \mathrm{~g}, 31.1 \mathrm{mmol})$ was dissolved in $350 \mathrm{ml} \mathrm{DCM}$ and cooled to $0{ }^{\circ} \mathrm{C}$. Subsequently, triethylsilane (24.84 ml, $155 \mathrm{mmol}, 5$ eq.), trifluoroacetic anhydride (12.98 ml, $93.3 \mathrm{mmol}, 3$ eq.) and trifluoroacetic acid (11.98 ml, $155.5 \mathrm{mmol}$, 5 eq.) were added carefully. The solution was slowly allowed to warm to rt. TLC control indicated that the reaction had been finished after $7 \mathrm{~h}$. After washing the reaction solution with sodium bicarbonate solution $(6 \times 100 \mathrm{ml})$, it was dried over sodium sulfate, filtrated, dried in vacuo and purified by using column chromatography in $n$-hexane/EA (4:1) to give $11.16 \mathrm{~g}$ ( $23.5 \mathrm{mmol}, 75 \%$ yield) of 12.

$\mathrm{R}_{\mathrm{F}}=0.2$ in $n$-hexane/EA (4:1).

${ }^{1} \mathrm{H}$ NMR $\left(500 \mathrm{MHz}, \mathrm{CDCl}_{3}\right): \delta=7.39-7.25(\mathrm{~m}, 15 \mathrm{H}, \mathrm{Ar}-\mathrm{H}), 4.91\left(\mathrm{~d}, \mathrm{~J}=11.4,1 \mathrm{H}, \mathrm{CH}_{2}-\mathrm{a}\right), 4.88(\mathrm{~d}, \mathrm{~J}=10.8$, $\left.1 \mathrm{H}, \mathrm{C}^{\prime} \mathrm{H}_{2}-\mathrm{a}\right), 4.76$ (d, J=11.6, 1H, $\left.\mathrm{CH}_{2}-\mathrm{b}\right), 4.76$ (d, J=10.6, 1H, C'H $\left.\mathrm{H}_{2}-\mathrm{b}\right), 4.64$ (s, 1H, H-1), 4.61 (d, J=12.2, $1 \mathrm{H}, \mathrm{C}^{\prime \prime} \mathrm{H}_{2}-\mathrm{a}$ ), 4.56 (d, J=12.1, 1H, C"H $\mathrm{H}_{2}-\mathrm{b}$ ), 3.76 (dd, J=10.6, 3.9, 1H, H-6a), 3.72 (dd, J=10.6, 4.9, 1H, H6b), 3.63 (dd, J=9.7, 8.9, 1H, H-4), 3.51 (ddd, J=9.7, 4.9, 3.9, 1H, H-5), 3.47 (t, J=9.0, 1H, H-3), 3.34 (dd, J=9.1, 8.6, $1 \mathrm{H}, \mathrm{H}-2), 2.54(\mathrm{~s}, 1 \mathrm{H},-\mathrm{OH})$.

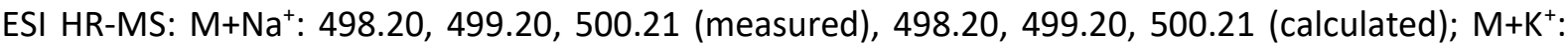
514.17, 515.18, 516.17 (measured), 514.17, 515.18, 516.17 (calculated).

\section{2,3,6-Tris-O-benzyl-4-propargyl- $\beta$-D-glucopyranosyl azide (1)}

2,3,6-Tris-O-benzyl- $\beta$-D-glucopyranosyl azide $(14,1 \mathrm{~g}, 2.06 \mathrm{mmol})$ was dissolved in $8 \mathrm{ml} \mathrm{DMF}$, cooled to $0{ }^{\circ} \mathrm{C}$ and sodium hydride ( $165 \mathrm{mg}, 60 \mathrm{wt} . \%$ dispersion in mineral oil, 2 eq.) was slowly added. After stirring for $10 \mathrm{~min}$ at $0{ }^{\circ} \mathrm{C}, 293 \mu \mathrm{l}$ propargyl bromide (80\% in toluene, 1.5 eq.) was slowly added and the reaction was conducted for $30 \mathrm{~min}$ at $0{ }^{\circ} \mathrm{C}$. Then, it was allowed to slowly warm up to rt. After 90 min the reaction was finished. Remaining sodium hydride was carefully hydrolyzed via the addition of water $(10 \mathrm{ml})$. The product was extracted twice with $30 \mathrm{ml}$ chloroform, washed with brine $(6 \times 50 \mathrm{ml})$, dried over sodium sulfate, filtrated, concentrated in vacuo and purified by using column chromatography in $n$-hexane/EA (6:1) to give $930 \mathrm{mg}(1.81 \mathrm{mmol}, 88 \%$ yield) of the desired product. The product was stored as a solution in THF.

$\mathrm{R}_{\mathrm{F}}=0.3$ in $n$-hexane/EA (6:1).

${ }^{1} \mathrm{H} \mathrm{NMR}\left(500 \mathrm{MHz}, \mathrm{CDCl}_{3}\right): \delta=7.36-7.25(\mathrm{~m}, 15 \mathrm{H}, \mathrm{Ar}-\mathrm{H}), 4.87\left(\mathrm{~d}, \mathrm{~J}=10.9,1 \mathrm{H}, \mathrm{Ar}-\mathrm{CH}_{2}-\mathrm{a}\right), 4.86$ (d, J=10.7, $1 \mathrm{H}, \operatorname{Ar}-\mathrm{C}^{\prime} \mathrm{H}_{2}-\mathrm{a}$ ), 4.81 (d, J=10.8, 1H, Ar- $\mathrm{CH}_{2}-\mathrm{b}$ ), 4.73 (d, J=10.7, 1H, Ar-C' $\mathrm{H}_{2}-\mathrm{b}$ ), 4.63 (d, J=12.1, 1H, Ar$\mathrm{C}^{\prime \prime} \mathrm{H}_{2}-\mathrm{a}$ ), 4.60 (d, J=8.6, 1H, H-1), 4.57 (d, J=12.1, 1H, Ar-C"H $\mathrm{H}_{2}-\mathrm{b}$ ), 4.35 (dd, J=15.3, 2.4, 1H, HCCCH 4.25 (dd, J=15.3, 2.4, 1H, HCCCH $-a$ ), 3.82 (dd, J=11.1, 1.8, 1H, H-6b), 3.72 (dd, J=11.1, 4.4, 1H, H-6a), 
$3.60(t, J=8.8,1 H, H-3), 3.53(d d, J=9.6,8.6,1 H, H-4), 3.49(d d d, J=9.6,4.3,1.6,1 H, H-5), 3.32(t, J=8.8$, $1 \mathrm{H}, \mathrm{H}-2), 2.40(\mathrm{t}, \mathrm{J}=2.4,1 \mathrm{H}, \mathrm{CH})$.

${ }^{13} \mathrm{C}$ NMR (126 MHz, $\left.\mathrm{CDCl}_{3}\right): \delta=138.37$ - 125.91 (15C, Ar), 90.09 (C1), 84.59 (C3), 81.59 (C2), 79.63 $\left(\mathrm{HCCCH}_{2}\right), 76.86(\mathrm{C} 4), 76.72(\mathrm{C} 5), 75.75\left(\mathrm{Ar}-\mathrm{CH}_{2}\right), 75.15\left(\mathrm{Ar}-\mathrm{C}^{\prime} \mathrm{H}_{2}\right), 74.64(\mathrm{CCH}), 73.54\left(\mathrm{Ar}-\mathrm{C}^{\prime \prime} \mathrm{H}_{2}\right), 68.60$ (C6), $59.95\left(\mathrm{HCCCH}_{2}\right)$.

ESI HR-MS: $\mathrm{M}+\mathrm{NH}_{4}^{+}$: 531.26, 532.26, 533.26 (measured), 531.26, 532.26, 533.27 (calculated); $\mathrm{M}+\mathrm{Na}{ }^{+}$: 536.22, 537.22, 538.22 (measured), 536.22, 537.22, 538.22 (calculated).

\section{Sythesis of azide-modified silica nanoparticles}

\section{3-Azido-1-propanol (6)}

3-Bromo-1-propanol (5.86 ml, $64.8 \mathrm{mmol})$ and sodium azide ( $8.39 \mathrm{~g}, 129 \mathrm{mmol}$; 2 eq.) were dissolved in $90 \mathrm{ml}$ water and stirred at $80^{\circ} \mathrm{C}$ for $72 \mathrm{~h}$. Then, the solution was cooled down to rt and extracted with DCM (3x100 ml), dried over sodium sulfate, filtrated and dried in vacuo to give $5.56 \mathrm{~g}$ (54.9 mmol, $85 \%$ yield) of a colorless liquid.

${ }^{1} \mathrm{H} \mathrm{NMR}\left(300 \mathrm{MHz} \mathrm{CDCl}_{3}\right): \delta=3.75(\mathrm{t}, J=6.0,2 \mathrm{H}), 3.45(\mathrm{t}, J=6.6,2 \mathrm{H}), 1.83(\mathrm{p}, J=6.3,2 \mathrm{H}), 1.70(\mathrm{~s}, 1 \mathrm{H})$.

\section{3-Azidopropyl undec-10-enoate (7)}

3-Azido-1-propanol (5.56 g; 54,9 mmol) was dissolved in $190 \mathrm{ml} \mathrm{DCM}$, cooled to $0{ }^{\circ} \mathrm{C}$ and $26.64 \mathrm{ml}$ triethylamine (3.5 eq.) was added. Then, $11.84 \mathrm{ml} 10$-undecenoyl chloride (11.13 g, 1 eq.) were slowly dropped into the solution. The reaction solution was allowed to warm to rt and stirred overnight. It was washed with brine $(5 \times 100 \mathrm{ml})$, dried over sodium sulfate, filtrated, dried in vacuo and purified by using column chromatography in $n$-hexane/EA (6:1) to give $10.9 \mathrm{~g}$ ( $40.8 \mathrm{mmol}, 74 \%$ yield) of the ester.

${ }^{1} \mathrm{H} \mathrm{NMR}\left(300 \mathrm{MHz}, \mathrm{CDCl}_{3}\right): \delta=5.81(\mathrm{ddt}, 1 \mathrm{H},=\mathrm{CH}-), 5.06-4.87\left(\mathrm{~m}, 2 \mathrm{H},=\mathrm{CH}_{2}\right), 4.16\left(\mathrm{t}, 2 \mathrm{H}, \mathrm{O}-\mathrm{CH}_{2}\right), 3.39$ (t, $2 \mathrm{H},-\mathrm{CH}_{2} \mathrm{~N}_{3}$ ), 2.31 (t, 2H, CH $\left.2-\mathrm{COO}\right), 2.04$ (tdd, $2 \mathrm{H},=\mathrm{CH}_{2}-\mathrm{CH}_{2}$ ), 1.91 (p, 2H, $\left.\mathrm{CH}_{2}-\mathrm{CH}_{2} \mathrm{~N}_{3}\right), 1.62$ (t, J=7.3, $\left.2 \mathrm{H}, \mathrm{CH}_{2}-\mathrm{CH}_{2}-\mathrm{COO}\right), 1.43-1.22\left(\mathrm{~m}, 10 \mathrm{H},-\left(\mathrm{CH}_{2}\right)_{5}-\right)$.

\section{3-Azidopropyl 11-(chlorodimethylsilyl)undecanoate (2)}

3-Azidopropyl undec-10-enoate ( $4 \mathrm{~g}, 14.9 \mathrm{mmol}$ ) was dissolved in $40 \mathrm{ml}$ chlorodimethylsilane, $60 \mathrm{mg}$ hexachloroplatinic acid in $0.5 \mathrm{ml}$ 2-propanol were added and the solution was heated to reflux for $3 \mathrm{~h}$. Unreacted chlorodimethylsilane was removed by destillation. DCM $(20 \mathrm{ml})$ was added to the remaining 
yellowish solution. After that, the solution was filtrated over sodium sulfat to remove the catalyst and dried in vacuo to give $4.92 \mathrm{~g}$ of the chlorosilane ( $13.6 \mathrm{mmol}, 91 \%$ yield).

${ }^{1} \mathrm{H}$ NMR $\left(300 \mathrm{MHz}, \mathrm{CDCl}_{3}\right) \delta=4.16\left(\mathrm{t}, 2 \mathrm{H}, \mathrm{O}-\mathrm{CH}_{2}\right), 3.40\left(\mathrm{t}, 2 \mathrm{H}, \mathrm{CH}_{2} \mathrm{~N}_{3}\right), 2.31\left(\mathrm{t}, 2 \mathrm{H}, \mathrm{CH}_{2}-\mathrm{COO}\right), 1.92(\mathrm{p}$, $\left.2 \mathrm{H}, \mathrm{CH}_{2}-\mathrm{CH}_{2} \mathrm{~N}_{3}\right), 1.62\left(\mathrm{p}, 2 \mathrm{H}, \mathrm{CH}_{2}-\mathrm{CH}_{2} \mathrm{COO}\right), 1.46-1.23\left(\mathrm{~m}, 14 \mathrm{H},-\left(\mathrm{CH}_{2}\right)_{7}-\right), 0.82\left(\mathrm{~m}, 2 \mathrm{H}, \mathrm{Si}-\mathrm{CH}_{2}\right), 0.40$ (s, $\left.6 \mathrm{H}, \mathrm{Si}-\left(\mathrm{CH}_{3}\right)_{2}\right)$.

\section{Surface-modified silica nanoparticles with azide groups (SiNPs-N $\mathrm{N}_{3}$ )}

SiNPs with a specific BET-surface of $49.25 \mathrm{~m}^{2} / \mathrm{g}$ were dried at $180^{\circ} \mathrm{C}$ and $0.01 \mathrm{mbar}$ for $8 \mathrm{~h}$. Then, they were dispersed in a mixture of $150 \mathrm{ml}$ toluene and $15 \mathrm{ml}$ pyridine, before $4.92 \mathrm{~g}$ 3-azidopropyl 11-(chlorodimethylsilyl)undecanoate were added. After continuous stirring at rt for $16 \mathrm{~h}$, modified SiNPs were washed with toluene, ethanol, ethanol/water $(1: 1, \mathrm{pH}=3)$, ethanol/water $(1: 1)$, ethanol and diethyl ether, and dried in vacuo.

Elemental analysis in wt-\%: C: 3.50; H: 1.20; N: 0.46.

Density of azide groups: $109.5 \mu \mathrm{mol} / \mathrm{g}$ or $2.22 \mu \mathrm{mol} / \mathrm{m}^{2}$.

\section{Click reaction}

\section{General procedure}

The azide-bearing SiNPs- $\mathrm{N}_{3}$ ( $200 \mathrm{mg} ; 21.9 \mu \mathrm{mol} \mathrm{N}$-groups) were dispersed in $16 \mathrm{ml} \mathrm{THF}$ and $5.44 \mathrm{ml}$ DIPEA (32 mmol). After degassing for $20 \mathrm{~min}$, the catalyst copper(I)-iodide was added $(15.2 \mathrm{mg}$; $0.08 \mathrm{mmol}$ ). Then, the freshly prepared 2,3,6-tris-O-benzyl-4-propargyl- $\beta$-D-glucopyranosyl azide (1, $30 \mathrm{wt}-\%$ in THF) was added as described below. After the addition was completed, the dispersion was stirred at $\mathrm{rt}$ for $24 \mathrm{~h}$. Then, modified SiNPs were separated by centrifugation, washed 5 times with THF, one time with acetonitrile and dried in vacuo.

The supernatant solution and the washing solutions were combined and the solvent was distilled off. The organic residue was dissolved in chloroform, extracted with brine, dried over sodium sulfate, filtered and dried in vacuo to give the cyclic compounds.

\section{CuAAc with 20 eq. monomer}

2,3,6-Tris-O-benzyl-4-propargyl- $\beta$-D-glucopyranosyl azide 1 (225 mg, $438 \mu \mathrm{mol}$ ) dissolved in $600 \mu \mathrm{l} \mathrm{THF}$ were added in one step. 
Elemental analysis of SiNPs-P 20 in wt.\%: C: 13.90; $\mathrm{H}: 1.86 ; \mathrm{N}: 1.73$.

DP calculated based on the results from elemental analysis: 3.46 .

${ }^{1} \mathrm{H}$ NMR $\left(300 \mathrm{MHz}, \mathrm{CDCl}_{3}\right): \delta=7.30(\mathrm{~m}, 1 \mathrm{H}$, triazole- $H)$, 7.36-7.18 $(\mathrm{m}, 10 \mathrm{H}, \mathrm{Ar}-\mathrm{H}), 7.14(\mathrm{~m}, 3 \mathrm{H}, \mathrm{Ar}-\mathrm{H})$, $6.90(\mathrm{~m}, 2 \mathrm{H}, \mathrm{Ar}-\mathrm{H}), 5.46$ (d, J=9.1, 1H, H-1), 4.98 (d, J=11.9, $\left.1 \mathrm{H}, \mathrm{Ar}^{-} \mathrm{C}^{1} \mathrm{H}_{2}-\mathrm{a}\right), 4.91$ (s, 2H, Ar- $\left.\mathrm{C}^{2} \mathrm{H}_{2}\right), 4.73$

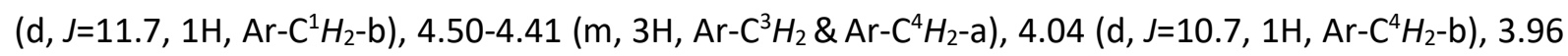
(m, $1 \mathrm{H}, \mathrm{H}-2), 3.81-3.61(\mathrm{~m}, 5 \mathrm{H}, \mathrm{H}-4$ \& $\mathrm{H}-3$ \& $\mathrm{H}-6$ \& $\mathrm{H}-5)$.

\section{CuAAc with 80 eq. monomer}

2,3,6-Tris-O-benzyl-4-propargyl- $\beta$-D-glucopyranosyl azide 1 (900 mg, $1752 \mu \mathrm{mol}$ ) dissolved in $1800 \mu \mathrm{l}$ THF were added in one step.

Elemental analysis of SiNPs- $\mathrm{P}_{80}$ in wt.\%: C: $21.30 ; \mathrm{H}: 2.45 ; \mathrm{N}: 2.57$.

DP calculated based on the results from elemental analysis: 6.62 .

${ }^{1} \mathrm{H}$ NMR $\left(300 \mathrm{MHz}, \mathrm{CDCl}_{3}\right): \delta=7.30(\mathrm{~m}, 1 \mathrm{H}$, triazole- $H)$, 7.36-7.18 $(\mathrm{m}, 10 \mathrm{H}, \mathrm{Ar}-\mathrm{H}), 7.14(\mathrm{~m}, 3 \mathrm{H}, \mathrm{Ar}-\mathrm{H})$, $6.90(\mathrm{~m}, 2 \mathrm{H}, \mathrm{Ar}-\mathrm{H}), 5.46$ (d, J=9.1, 1H, H-1), 4.98 (d, J=12.6, 1H, Ar- $\left.\mathrm{C}^{1} \mathrm{H}_{2}-\mathrm{a}\right), 4.89$ (s, 2H, Ar- $\left.\mathrm{C}^{2} \mathrm{H}_{2}\right), 4.74$ (d, J=11.7, $\left.1 \mathrm{H}, \operatorname{Ar}-\mathrm{C}^{1} \mathrm{H}_{2}-\mathrm{b}\right), 4.48\left(\mathrm{~m}, 2 \mathrm{H}, \mathrm{Ar}-\mathrm{C}^{3} \mathrm{H}_{2}\right.$ ), 4.43 (s, $\left.1 \mathrm{H}, \operatorname{Ar}-\mathrm{C}^{4} \mathrm{H}_{2}-\mathrm{a}\right), 4.04$ (d, J=10.7, $1 \mathrm{H}, \mathrm{Ar}-\mathrm{C}^{4} \mathrm{H}_{2}-\mathrm{b}$ ), $3.96(m, 1 H, H-2), 3.81-3.61(m, 5 H, H-4$ \& H-3 \& H-6 \& H-5).

\section{CuAAc with stepwise addition of 77 eq. monomer}

2,3,6-Tris-O-benzyl-4-propargyl- $\beta$-D-glucopyranosyl azide 1 (870 mg, $1686 \mu \mathrm{mol}$ ) dissolved in $1800 \mu \mathrm{l}$ THF was added once per hour with one seventh of the solution.

Elemental analysis of SiNPs-P $\mathrm{P}_{77}$ in wt.\%: $\mathrm{C}: 24.80 ; \mathrm{H}: 2.70 ; \mathrm{N}: 2.96$.

DP calculated based on the results from elemental analysis: 8.44 .

${ }^{1} \mathrm{H}$ NMR $\left(500 \mathrm{MHz}, \mathrm{CDCl}_{3}\right): \delta=7.30(\mathrm{~m}, 1 \mathrm{H}$, triazole- $H)$, 7.36-7.18 $(\mathrm{m}, 10 \mathrm{H}, \mathrm{Ar}-H), 7.14(\mathrm{~m}, 3 \mathrm{H}, \mathrm{Ar}-H)$, $6.90(\mathrm{~m}, 2 \mathrm{H}, \mathrm{Ar}-\mathrm{H}), 5.45$ (d, J=8.9, $1 \mathrm{H}, \mathrm{H}-1), 4.97$ (d, J=11.8, 1H, Ar-C $\left.\mathrm{C}_{2}-\mathrm{a}\right), 4.89\left(\mathrm{~m}, 2 \mathrm{H}, \operatorname{Ar}-\mathrm{C}^{2} \mathrm{H}_{2}\right), 4.72$ (d, J=11.8, 1H, Ar-C $\left.{ }^{1} H_{2}-b\right), 4.49$ (m, 2H, Ar-C $C^{3} H_{2}$ ), 4.44 (d, J=10.7, 1H, Ar-C $\left.\mathrm{C}^{4} \mathrm{H}_{2}-\mathrm{a}\right), 4.03$ (d, J=10.6, 1H, Ar$\left.\mathrm{C}^{4} \mathrm{H}_{2}-\mathrm{b}\right), 3.96(\mathrm{t}, J=8.9,1 \mathrm{H}, \mathrm{H}-2), 3.81$ (d, J=9.3, 1H, H-4), 3.76 (m, 1H, H-3), 3.74 (m, 1H, H-6a), 3.68 (m, $1 \mathrm{H}, \mathrm{H}-6 b), 3.61(\mathrm{~m}, 1 \mathrm{H}, \mathrm{H}-5)$. 
${ }^{13} \mathrm{C}$ NMR (126 MHz, CDCl $)$ ): $\delta$ = 144.72 (triazole-C4), 138.03-136.93 (3C, ipso-C), 128.50-127,73 (15C, ortho-C, meta-C, para-C), 122.03 (triazole-C5), 87.42 (C1), 85.15 (C3), 80.54 (C2), 77.84 (C5), 77.36 (C4), $75.69\left(\operatorname{Ar}-C^{2} \mathrm{H}_{2}\right), 74.79\left(\operatorname{Ar}-C^{4} \mathrm{H}_{2}\right), 73.46\left(\operatorname{Ar}-C^{3} \mathrm{H}_{2}\right), 68.32(\mathrm{C} 6), 66.07\left(\operatorname{Ar}-\mathrm{C}^{1} \mathrm{H}_{2}\right)$.

\section{CuAAc with continuous addition of 80 eq. monomer}

2,3,6-tris-O-benzyl-4-propargyl- $\beta$-D-glucopyranosyl azide 1 (900 mg, $1752 \mu \mathrm{mol}$ ) dissolved in $1800 \mu \mathrm{l}$ THF was added continuously over $24 \mathrm{~h}$ by using a syringe pump (NE-1010 by New Era Pump Systems Inc).

Elemental analysis of SiNPs-P $80 \mathrm{c}$ in wt.\%: C: $31.75 ; \mathrm{H}: 3.24 ; \mathrm{N}: 3.73$.

DP calculated based on the results from elemental analysis: 13.01.

${ }^{1} \mathrm{H}$ NMR $\left(300 \mathrm{MHz}, \mathrm{CDCl}_{3}\right): \delta=7.30(\mathrm{~m}, 1 \mathrm{H}$, triazole- $H)$, 7.36-7.18 $(\mathrm{m}, 10 \mathrm{H}, \mathrm{Ar}-\mathrm{H}), 7.14(\mathrm{~m}, 3 \mathrm{H}, \mathrm{Ar}-\mathrm{H})$, $6.90(\mathrm{~m}, 2 \mathrm{H}, \mathrm{Ar}-\mathrm{H}), 5.46(\mathrm{~d}, \mathrm{~J}=9.0,1 \mathrm{H}, \mathrm{H}-1), 4.98\left(\mathrm{~d}, \mathrm{~J}=11.9,1 \mathrm{H}, \mathrm{Ar}^{-} \mathrm{C}^{1} \mathrm{H}_{2}-\mathrm{a}\right), 4.91$ (s, 2H, Ar-C $\left.\mathrm{C}^{2} \mathrm{H}_{2}\right), 4.73$

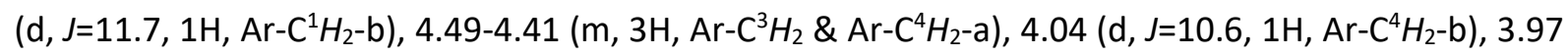
(m, $1 \mathrm{H}, \mathrm{H}-2), 3.81-3.61(\mathrm{~m}, 5 \mathrm{H}, \mathrm{H}-4$ \& $\mathrm{H}-3$ \& $\mathrm{H}-6$ \& H-5). 


\section{Calculation of DP from the elemental composition}

1. Organic content of the azide-bearing silica nanoparticles (SiNPs-N ${ }_{3}$ ) was calculated based on the mass fractions of carbon, hydrogen, nitrogen and organic bound oxygen (calculated from molecular formula, based on nitrogen content).

NP-N $\mathrm{N}_{3}: \quad 5.51 \%$ organic content

$94.49 \%$ silica

2. Organic contents of grafting products were calculated, similar to step 1.

$\begin{array}{ll}\text { SiNPs-P }_{20} & 20.26 \% \text { organic content } \\ \text { SiNPs-P }_{80} & 30.69 \% \text { organic content } \\ \text { SiNPs-P }_{77} & 35.57 \% \text { organic content } \\ \text { SiNPs-P }_{80 C} & 45.30 \% \text { organic content }\end{array}$

3. DP-calculation

\begin{tabular}{|c|c|c|c|c|}
\hline & $\begin{array}{l}\text { Organic } \\
\text { content }\end{array}$ & $\begin{array}{l}\text { Nitrogen } \\
\text { content }\end{array}$ & Nitrogen content from linker & Degree of polymerization \\
\hline $\mathrm{SiNPs}_{\mathrm{N}} \mathrm{N}_{3}$ & $5.51 \%$ & $0.46 \%$ & $0.46 \%$ & \\
\hline SiNPs- $P_{20}$ & $20.26 \%$ & $1.73 \%$ & $0.46 \% \cdot \frac{1-20.26 \%}{1-5.51 \%}=0.39 \%$ & $\frac{1.73 \%-0.39 \%}{0.39 \%}=3.46$ \\
\hline SiNPs-P 80 & $30.69 \%$ & $2.57 \%$ & $0.46 \% \cdot \frac{1-30.69 \%}{1-5.51 \%}=0.34 \%$ & $\frac{2.57 \%-0.34 \%}{0.34 \%}=6.62$ \\
\hline SiNPs-P 77 & $35.57 \%$ & $2.96 \%$ & $0.46 \% \cdot \frac{1-35.57 \%}{1-5.51 \%}=0.31 \%$ & $\frac{2.96 \%-0.31 \%}{0.31 \%}=8.44$ \\
\hline SiNPs- $P_{80 C}$ & $45.30 \%$ & $3.73 \%$ & $0.46 \% \cdot \frac{1-45.30 \%}{1-5.51 \%}=0.27 \%$ & $\frac{3.73 \%-0.27 \%}{0.27 \%}=13.01$ \\
\hline
\end{tabular}




\section{Compound 1: Spectroscopic data}

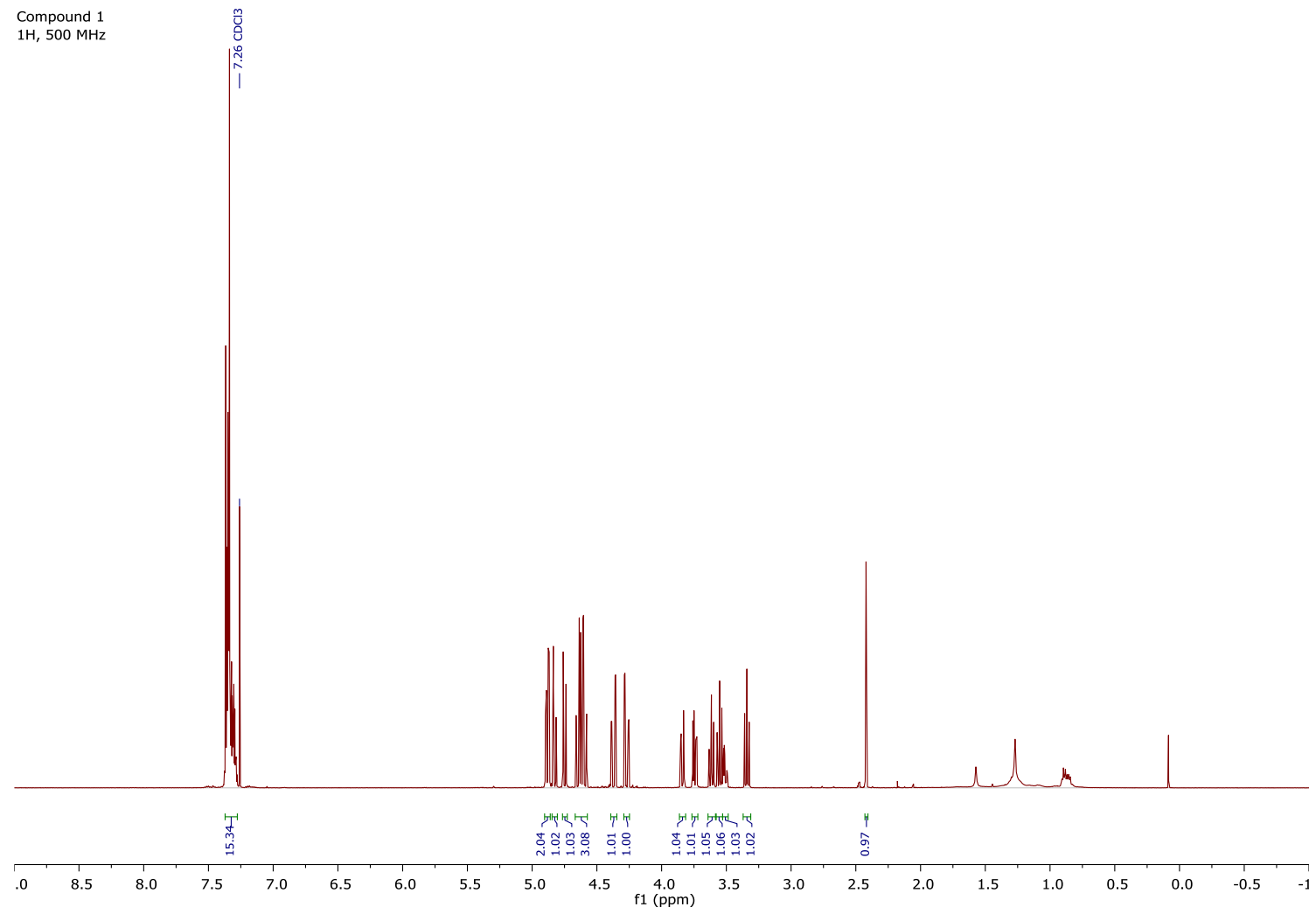

Compound 1
$13 \mathrm{C}, 125 \mathrm{MH}$

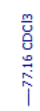

$-32000$

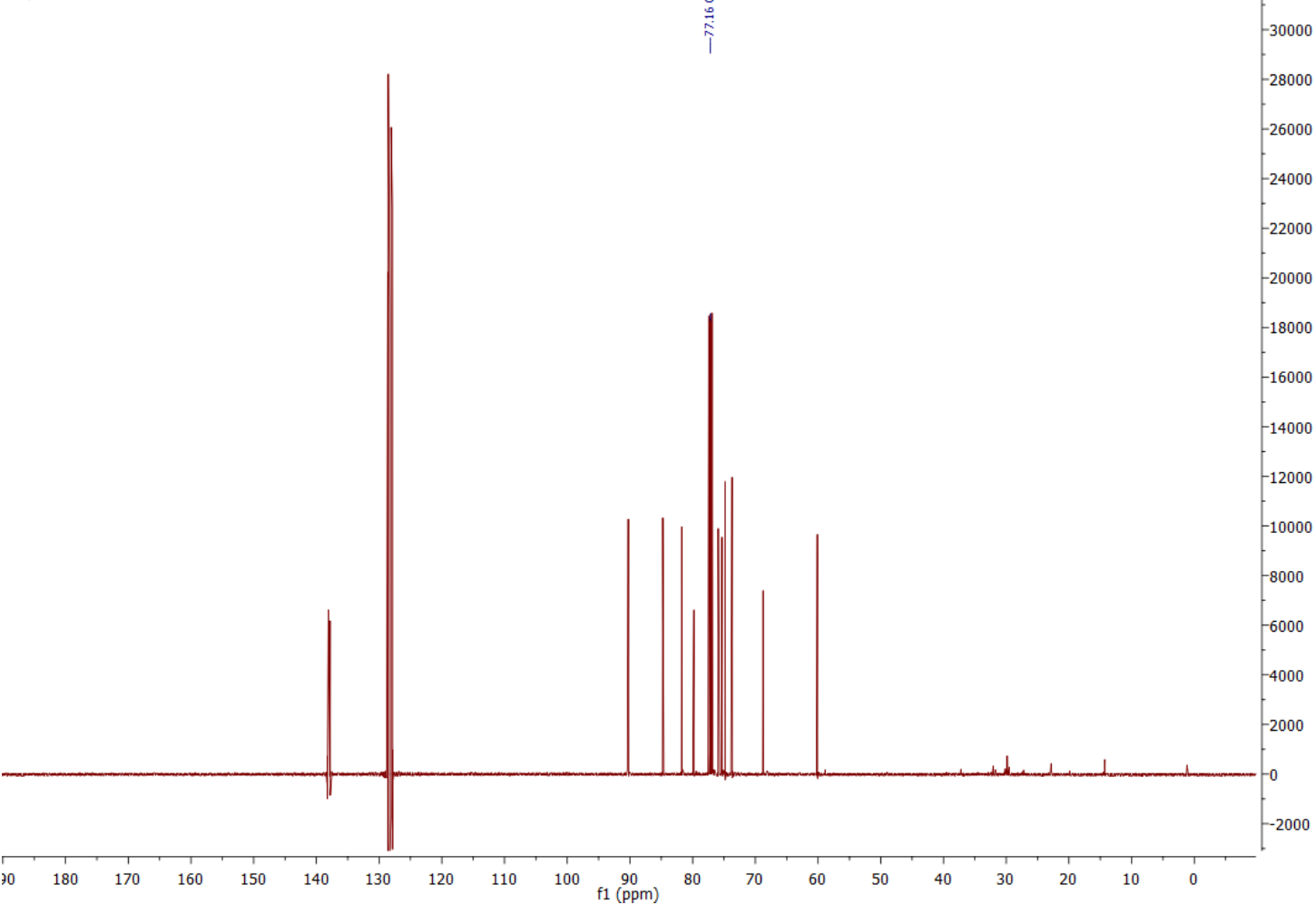



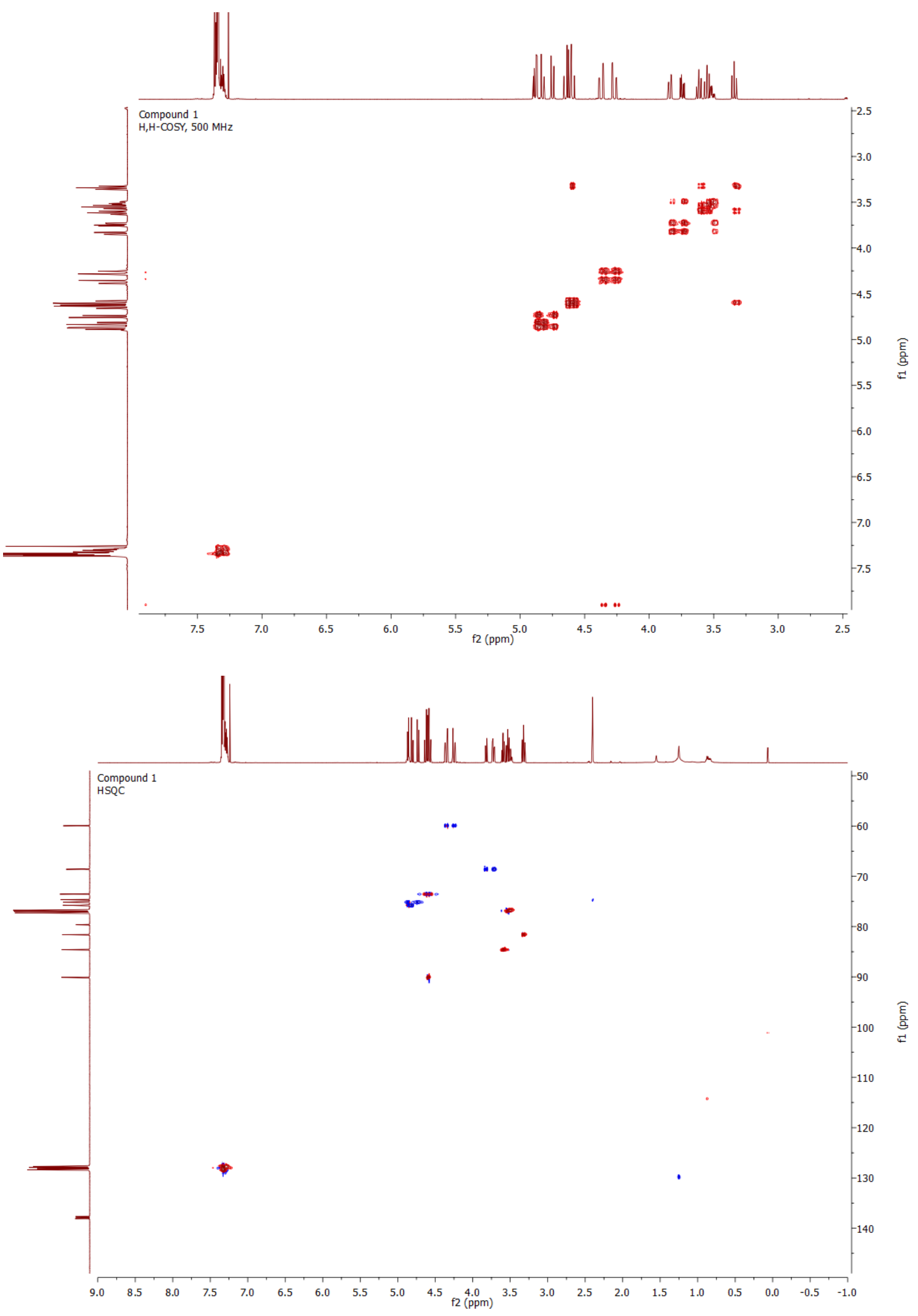
Compound 2: Spectroscopic data

Compound 2 $1 \mathrm{H}, 500 \mathrm{MHz}$

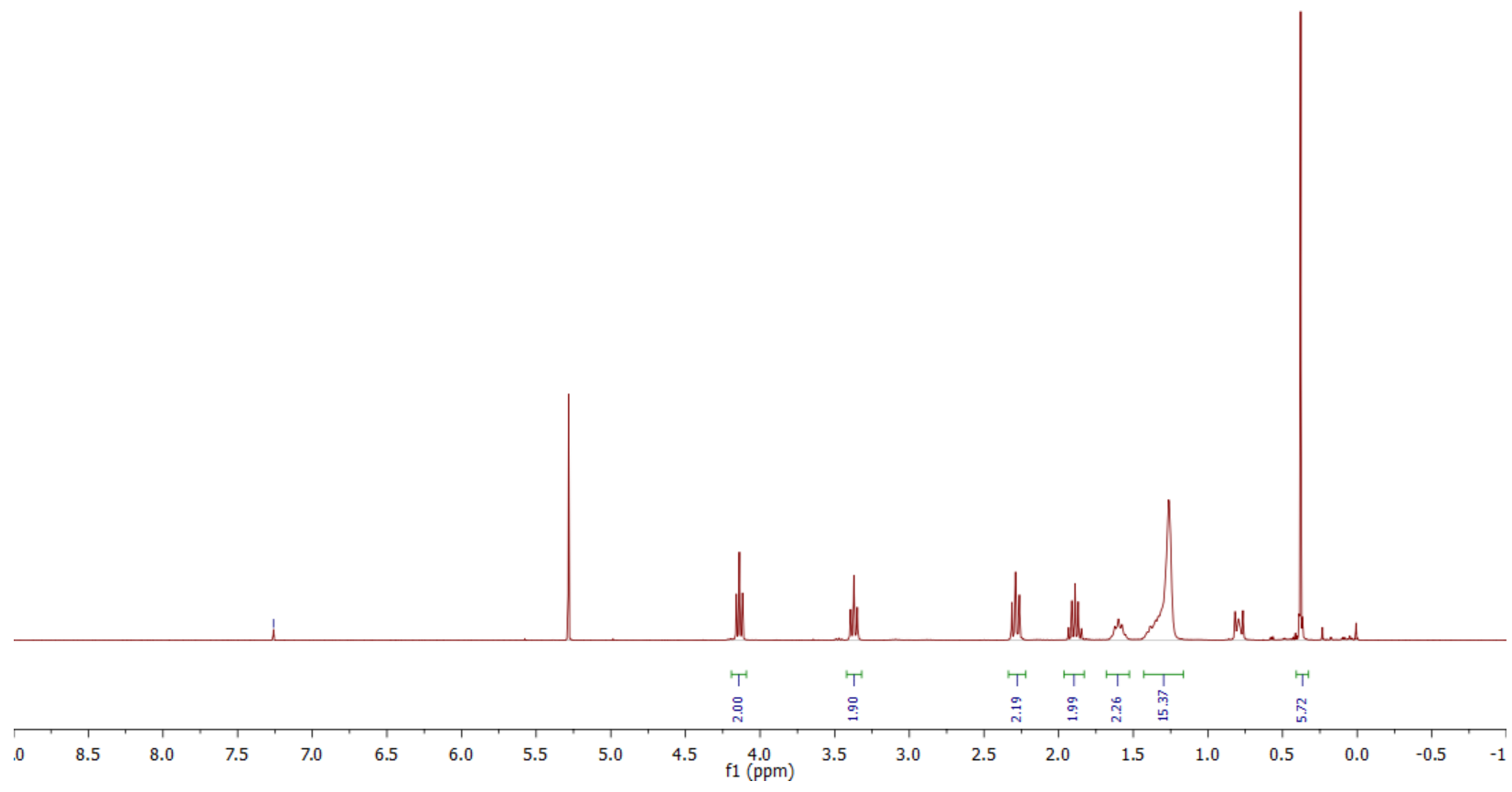


Compound 7: Spectroscopic data
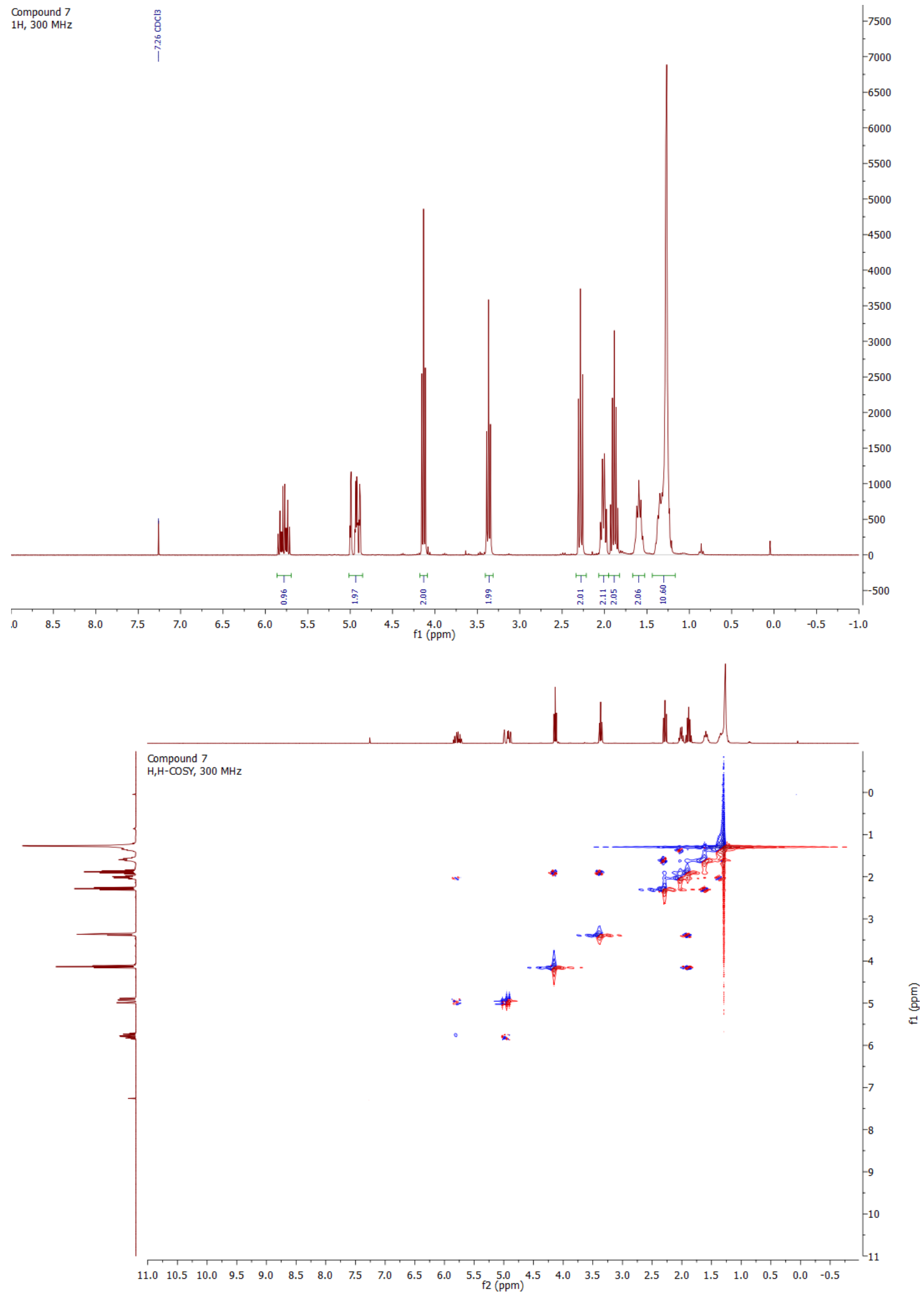
Compound 12: Spectroscopic data
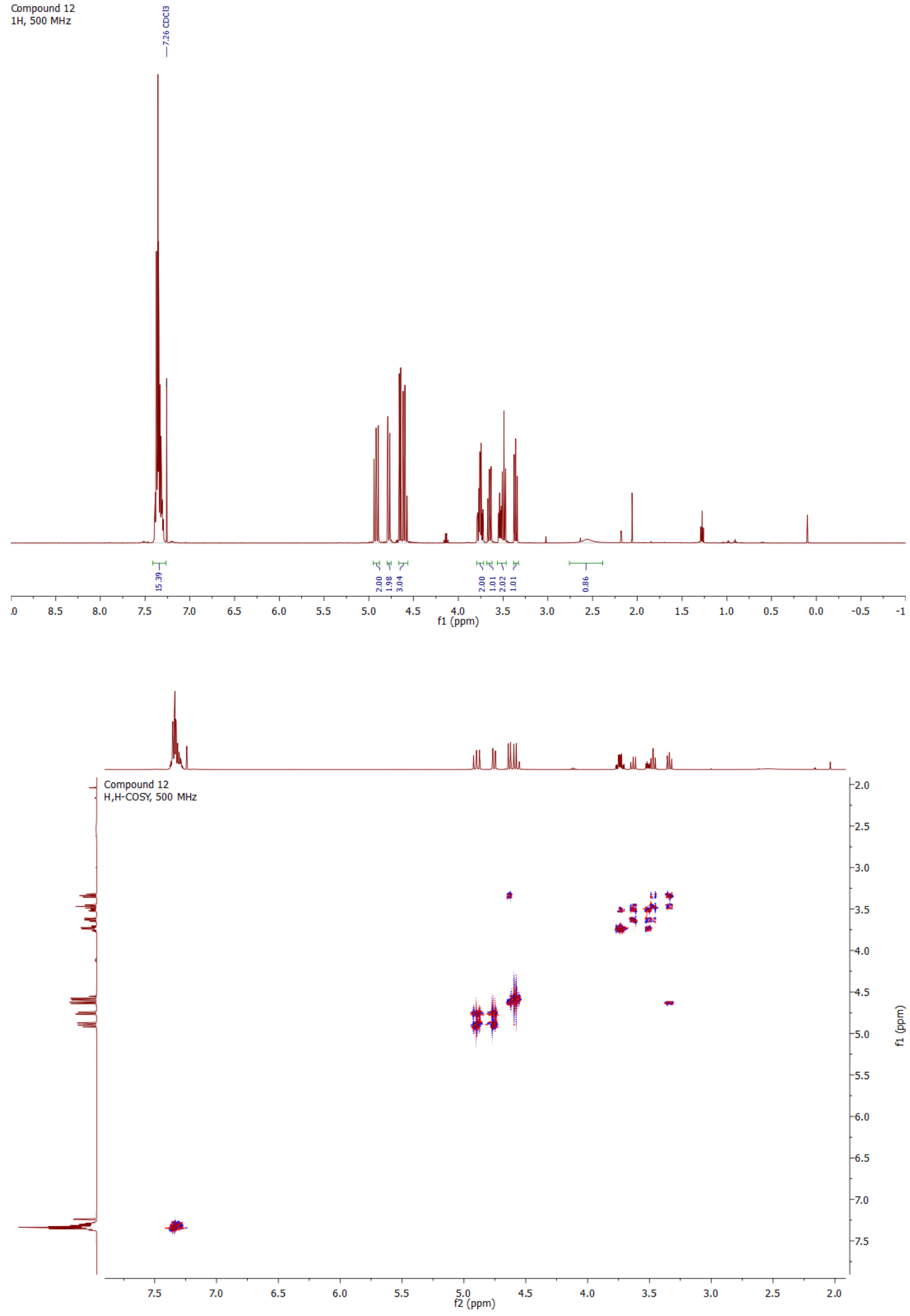


\section{References}

1 L. Su, Y. Zhao, G. Chen and M. Jiang, Polym. Chem., 2012, 3, 1560.

2 A. Pathigoolla and K. M. Sureshan, Chem. Commun., 2014, 50, 317-319.

3 M. E. Evans, Carbohydr. Res., 1972, 21, 473-475. 


\section{Manuskript 3}

Synthesis of block copolymers containing triazole-linked pseudo-cellulose blocks via Huisgen Cycloaddition

Pascal Fuchs ${ }^{\dagger}$, Philipp Vana ${ }^{\ddagger}$ and Kai Zhang ${ }^{+*}$

+ Wood Technology and Wood Chemistry, University of Goettingen, Buesgenweg 4, 37077 Goettingen, Germany

¥ Institute of Physical Chemistry, University of Goettingen, Tammannstr. 6, 37077 Goettingen, Germany

* Corresponding author. E-mail: kai.zhang@uni-goettingen.de 


\section{Abstract}

Block copolymers with the blocks containing carbohydrates are still rare. Herein, the glucose-derived monomer 2,3,6-tris-O-benzyl-4-O-propargyl- $\beta$-D-glucopyranosyl azide was polymerized at endmodified polyethylene glycol (PEG) using the Copper-Catalyzed Azide-Alkyne Cycloaddition (CuAAC). By varying the amount of dosed monomer, different linear diblock-copolymers pseudo-cellulose-blockPEG and triblock-copolymers pseudo-cellulose-block-PEG-block-pseudo-cellulose were obtained. The polymerization reactions expired with nearly complete consumption of the monomer and high yields between $88-94 \%$ were achieved. The dispersity $\Theta$ of obtained block copolymers was measured to be between 2.4 and 2.5. Obtained polymers showed amphiphilic properties that helped to fractionate the polymers into lower and higher molecular weight fractions and thereby narrowed the dispersity $\theta$ to around 1.5. Furthermore, the lower molecular weight fractions could self-assembly to spherical nanoparticles in a 1:1 (v/v) water/THF mixture. This polymerization procedure further expands the scope for the synthesis of diverse saccharide-based block copolymers with various functional groups and applications.

\section{Introduction}

Finding new polymerization techniques and new monomers are subjects of ongoing interest in polymer research. ${ }^{1}$ Many polymerization reactions have been developed from reactions that are used for the transformation of small molecules. For instance, click reactions reported by SHARPLESS ET AL. in 2001 are one efficient group of them. ${ }^{2}$ These reactions have many advantages, such as mild reaction conditions, high efficiency, regioselectivity and simple product isolation. One of the most known click reactions is the Huisgen cycloaddition as copper-catalyzed azide-alkyne cycloaddition (CuAAc). Compared to its abundant applications for the postmodification of polymers and in carbohydrate chemistry ${ }^{3}$, it found less attention as polymerization technique. By using either $A_{2} / B_{2}$ type monomers or $A B$ type monomers, linear polymers were obtained via CUAAC, but their dispersity $\oplus$ was commonly higher than the polymers obtained using living polymerization techniques. ${ }^{4-6}$

In carbohydrate chemistry, the CuAAC of bifunctional mono- or oligosaccharides predominantly led to the formation macrocycles. ${ }^{7}$ Reports about linear triazole-linked saccharide-based polymers are scarce. One example is the topochemical polymerization of a bifunctional galactose derivate, which led to 1,5 -triazole-linked polygalactosides. ${ }^{8}$ Moreover, the use of a starter molecule bearing only one azide or alkyne group to prevent the cyclization has proven successful, because the chain growth from the starter molecule did not have a second click-reactive end group. ${ }^{9}$ Such a starter molecule can also be an end-modified polymer, which allows the synthesis of block copolymers with polysaccharide- 
blocks. Hereby, mostly enzymatic methods were used to polymerize monosaccharides at the end of conventional polymers, such as polystyrene or poly(ethylene glycol). ${ }^{10}$ In comparison, direct polymerization of modified monosaccharides gives perfect control over the regioselectivity of newly introduced groups for the introduction of new functionalities into the polysaccharide blocks. However, the choice of monosaccharides for an artificial enzymatic polymerization is limited and modified monosaccharides even may not be polymerizable by enzymes. Therefore, alternative polymerization approaches have been proposed for the polymerization of modified monosaccharides, such as the ring opening polymerization for 4,6-carbonate-linked polyglucoside. ${ }^{11,12}$ Such methods allowed the synthesis of block-copolymers by successively adding different monomers, in order to get different functions and properties.

Thus, in this work, we report the novel click-polymerization of a bifunctional glucose-derived monomer via CUAAC at the end groups of PEG. Hereby, linear amphiphilic block-copolymers with one block of the conventional polymer PEG and novel pseudo-saccharide blocks were obtained. Moreover, different solubilities of the two types of blocks can be used for the fractionation of block copolymers by chain length or for the formation of spherical nanoparticles.

\section{Results and Discussion}

For the monomer synthesis, glucose was peracetylated at first. Then, a bromine moiety was introduced at $\mathrm{C} 1$ as $\alpha$ anomer and substituted subsequently by azide moieties as $\beta$ anomer. Thereafter, the glucose derivative was deacetylated, $\mathrm{C} 4$ and $\mathrm{C} 6$ positions were protected as benzaldehyde acetal, while $\mathrm{C} 2$ and C3 positions were etherified with benzyl groups. A reductive cleavage of the acetal gave a free hydroxyl group at C4, which was further etherified with propargyl bromide. Finally, 2,3,6-tris-0-benzyl-4-0propargyl- $\beta$-D-glucopyranosyl azide $\mathbf{3}$ was obtained (Scheme 1). For further synthesis of linear polymers, starting molecules with either azide or alkyne moieties need to be present. PEG 2000 has been chosen as a polymeric starting molecule. It has an average degree of polymerization (DP) of around 45 and hydroxyl end groups, which can be conveniently converted through an esterification or etherification reaction to various end groups. The excellent solubility of PEG 2000 in a large variety of polar and nonpolar solvents is also an advantage, which range from water over methanol, ethanol to THF, chloroform, methylene chloride and toluene. In this report, alkyne groups were introduced into the ends of PEG by etherification of the hydroxyl groups with propargyl bromide. Hereby, the etherification of monomethoxypolyethylene glycol 2000 (1a) gave PEG terminated with one propargyl group (2a), while the etherification of PEG 2000 (2b) introduced two propargyl end groups. Due to the linear structure of $\mathbf{2} \mathbf{a}$ and $\mathbf{2} \mathbf{b}$, it can be assumed that the alkyne end groups are not sterically hindered and therefore well accessible by the monomers. The CuAAC-polymerization of the glucose-based 
monomer $\mathbf{3}$ was performed in THF, which is a good solvent for the PEG derivatives as well as for the monomer 3. A triphenylphosphine-free catalyst system copper(I)-iodide/diisopropylethylamine was chosen to avoid the Staudinger reaction between the triphenylphosphine and azide groups, which led to a comparatively lower DPs. ${ }^{6}$ The reactions were performed with either 50 or 80 equivalents of monomer. In contrast to the polysaccharides with diverse glycosidic bonds, such as $\beta-1,4-$ glycosidic bonds within linear cellulose chains, the glucose-based monomers are connected via $\beta$-1,4-triazole linkages in obtained linear copolymers. Thus, these saccharide blocks can be considered to have a pseudo-cellulose structure. Finally, the diblock-copolymers pseudo-cellulose-block-PEG $\left(\mathbf{D}_{\mathbf{5 0}}\right.$ and $\mathbf{D}_{\mathbf{8 0}}$, Scheme 1) and triblock-copolymers pseudo-cellulose-block-PEG-block-pseudo-cellulose ( $\mathbf{T}_{\mathbf{5 0}}$ and $\left.\mathbf{T}_{\mathbf{8 0}}\right)$ were obtained.
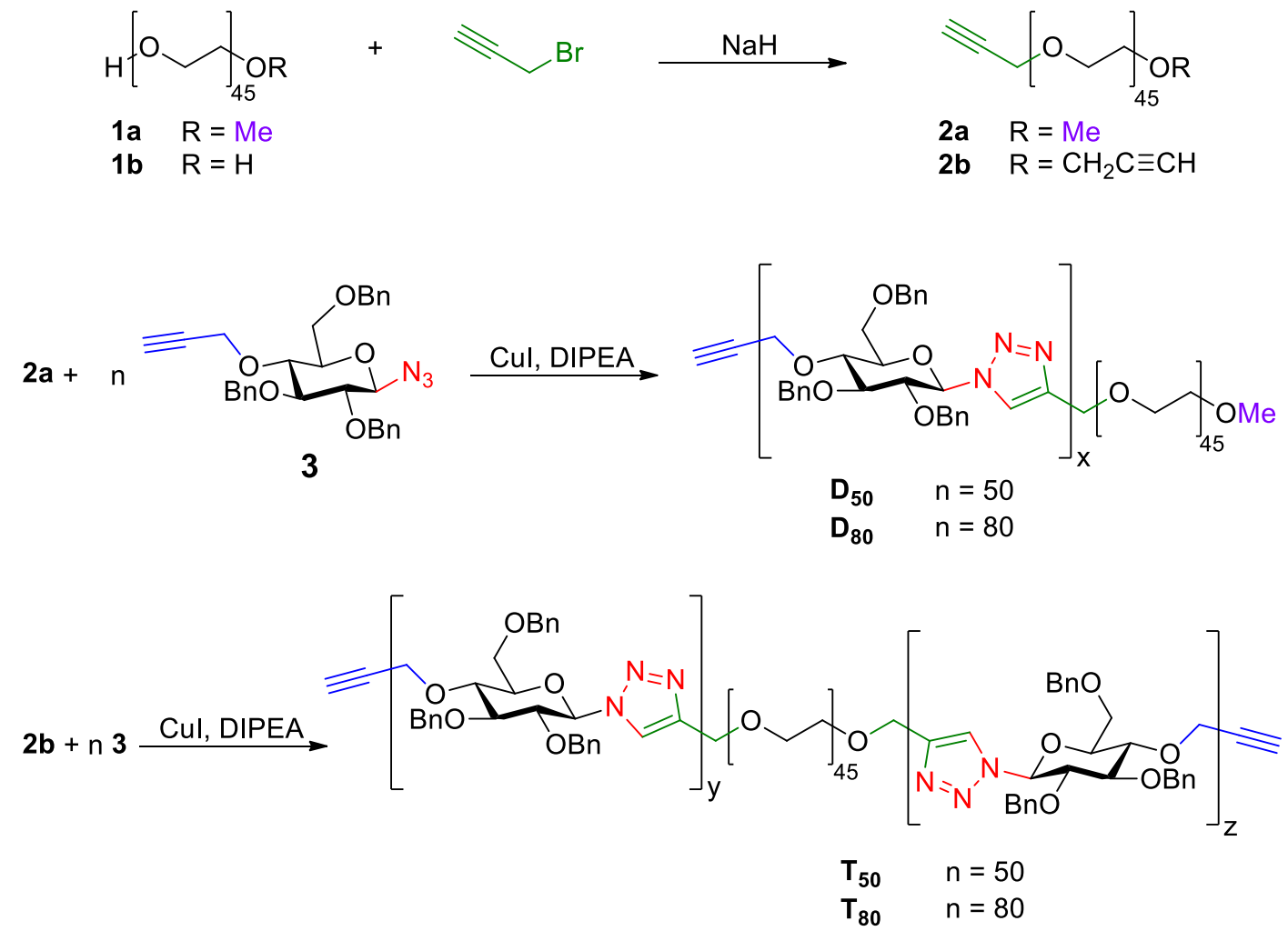

Scheme 1. Schematic illustration for the end group modification of methoxy-terminated PEG 2000 (1a) and PEG 2000 (1b), leading to PEG 2000 with one (2a) or two (2b) propargyl end groups. Further polymerization of $\mathbf{2 a}$ and $\mathbf{2 b}$ with glucose-derived monomer $\mathbf{3}$ led to diblock-copolymers $\mathbf{D}_{\mathbf{5 0}}$ and $\mathbf{D}_{\mathbf{8 0}}$ and triblock-copolymers $\mathbf{T}_{\mathbf{5 0}}$ and $\mathbf{T}_{\mathbf{8 0}}$, respectively.

The success of the reaction was proven by NMR spectroscopy. The chemical shifts in the NMR spectra of all four polymers are very similar and mainly differ in the intensity of the PEG signals. For the signal assignment, ${ }^{1} \mathrm{H}-\mathrm{NMR},{ }^{1} \mathrm{H},{ }^{1} \mathrm{H}$-COSY and HSQC spectra were recorded and evaluated. The ${ }^{13} \mathrm{C}-\mathrm{NMR}$ 
spectrum of $\mathbf{D}_{80}$ is exemplarily shown in Figure 1a. C1 gives the most downfield-shifted signal and C6 gives the most highfield-shifted signal of carbon atoms from the glucose ring. Compared to $\mathrm{C} 1$ of many polysaccharides, such as cellulose, $\mathrm{C} 1$ is not connected to the oxygen atom of the glycosidic bond, but directly with the triazole ring, which causes the highfield-shift of $\mathrm{C} 1$ signal. The signals of the methylene groups could not be precisely assigned, because no ${ }^{4} \mathrm{~J}$ couplings over the oxygen atoms at $\mathrm{C} 2, \mathrm{C} 3, \mathrm{C} 4$, C6 and over C9 were observed. For the triazole-carbon atoms C8 and C9, only one set of signals is obtained. Therefore, it can be assumed that the CuAAC polymerization occurred regioselectively. Commonly, a 1,4-triazole linkage is obtained after the CUAAC.
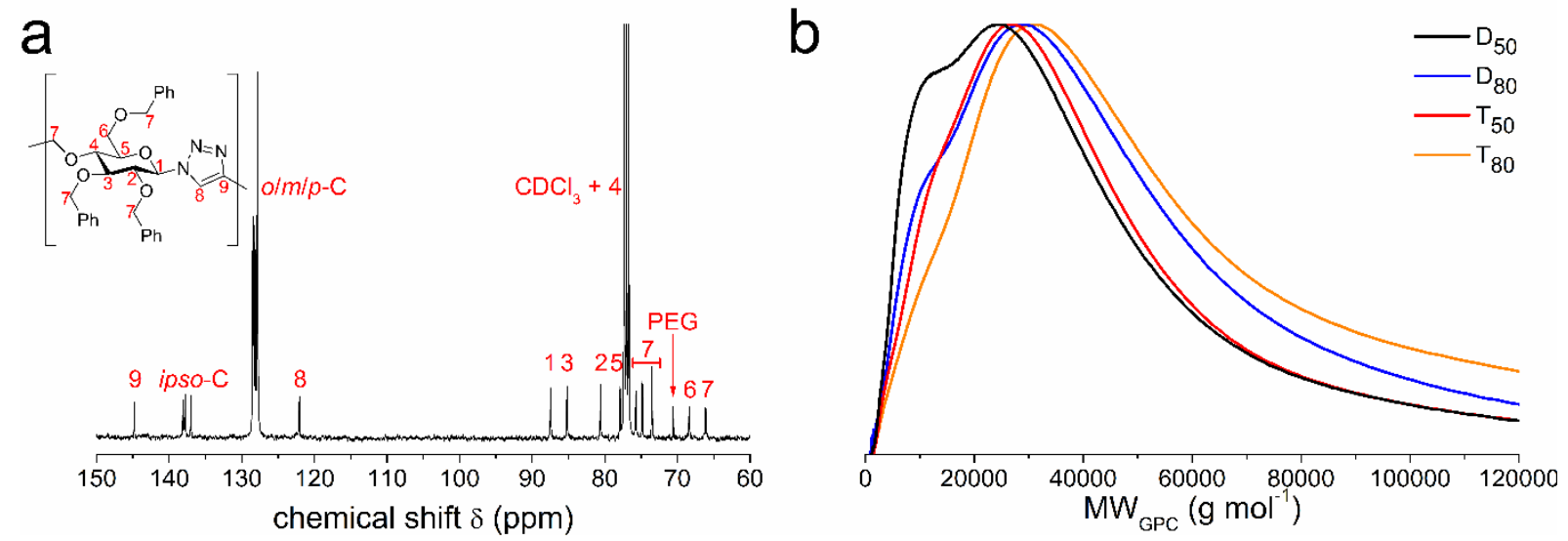

Figure 1a. ${ }^{13} \mathrm{C}-\mathrm{NMR}$ spectrum of $\mathbf{D}_{\mathbf{8 0}}$. b. GPC curves of $\mathbf{D}_{50}, \mathbf{D}_{\mathbf{8 0}}, \mathbf{T}_{50}$ and $\mathbf{T}_{\mathbf{8 0}}$

The yield and consumption of the monomer was nearly quantitative for all click reactions. The degree of polymerization $\left(D P_{n}\right)$ of both diblock-copolymers $\left(D_{50}\right.$ and $\left.D_{80}\right)$ was calculated to be 48 for $D_{50}$ and 75 for $D_{80}$ based on their ${ }^{1} H$ NMR spectra. Hereby, the signal of the methoxy end group of the PEG block was compared to $\mathrm{H} 1$ of the pseudo-cellulose blocks. For the triblock-copolymers $\mathbf{T}_{\mathbf{5 0}}$ and $\mathbf{T}_{\mathbf{8 0}}$, the DP could not be calculated using their ${ }^{1} \mathrm{H}-\mathrm{NMR}$ spectra due to overlap of signals attributed to protons of PEG and pseudo-cellulose blocks, in particular those of $\mathrm{H} 5, \mathrm{H} 6 \mathrm{a}$ and H6b. Using the signals of the propargyl end groups for the determination of the DP leads to an overestimation of the DP, which indicates the partial decomposition of propargyl end groups during or after the polymerization reaction. Due to this chain termination, a wider size distribution of the resulting block-copolymer chains can be expected.

To get a more detailed picture of the chain length distribution of the block-copolymers, size exclusion chromatography (SEC) was performed with polystyrene standards for calibration (Figure 1b). A number-average molecular weight $M_{n}=11.5 \mathrm{~kg} \mathrm{~mol}^{-1}$ with a dispersity $Ð=2.12$ was measured for $D_{50}$ and $\mathrm{M}_{\mathrm{n}}=12.4 \mathrm{~kg} \mathrm{~mol}^{-1}$ with a slightly higher dispersity $Ð=2.48$ for $\mathbf{D}_{\mathbf{8 0}}$ (Table 1 ). For both diblock- 
polymers a shoulder can be observed in the SEC curves at around $11 \mathrm{~kg} \mathrm{~mol}^{-1}$ (Figure $1 \mathrm{~b}$ ). The triblockcopolymer $T_{50}$ was synthesized with $M_{n}=13.9 \mathrm{~kg} \mathrm{~mol}^{-1}$ and dispersity $Ð=2.05$, while $T_{80}$ was even larger with $\mathrm{M}_{\mathrm{n}}=16.5$ and dispersity $Ð=2.39$.

Table 1. SEC results, experimental yield and $D P_{n}$.

\begin{tabular}{clllll}
\hline Sample & $\mathrm{M}^{\mathrm{a}}\left(\mathrm{kg} \mathrm{mol}^{-1}\right)$ & $\mathrm{M}_{\mathrm{w}}{ }^{\mathrm{a}}\left(\mathrm{kg} \mathrm{mol}^{-1}\right)$ & $\oplus\left(\mathrm{M}_{\mathrm{w}} / \mathrm{M}_{\mathrm{n}}\right)$ & Yield (\%) & $\mathrm{DP}^{\mathrm{b}}$ \\
\hline $\mathrm{D}_{50}$ & 11.5 & 24.4 & 2.12 & 94 & 48 \\
$\mathrm{D}_{50 \mathrm{~L}}$ & 8.3 & 12.3 & 1.49 & 34 & 27 \\
$\mathrm{D}_{50 \mathrm{H}}$ & 30.4 & 42.8 & 1.41 & 55 & 148 \\
$\mathrm{D}_{80}$ & 12.4 & 30.6 & 2.48 & 90 & 75 \\
$\mathrm{D}_{80 \mathrm{~L}}$ & 6.5 & 9.2 & 1.41 & 25 & 21 \\
$\mathrm{D}_{80 \mathrm{H}}$ & 24.0 & 40.3 & 1.68 & 68 & 123 \\
$\mathrm{~T}_{50}$ & 13.9 & 28.6 & 2.05 & 93 & n.p. \\
$\mathrm{T}_{50 \mathrm{~L}}$ & 8.8 & 12.7 & 1.45 & 25 & n.p. \\
$\mathrm{T}_{50 \mathrm{H}}$ & 29.4 & 41.3 & 1.41 & 65 & n.p. \\
$\mathrm{T}_{80}$ & 16.5 & 39.4 & 2.39 & 88 & n.p. \\
$\mathrm{T}_{80 \mathrm{~L}}$ & 6.3 & 9.0 & 1.43 & 11 & n.p. \\
$\mathrm{T}_{80 \mathrm{H}}$ & 22.1 & 41.6 & 1.88 & 69 & n.p.
\end{tabular}

a $\overline{\text { measured via SEC in THF relative to polystyrene standards. }{ }^{b} \text { calculated from NMR spectra. }{ }^{c} \text { n.p. }=}$ not possible

The obtained block-copolymers are amphiphiles, consisting of a hydrophilic PEG-block and one or two hydrophobic pseudo-cellulose blocks in one polymer chain. This structural feature and the different solubility of two types of blocks in distinct organic solvents can be used for the fractionation of these copolymers. By dissolving the crude polymers $\left(\mathbf{D}_{\mathbf{5 0}}, \mathbf{D}_{\mathbf{8 0}}, \mathbf{T}_{\mathbf{5 0}}\right.$ and $\left.\mathbf{T}_{\mathbf{8 0}}\right)$ in THF and adjusting the amount of methanol added to the solution, precipitation was observed. Compared to the pseudo-cellulose, PEG is well soluble in methanol. As the proof of concept, the polymers were only separated herein in two fractions. The precipitated fraction mostly consists of polymers with longer pseudo-cellulose chains, which means they have a higher molecular weight $\left(\mathbf{D}_{50 \mathrm{H}}, \mathbf{D}_{\mathbf{8 0 H}}, \mathbf{T}_{\mathbf{5 0 H}}, \mathbf{T}_{\mathbf{8 0 H}}\right.$, Table 1). The $D P_{n}$ obtained from NMR measurements of both diblock-copolymers is between 123 and 148 . SEC measurements revealed a $M_{n}=22.1-30.4 \mathrm{~kg} \mathrm{~mol}^{-1}$ for all precipitated polymer fractions, while their dispersity $Ð$ was narrowed to 1.41-1.88. In addition, $\mathbf{D}_{\mathbf{8 O H}}$ and $\mathbf{T}_{\mathbf{8 O H}}$ were obtained in higher yields compared to their counterparts from the polymerization with less equivalents of monomer $\left(D_{50 \mathrm{H}}\right.$ and $\left.\mathbf{T}_{\mathbf{5 0 H}}\right)$. Polymer chains with a lower molecular weight stayed dissolved ( $\mathbf{D}_{50 \mathrm{~L}}, \mathbf{D}_{\mathbf{8 0 L}}, \mathbf{T}_{\mathbf{5 0 L}}, \mathbf{T}_{\mathbf{8 0 L}}$, Table 1 ) and 
were collected by the evaporation of solvents. They are considerably shorter with a $M_{n}$ between 6.3$8.8 \mathrm{~kg} \mathrm{~mol}^{-1}$ and dispersity $Ð$ of 1.41-1.49. Moreover, the $\mathrm{DP}_{\mathrm{n}}$ of the diblock-copolymers is between 21 and 27.

The low molecular weight fractions $\left(D_{50 L}, D_{80 L}, T_{50 L}, T_{80 L}\right)$ have been used for self-assembly experiments. When precipitating a $5 \mathrm{mg} \mathrm{ml}^{-1}$ THF solution of the polymeric samples in a water/THF mixture and adjusting the final concentration to $0.5 \mathrm{mg} \mathrm{ml}^{-1}$ in $1: 1(\mathrm{v} / \mathrm{v})$ water/THF mixture, spherical nanoparticles were obtained. Their size ranges from $88 \pm 20 \mathrm{~nm}\left(\mathbf{D}_{50 \mathrm{~L}}\right)$ over $195 \pm 91 \mathrm{~nm}\left(\mathbf{D}_{80 \mathrm{~L}}\right)$ and $213 \pm 74 \mathrm{~nm}\left(\mathbf{T}_{80 \mathrm{~L}}\right)$ to $243 \pm 63 \mathrm{~nm}\left(T_{50 L}\right)$. Furthermore, the nanoparticles formed from $D_{50 L}$ and $T_{50 L}$ sedimented after short term, long-time stable nanoparticle dispersions were obtained for $\mathbf{D}_{\mathbf{8 0}}$ and $\mathbf{T}_{\mathbf{8 0}}$.

\section{Conclusion}

In brief, we showed a novel method to synthesize block copolymers with a PEG block and pseudocellulose blocks via Huisgen cycloaddition. Due to the amphiphilic properties of the copolymers, the fractionation to a lower molecular weight fraction and higher molecular weight fractions with much narrower dispersity $Ð$ of polymers was possible by using their different solubilities. In general, the procedure in this report could be applied to monomers derived from various saccharides and diverse end-functionalized conventional polymers. It further expands the scope for the synthesis of diverse saccharide-based block copolymers with various functional groups and applications.

\section{Acknowledgements}

We thank German Research Foundation (DFG) for the project with the project number ZH546/3-1 for the financial support. Furthermore, we thank Sandra Lotze and Katharina Hendrich for the SEC measurements as well as Huan Liu for SEM measurements.

\section{Supplementary material}

The experimental section, SEM pictures, ESI-MS data of the end-modified PEGs (2a and $\mathbf{2} \mathbf{b}$ ), ${ }^{1} \mathrm{H}-,{ }^{1} \mathrm{H},{ }^{1} \mathrm{H}-$ COSY- and HSQC-NMR-spectra as well as all SEC curves are available as supplementary material in one file (file type: .docx).

\section{References}

(1) Qin, A.; Lam, J. W. Y.; Tang, B. Z. Click Polymerization. Macromolecules 2010, 43, 8693-8702.

(2) Kolb, H. C.; Finn, M. G.; Sharpless, K. B. Click Chemistry. Angew. Chem., Int. Ed. 2001, 40, 20042021. 
(3) Tiwari, V. K.; Mishra, B. B.; Mishra, K. B.; Mishra, N.; Singh, A. S.; Chen, X. Cu-Catalyzed Click Reaction in Carbohydrate Chemistry. Chem. Rev. 2016, 116, 3086-3240.

(4) van Steenis, D. J. V. C.; David, O. R. P.; van Strijdonck, G. P. F.; van Maarseveen, J. H.; Reek, J. N. H. Click-chemistry as an efficient synthetic tool for the preparation of novel conjugated polymers. Chem. Commun. [Online] 2005, No. 34, 4333-4335.

(5) Chen, Y.; Guan, Z. Bioinspired modular synthesis of elastin-mimic polymers to probe the mechanism of elastin elasticity. J. Am. Chem. Soc. 2010, 132, 4577-4579.

(6) Binauld, S.; Fleury, E.; Drockenmuller, E. Solving the loss of orthogonality during the polyaddition of $\alpha$-azide- $\omega$-alkyne monomers catalyzed by $\mathrm{Cu}(\mathrm{PPh} 3) 3 \mathrm{Br}$ : Application to the synthesis of high-molar mass polytriazoles. J. Polym. Sci., Part A: Polym. Chem. 2010, 48, 2470-2476.

(7) Kushwaha, D.; Dwivedi, P.; K. Kuanar, S.; K. Tiwari, V. Click Reaction in Carbohydrate Chemistry: Recent Developments and Future Perspective. Curr. Org. Synth. 2013, 10, 90-135.

(8) Pathigoolla, A.; Gonnade, R. G.; Sureshan, K. M. Topochemical Click Reaction. Angew. Chem., Int. Ed. 2012, 124, 4438-4442.

(9) Schmidt, M. S.; Götz, K. H.; Koch, W.; Grimm, T.; Ringwald, M. Studies toward the synthesis of linear triazole linked pseudo oligosaccharides and the use of ferrocene as analytical probe. Carbohydr. Res. 2016, 425, 28-34.

(10) Schatz, C.; Lecommandoux, S. Polysaccharide-containing block copolymers: synthesis, properties and applications of an emerging family of glycoconjugates. Macromol. Rapid Commun. 2010, 31, 16641684.

(11) Su, L.; Khan, S.; Fan, J.; Lin, Y.-N.; Wang, H.; Gustafson, T. P.; Zhang, F.; Wooley, K. L. Functional sugar-based polymers and nanostructures comprised of degradable poly(d -glucose carbonate)s. Polym. Chem. 2017, 8, 1699-1707.

(12) Lin, Y.-N.; Su, L.; Smolen, J.; Li, R.; Song, Y.; Wang, H.; Dong, M.; Wooley, K. L. Co-assembly of sugar-based amphiphilic block polymers to achieve nanoparticles with tunable morphology, size, surface charge, and acid-responsive behavior. Mater. Chem. Front. 2018, 2, 2230-2238.

(13) Kantaria, T.; Kantaria, T.; Titvinidze, G.; Otinashvili, G.; Kupatadze, N.; Zavradashvili, N.; Tugushi, D.; Katsarava, R. New 1,2,3-Triazole Containing Polyesters via Click Step-Growth Polymerization and Nanoparticles Made of Them. Int. J. Polym. Sci. 2018, 2018, 1-14.

(14) Miladi, I. A.; Mudraboyina, B. P.; Oueslati, A.; Drockenmuller, E.; Romdhane, H. B. Photochemical isomerization of norbornadiene-containing polytriazoles obtained by click chemistry polyaddition. J. Polym. Sci. Part A: Polym. Chem. 2014, 52, 223-231. 


\section{Manuskript 3 (Supplementary material)}

\section{Synthesis of block copolymers containing triazole-linked pseudo-cellulose blocks via Huisgen Cycloaddition}

Pascal Fuchs, Philipp Vana and Kai Zhang*

\section{Materials}

Toluene and tetrahydrofurane (THF) from Th. Geyer Ingredients GmbH were dried over sodium/benzophenone and distilled prior to use. Propargyl bromide from Alfa aesar, diethyl ether from AppliChem $\mathrm{GmbH}, \mathrm{N}, \mathrm{N}$-diisopropylethylamine (DIPEA), polyethylene glycol (PEG) 2000 and copper(I)-iodide from Carl Roth $\mathrm{GmbH}$, polyethylene glycol 2000 monomethyl ether from Merck KGaA, dichloromethane, sodium hydride (60 wt\% dispersion in mineral oil) and methanol from Th. Geyer Ingredients $\mathrm{GmbH}$ were used as received.

\section{Methods}

${ }^{1} \mathrm{H}-\mathrm{NMR}$ spectra, ${ }^{1} \mathrm{H},{ }^{1} \mathrm{H}$-COSY spectra, liquid-state ${ }^{13} \mathrm{C}-\mathrm{NMR}$ and HSQC spectra were obtained on a Bruker $300 \mathrm{MHz}$ NMR spectrometer or a Bruker Avance Neo $400 \mathrm{MHz}$ in $\mathrm{CDCl}_{3}$ at $25^{\circ} \mathrm{C}$. Up to 32 scans were accumulated for the ${ }^{1} \mathrm{H}-\mathrm{NMR}$ spectra. Scans of up to 512 were accumulated for the ${ }^{13} \mathrm{C}-\mathrm{NMR}$ spectra. Chemical shifts are reported relative to the solvent peak.

Size exclusion chromatography (SEC) characterization of all samples was performed on an Agilent 1260 Infinity system with THF as the eluent. The system was composed of an autosampler (Agilent 1260 Infinity Standard Autosampler), an isocratic solvent pump (Agilent 1260 Infinity), a precolumn of the type SDV $(8 \mathrm{~mm} \times 50 \mathrm{~mm}$, particle size $10 \mu \mathrm{m})$ from the company Polymer Standard Services (PSS), three separation columns of the type SDV $(8 \mathrm{~mm} \times 300 \mathrm{~mm}$, nominal particle size: $10 \mu \mathrm{m}$, with pore sizes of $10^{6}, 10^{5}$, and $10^{3} \AA$ ) from PSS maintained at $35^{\circ} \mathrm{C}$ in a column compartment and a refractive

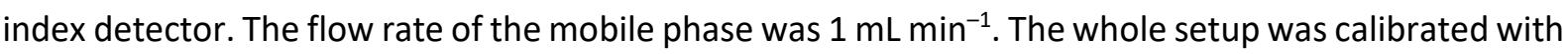
polystyrene standards of low dispersity and toluene as internal standard. All samples were filtered through a PTFE syringe filter with the pore size of $0.45 \mu \mathrm{m}$ before the injection.

ESI mass spectrometry was performed on a maXis from Bruker Daltonik with a TOF detector. 


\section{End group modification of PEG}

\section{Polyethylene glycol monomethyl monopropargyl ether ${ }^{1}$}

Polyethylene glycol 2000 monomethyl ether ( $5 \mathrm{~g}, 2.5 \mathrm{mmol}$ hydroxyl groups) was dissolved in $60 \mathrm{ml}$ toluene at $50^{\circ} \mathrm{C}$. After a clear solution was obtained, it was allowed to cool down to rt and sodium hydride $(7.5 \mathrm{mmol}, 260 \mathrm{mg}$ of $60 \%$ dispersion in mineral oil) was added. After stirring the reaction solution for $1 \mathrm{~h}$ at $\mathrm{rt}$, the gas evolution ceased and the solution turned orange. Propargyl bromide $(836 \mu \mathrm{l}, 7.5 \mathrm{mmol}$ ) was added and the solution was stirred for further $3 \mathrm{~d}$ at rt. The orange color faded. The suspension was filtrated and toluene was distilled off. The obtained oil was dissolved in $5 \mathrm{ml} \mathrm{DCM}$ and precipitated $75 \mathrm{ml}$ diethyl ether three times. The white solid product was dried in vacuo to yield $3.3 \mathrm{~g}$ of the polyethylene glycol monomethyl monopropargyl ether.

${ }^{1} \mathrm{H}$ NMR $\left(300 \mathrm{MHz}\right.$, Chloroform-d) $\delta=4.18\left(\mathrm{~d}, \mathrm{~J}=2.3,2 \mathrm{H}, \mathrm{OCH}_{2} \equiv \mathrm{CH}\right), 3.62(\mathrm{~s}, 200 \mathrm{H}), 3.36\left(\mathrm{~s}, 3 \mathrm{H}, \mathrm{OCH}_{3}\right)$, $2.42(t, J=2.4,1 \mathrm{H}, \equiv C H)$.

Complete conversion of the starting material was confirmed by ESI mass spectroscopy.

\section{Polyethylene glycol dipropargyl ether}

PEG 2000 (5 g, $2.5 \mathrm{mmol}, 5 \mathrm{mmol}$ hydroxyl groups) was dissolved in $60 \mathrm{ml}$ toluene at $50{ }^{\circ} \mathrm{C}$. After a clear solution was obtained, it was allowed to cool down to $\mathrm{rt}$ and sodium hydride $(7.5 \mathrm{mmol}, 260 \mathrm{mg}$ of $60 \%$ dispersion in mineral oil) was added. After stirring the reaction solution for $1 \mathrm{~h}$ at $\mathrm{rt}$, the gas evolution ceased, and the solution turned orange. Propargyl bromide $(836 \mu \mathrm{l}, 7.5 \mathrm{mmol})$ was added and the solution was stirred for further $3 \mathrm{~d}$ at $\mathrm{rt}$. The orange color faded. The suspension was filtrated, and toluene was distilled off. The obtained oil was dissolved in $5 \mathrm{ml} \mathrm{DCM}$ and precipitated in $75 \mathrm{ml}$ diethyl ether. This process was repeated for three times. Resulting white solid product was dried in vacuo to yield $3.25 \mathrm{~g}$ of the polyethylene glycol dipropargyl ether.

${ }^{1} \mathrm{H}$ NMR $\left(300 \mathrm{MHz}, \mathrm{CDCl}_{3}\right) \delta=4.20\left(\mathrm{~d}, \mathrm{~J}=2.4,4 \mathrm{H}, \mathrm{OCH}_{2}=\mathrm{CH}\right), 3.64\left(\mathrm{~s}, 200 \mathrm{H}, \mathrm{O}-\left(\mathrm{CH}_{2}\right)_{2}-\mathrm{O}\right), 2.44(\mathrm{t}, \mathrm{J}=2.4$, $2 \mathrm{H}, \equiv \mathrm{CH})$.

Complete conversion of the starting material was confirmed by ESI mass spectroscopy.

\section{Synthesis of glucose-derived monomer 2,3,6-tris-0-benzyl-4-propargyl- $\beta$-D-glucopyranosyl azide}

First, glucose was peracetylated, a bromine moiety was introduced at C1 in $\alpha$-configuration and it was subsequently nucleophilic substituted by an azide group ( $\beta$-configuration). ${ }^{2}$ The derivative was deacetylated ${ }^{3}$, then $\mathrm{C} 4$ and $\mathrm{C} 6$ were protected as benzaldehyde acetal. ${ }^{4}$ After the protection of $\mathrm{C} 2$ and 
C3 as benzyl ether, the selective reductive cleavage of the acetal gave a free hydroxyl at C4, which was etherified with propargyl bromide in the last step. ${ }^{3}$

\section{Click reaction}

\section{General polymerization procedure}

The polyethylene glycol dipropargyl ether $(22.4 \mathrm{mg}, 22.4 \mu \mathrm{mol})$ or the polyethylene glycol monomethyl monopropargyl ether (44.8 mg, $22.4 \mu \mathrm{mol}$ ) was dissolved in $8 \mathrm{ml} \mathrm{THF}$ and $1.36 \mathrm{ml}$ DIPEA at rt, degassed for $20 \mathrm{~min}$ and the catalyst copper(I)-iodide was added ( $3.81 \mathrm{mg}, 20 \mu \mathrm{mol})$. Then, the freshly prepared 2,3,6-tris-O-benzyl-4-propargyl- $\beta$-D-glucopyranosyl azide (30 wt\% in THF) was added continuously over $48 \mathrm{~h}$ by using a syringe pump (NE-1010 by New Era Pump Systems Inc). After the addition was completed, the dispersion was stirred at $\mathrm{rt}$ for $48 \mathrm{~h}$. The catalyst and other dispersed solid was centrifuged off and the solvent was distilled off. The obtained yellow solid was purified by repeatedly dissolving in $5 \mathrm{ml} \mathrm{DCM}$ and precipitating in $75 \mathrm{ml}$ diethyl ether.

\section{Diblock-copolymer with 50 equivalents of monomer $\left(D_{50}\right)$}

Polyethylene glycol monomethyl monopropargyl ether $(44.8 \mathrm{mg}, 22.4 \mu \mathrm{mol})$ and 2,3,6-tris-O-benzyl4-propargyl- $\beta$-D-glucopyranosyl azide (586 mg, $1141 \mu \mathrm{mol}$ ) were used.

Yield: $593 \mathrm{mg}$

${ }^{1} \mathrm{H}$ NMR (400 MHz, CDCl $): \delta=7.40-6.85(\mathrm{~m}, \mathrm{Ar}-\mathrm{H}), 5.49(\mathrm{~d}, \mathrm{~J}=9.1,1 \mathrm{H}, \mathrm{H}-1), 5.02\left(\mathrm{~d}, \mathrm{~J}=12.0,1 \mathrm{H}, \mathrm{Ar}-\mathrm{C}^{1} \mathrm{H}_{2^{-}}\right.$ a), $4.94\left(\mathrm{~m}, 2 \mathrm{H}, \mathrm{Ar}-\mathrm{C}^{2} \mathrm{H}_{2}\right), 4.77\left(\mathrm{~d}, \mathrm{~J}=11.6,1 \mathrm{H}, \mathrm{Ar}-\mathrm{C}^{1} \mathrm{H}_{2}-\mathrm{b}\right), 4.48\left(\mathrm{~m}, 2 \mathrm{H}, \mathrm{Ar}-\mathrm{C}^{3} \mathrm{H}_{2}\right), 4.46\left(\mathrm{~m}, 1 \mathrm{H}, \mathrm{Ar}-\mathrm{C}^{4} \mathrm{H}_{2}-\mathrm{a}\right)$, $4.07\left(\mathrm{~d}, J=10.5,1 \mathrm{H}, \mathrm{Ar}_{-} \mathrm{C}^{4} \mathrm{H}_{2}-\mathrm{b}\right), 4.01(\mathrm{t}, \mathrm{J}=9.1,1 \mathrm{H}, \mathrm{H}-2), 3.83(\mathrm{~m}, 2 \mathrm{H}, \mathrm{H}-3 \& \mathrm{H}-4), 3.78-3.72(\mathrm{~m}, 2 \mathrm{H}, \mathrm{H}-$ $6 a \& H-6 b), 3.68(s, P E G), 3.64(m, 1 H, H-5)$.

${ }^{13} \mathrm{C} \mathrm{NMR}\left(100 \mathrm{MHz}, \mathrm{CDCl}_{3}\right): \delta=144.88$ (triazole-C4), 138.18, 137.89, 137.09 (3C, ipso-C), 128.65-127.88 (15C, ortho-C, meta-C, para-C), 122.18 (triazole-C5), 87.58 (C1), 85.30 (C3), 80.70 (C2), 77.99 (C5), $77.36(\mathrm{C} 4), 75.83\left(\mathrm{Ar}-\mathrm{C}^{2} \mathrm{H}_{2}\right), 74.94\left(\mathrm{Ar}-\mathrm{C}^{4} \mathrm{H}_{2}\right), 73.62\left(\mathrm{Ar}-\mathrm{C}^{3} \mathrm{H}_{2}\right), 70.67(\mathrm{PEG}), 68.48(\mathrm{C} 6), 66.21\left(\mathrm{Ar}-\mathrm{C}^{1} \mathrm{H}_{2}\right)$.

\section{Triblock-copolymer with 50 equivalents of monomer $\left(T_{50}\right)$}

Polyethylene glycol dipropargyl ether (22.4 mg, $22.4 \mu \mathrm{mol})$ and 2,3,6-tris-O-benzyl-4-propargyl- $\beta$-Dglucopyranosyl azide (586 mg, $1141 \mu \mathrm{mol}$ ) were used.

Yield: $566 \mathrm{mg}$ 
${ }^{1} \mathrm{H}$ NMR $\left(400 \mathrm{MHz}, \mathrm{CDCl}_{3}\right): \delta=7.40-6.95(\mathrm{~m}, \mathrm{Ar}-\mathrm{H}), 5.49(\mathrm{~d}, \mathrm{~J}=9.1,1 \mathrm{H}, \mathrm{H}-1), 5.02\left(\mathrm{~d}, \mathrm{~J}=12.0,1 \mathrm{H}, \mathrm{Ar}-\mathrm{C}^{1} \mathrm{H}_{2^{-}}\right.$ a), $4.94\left(\mathrm{~m}, 2 \mathrm{H}, \operatorname{Ar}-\mathrm{C}^{2} \mathrm{H}_{2}\right), 4.77\left(\mathrm{~d}, \mathrm{~J}=11.6,1 \mathrm{H}, \mathrm{Ar}-\mathrm{C}^{1} \mathrm{H}_{2}-\mathrm{b}\right), 4.48\left(\mathrm{~m}, 2 \mathrm{H}, \mathrm{Ar}-\mathrm{C}^{3} \mathrm{H}_{2}\right), 4.46\left(\mathrm{~m}, 1 \mathrm{H}, \mathrm{Ar}-\mathrm{C}^{4} \mathrm{H}_{2}-\mathrm{a}\right)$, $4.07\left(\mathrm{~d}, J=10.5,1 \mathrm{H}, \operatorname{Ar}-\mathrm{C}^{4} \mathrm{H}_{2}-\mathrm{b}\right), 4.01(\mathrm{t}, \mathrm{J}=9.1,1 \mathrm{H}, \mathrm{H}-2), 3.83(\mathrm{~m}, 2 \mathrm{H}, \mathrm{H}-3 \& \mathrm{H}-4), 3.78-3.72(\mathrm{~m}, 2 \mathrm{H}, \mathrm{H}-$ $6 a \& H-6 b), 3.68$ (s, PEG), 3.64 (m, 1H, H-5).

${ }^{13} \mathrm{C} \mathrm{NMR}\left(100 \mathrm{MHz}, \mathrm{CDCl}_{3}\right): \delta=144.88$ (triazole-C4), 138.19, 137.89, 137.09 (3C, ipso-C), 128.65-127.84 (15C, ortho-C, meta-C, para-C), 122.18 (triazole-C5), 87.58 (C1), 85.30 (C3), 80.71 (C2), 78.00 (C5), 77.35 (C4), $75.84\left(\mathrm{Ar}-\mathrm{C}^{2} \mathrm{H}_{2}\right), 74.94\left(\mathrm{Ar}-\mathrm{C}^{4} \mathrm{H}_{2}\right), 73.62\left(\mathrm{Ar}-\mathrm{C}^{3} \mathrm{H}_{2}\right), 70.63(\mathrm{PEG}), 68.48(\mathrm{C} 6), 66.21\left(\mathrm{Ar}-\mathrm{C}^{1} \mathrm{H}_{2}\right)$.

\section{Diblock-copolymer with 80 equivalents of monomer $\left(D_{80}\right)$}

Polyethylene glycol monomethyl monopropargyl ether (44.8 mg, $22.4 \mu \mathrm{mol}$ ) and 2,3,6-tris-O-benzyl4-propargyl- $\beta$-D-glucopyranosyl azide (920 mg, $1791 \mu \mathrm{mol})$ were used.

Yield: $865 \mathrm{mg}$

${ }^{1} \mathrm{H}$ NMR (400 MHz, CDCl 3 ): $\left.\delta=8.10-6.90(\mathrm{~m}, \mathrm{Ar}-\mathrm{H}), 5.48(\mathrm{~d}, \mathrm{~J}=9.1,1 \mathrm{H}, \mathrm{H}-1), 4.98(\mathrm{~m}, 1 \mathrm{H}), \operatorname{Ar}-\mathrm{C}^{1} \mathrm{H}_{2}-\mathrm{a}\right)$, $4.92\left(\mathrm{~m}, 2 \mathrm{H}, \operatorname{Ar}-\mathrm{C}^{2} \mathrm{H}_{2}\right), 4.74\left(\mathrm{~m}, 1 \mathrm{H}, \mathrm{Ar}-\mathrm{C}^{1} \mathrm{H}_{2}-\mathrm{b}\right), 4.48\left(\mathrm{~m}, 2 \mathrm{H}, \operatorname{Ar}-\mathrm{C}^{3} \mathrm{H}_{2}\right), 4.46\left(\mathrm{~m}, 1 \mathrm{H}, \mathrm{Ar}-\mathrm{C}^{4} \mathrm{H}_{2}-\mathrm{a}\right), 4.06(\mathrm{~m}$, $\left.1 \mathrm{H}, \operatorname{Ar}-\mathrm{C}^{4} \mathrm{H}_{2}-\mathrm{b}\right), 3.99(\mathrm{~m}, 1 \mathrm{H}, \mathrm{H}-2), 3.81$ (m, 2H, H-3\&H-4), 3.74-3.68 (m, 2H, H-6a\&H-6b), 3.67 (s, PEG), $3.64(m, 1 H, H-5)$.

${ }^{13} \mathrm{C}$ NMR (100 MHz, CDCl $): \delta=144.89$ (triazole-C4), 138.21-137.11 (3C, ipso-C), 129.89-127.99 (15C, ortho-C, meta-C, para-C), 122.21 (triazole-C5), 87.61 (C1), 85.31 (C3), 80.73 (C2), 78.01 (C5), 77.36 (C4), $75.85\left(\operatorname{Ar}-C^{2} \mathrm{H}_{2}\right), 74.96\left(\operatorname{Ar}-C^{4} \mathrm{H}_{2}\right), 73.64\left(\operatorname{Ar}-C^{3} \mathrm{H}_{2}\right), 70.69$ (PEG), $68.48(\mathrm{C} 6), 66.21\left(\operatorname{Ar}-C^{1} \mathrm{H}_{2}\right)$.

\section{Triblock-copolymer with 80 equivalents of monomer $\left(T_{80}\right)$}

Polyethylene glycol dipropargyl ether (22.4 mg, $22.4 \mu \mathrm{mol}$ ) and 2,3,6-tris-O-benzyl-4-propargyl- $\beta$-Dglucopyranosyl azide (920 mg, $1791 \mu \mathrm{mol})$ were used.

Yield: $827 \mathrm{mg}$

$\left.{ }^{1} \mathrm{H} \mathrm{NMR}\left(400 \mathrm{MHz}, \mathrm{CDCl}_{3}\right): \delta=8.10-6.90(\mathrm{~m}, \mathrm{Ar}-\mathrm{H}), 5.48(\mathrm{~m}, 1 \mathrm{H}, \mathrm{H}-1), 4.97(\mathrm{~m}, 1 \mathrm{H}), \operatorname{Ar}^{-1} \mathrm{H}_{2}-\mathrm{a}\right), 4.89(\mathrm{~m}$, 2H, Ar-C $\left.\mathrm{C}^{2} \mathrm{H}_{2}\right), 4.74\left(\mathrm{~m}, 1 \mathrm{H}, \operatorname{Ar}-\mathrm{C}^{1} \mathrm{H}_{2}-\mathrm{b}\right), 4.48\left(\mathrm{~m}, 2 \mathrm{H}, \operatorname{Ar}-\mathrm{C}^{3} \mathrm{H}_{2}\right), 4.46\left(\mathrm{~m}, 1 \mathrm{H}, \operatorname{Ar}-\mathrm{C}^{4} \mathrm{H}_{2}-\mathrm{a}\right), 4.06(\mathrm{~m}, 1 \mathrm{H}, \mathrm{Ar}-$ $\left.\mathrm{C}^{4} \mathrm{H}_{2}-\mathrm{b}\right), 3.97$ ( $\left.\mathrm{m}, 1 \mathrm{H}, \mathrm{H}-2\right), 3.80$ ( $\left.\mathrm{m}, 2 \mathrm{H}, \mathrm{H}-3 \& \mathrm{H}-4\right), 3.74-3.68$ (m, 2H, H-6a\&H-6b), 3.64 (s, PEG), 3.63 (m, $1 \mathrm{H}, \mathrm{H}-5)$. 
${ }^{13} \mathrm{C} \mathrm{NMR}\left(100 \mathrm{MHz}, \mathrm{CDCl}_{3}\right): \delta=144.77$ (triazole-C4), 138.21-137.06 (3C, ipso-C), 133.74, 129.78-127.95 (15C, ortho-C, meta-C, para-C), 122.21 (triazole-C5), 87.39 (C1), 85.27 (C3), 80.50 (C2), 77.66 (C5), 77.10 (C4), $75.859\left(\mathrm{Ar}-\mathrm{C}^{2} \mathrm{H}_{2}\right), 74.96\left(\mathrm{Ar}-\mathrm{C}^{4} \mathrm{H}_{2}\right), 73.65\left(\mathrm{Ar}-\mathrm{C}^{3} \mathrm{H}_{2}\right), 70.65(\mathrm{PEG}), 68.20(\mathrm{C} 6), 65.83\left(\mathrm{Ar}-\mathrm{C}^{1} \mathrm{H}_{2}\right)$.

\section{Fractionation of the polymers}

The polymers were further separated by dissolving $400 \mathrm{mg}$ of polymers in $40 \mathrm{ml}$ THF and precipitating by adding $75 \mathrm{ml}$ methanol. After stirring for $3 \mathrm{~h}$ at $\mathrm{rt}$, the solid was separated by centrifugation and the supernatant solution was dried in vacuo. Both polymer fractions were purified by repeatedly dissolving the polymers in $3 \mathrm{ml} \mathrm{DCM}$ and precipitating in $30 \mathrm{ml}$ diethyl ether.

\section{Supporting Results}
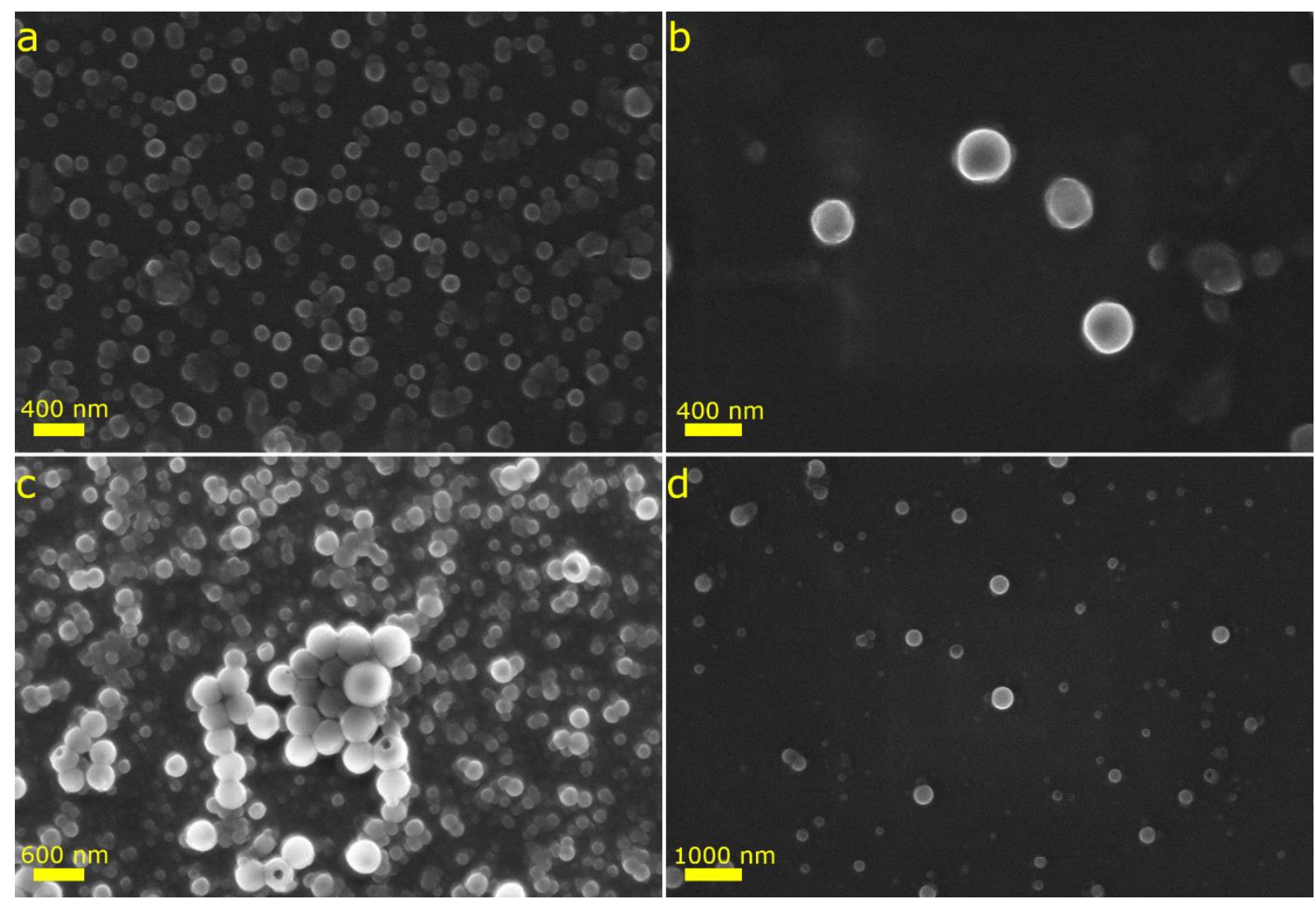

Figure S1. Nanoparticles were formed at $0.5 \mathrm{mg} \mathrm{ml}^{-1}$ in $\mathrm{THF} / \mathrm{H}_{2} \mathrm{O}$ 1:1 (v/v). Concentration was reduced to 0.01

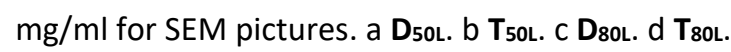




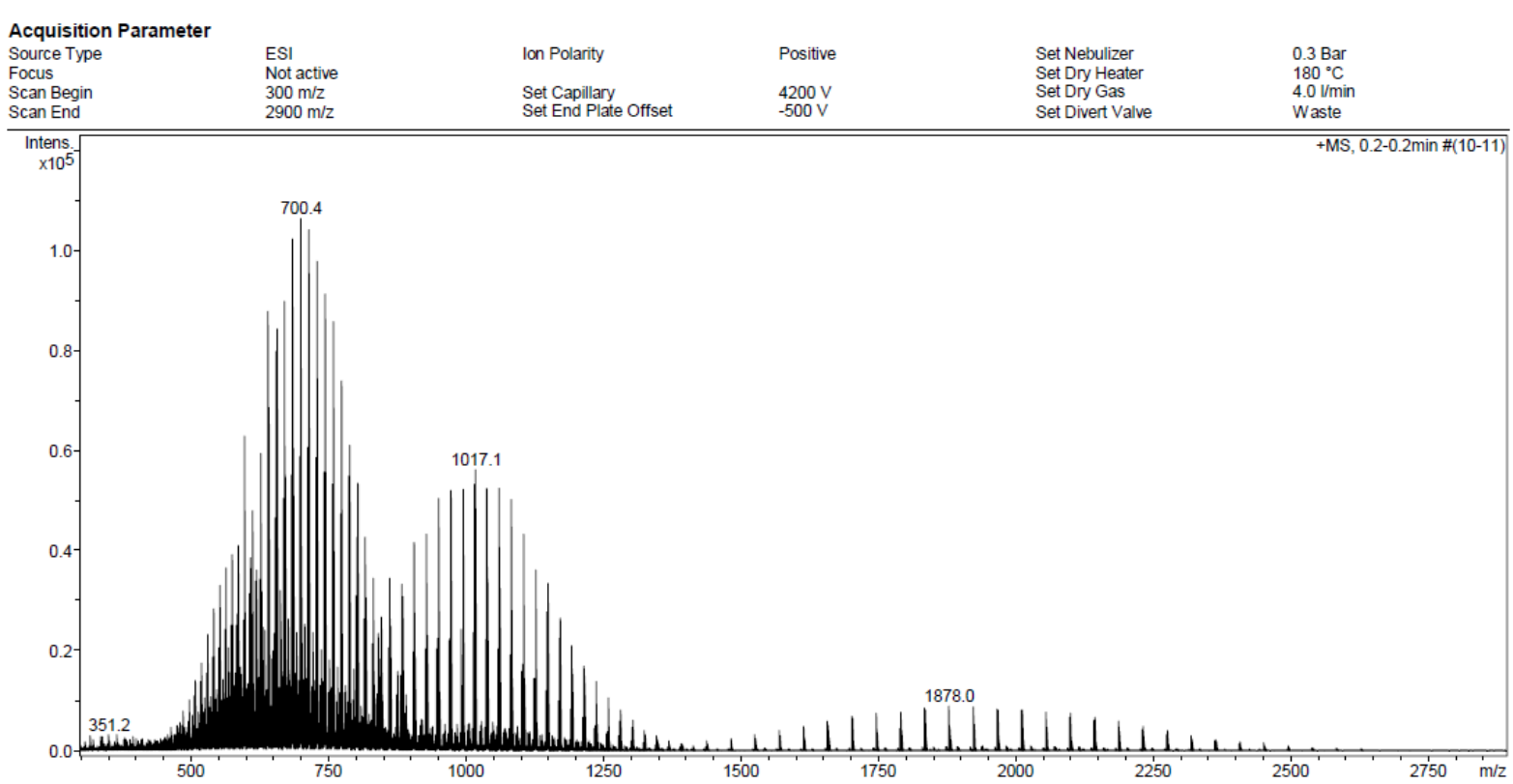

Figure S2. ESI-MS of Polyethylene glycol dipropargyl ether

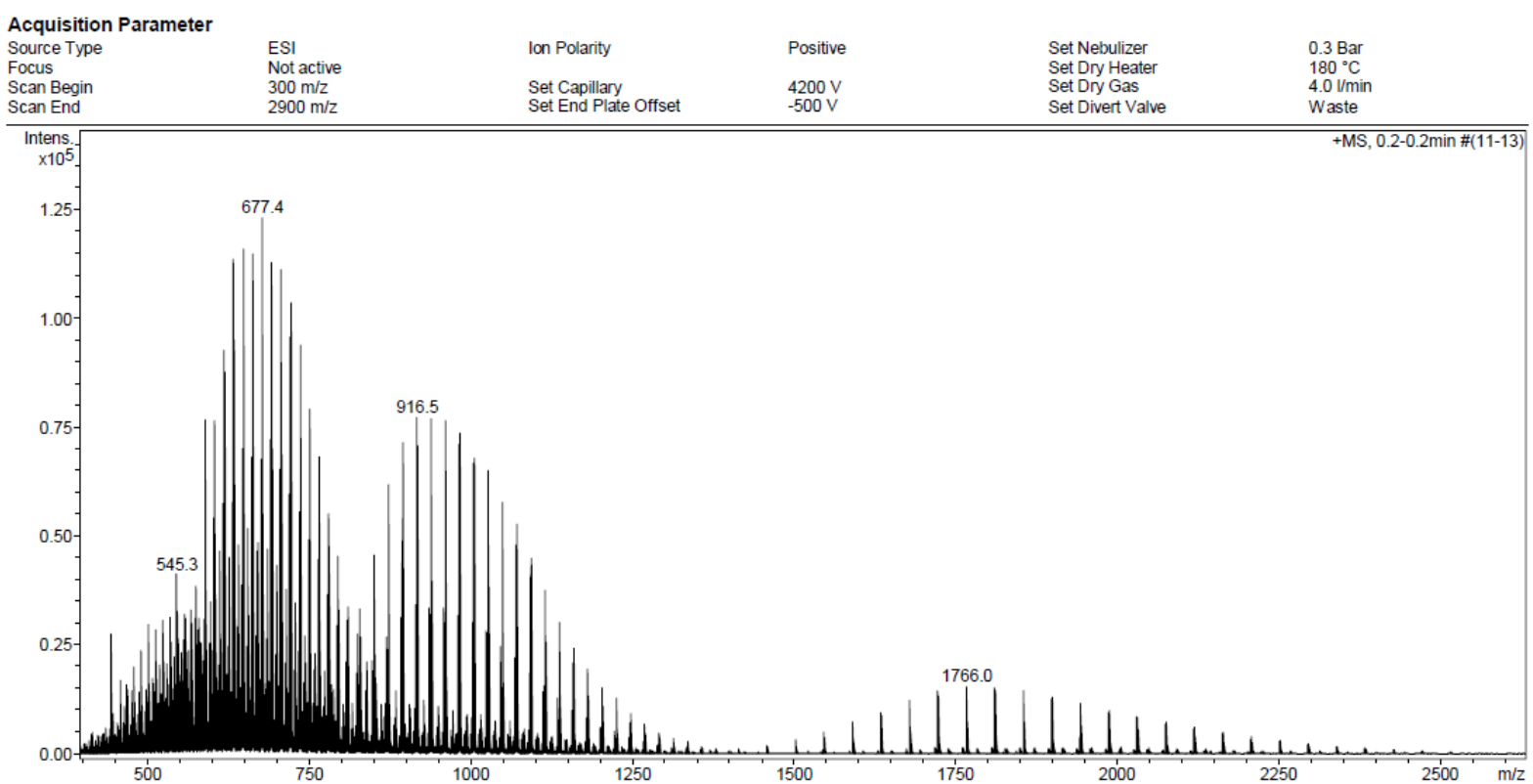

Figure S3. ESI-MS of Polyethylene glycol monomethyl monopropargyl ether 
D80

Proton, $400 \mathrm{MHz}$

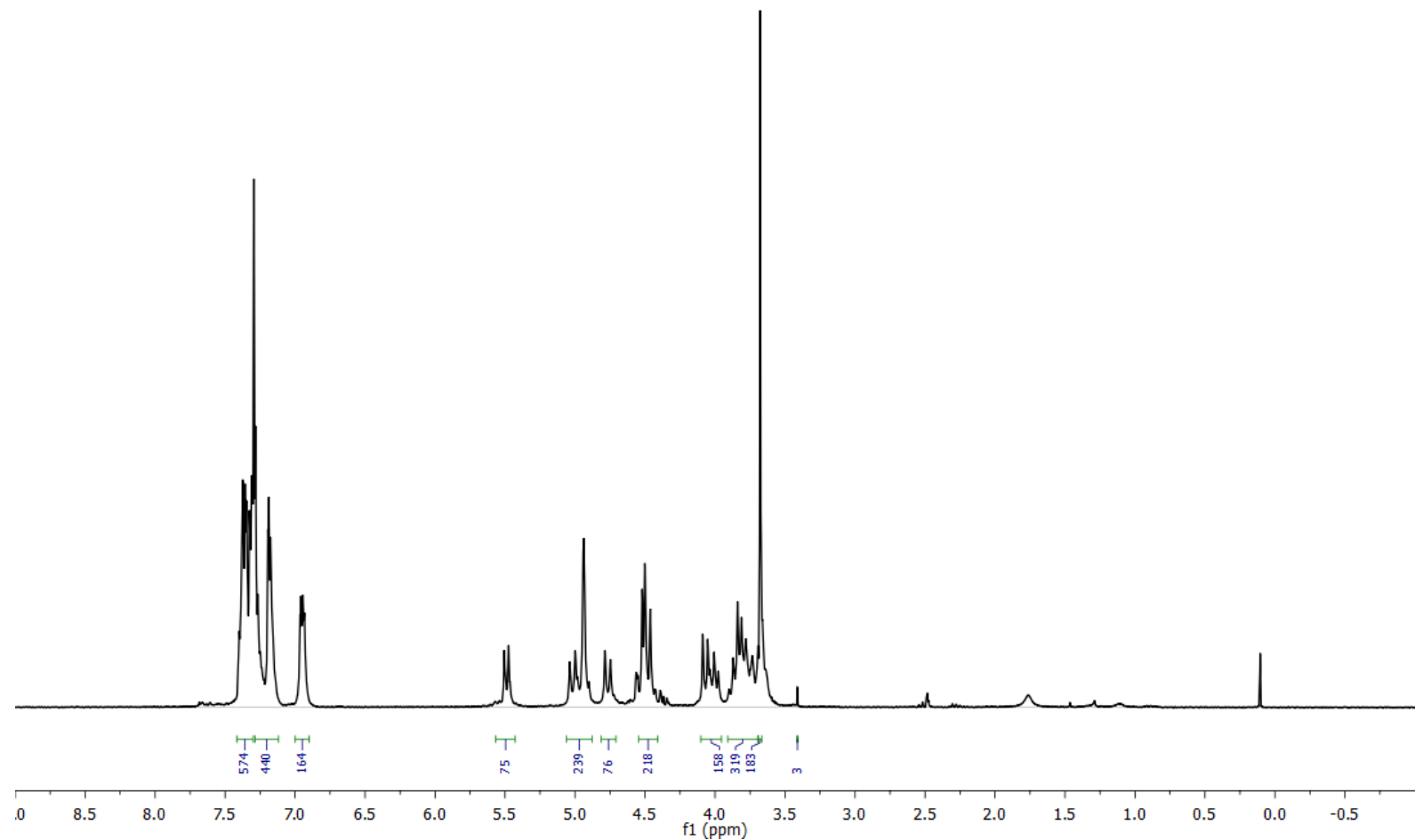

Figure S4. ${ }^{1} \mathrm{H}-\mathrm{NMR}$ spectrum of $\mathbf{D}_{\mathbf{8 0}}$.

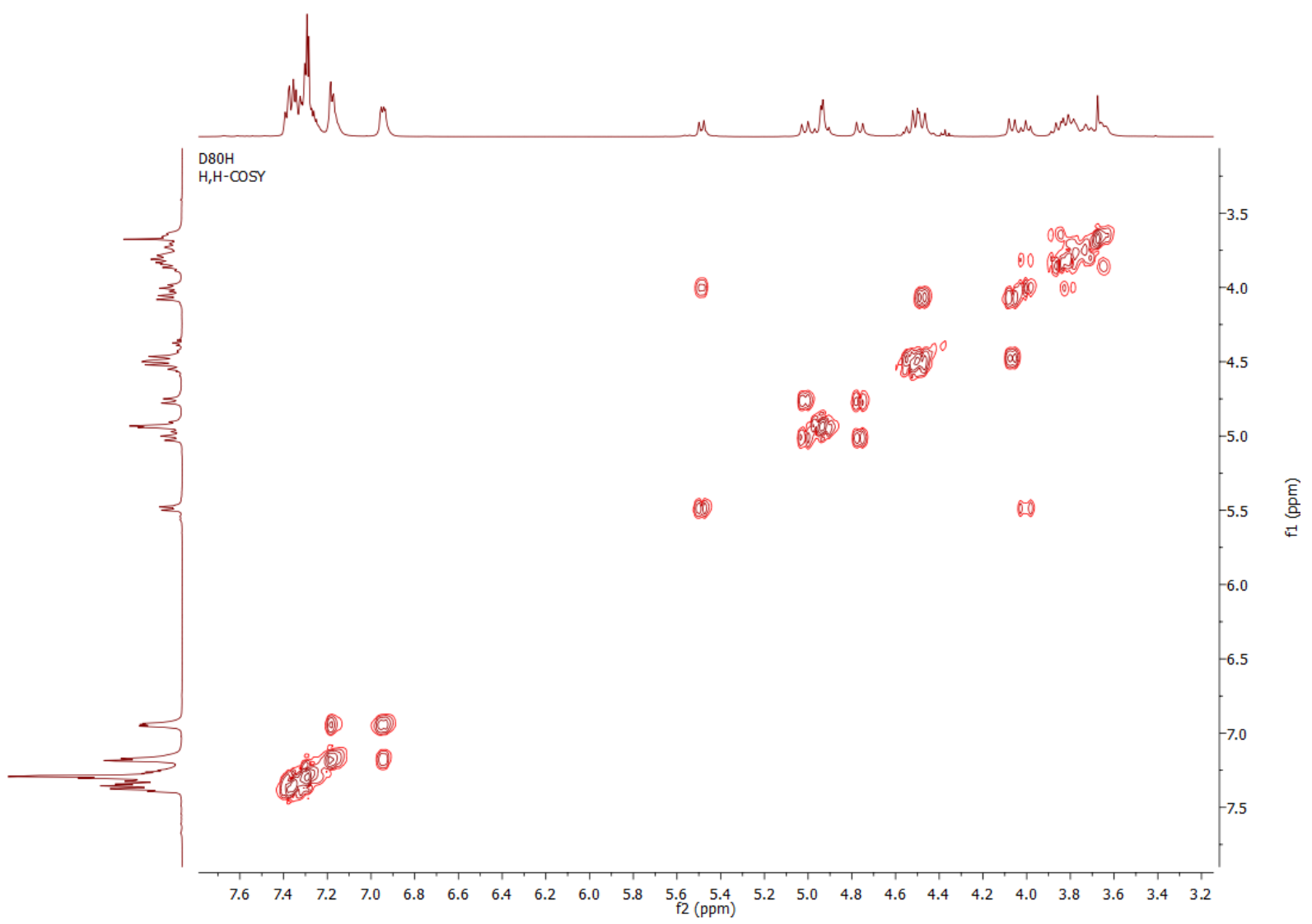

Figure S5. $\mathrm{H}, \mathrm{H}-\mathrm{COSY}$ NMR spectrum of $\mathbf{D}_{80 \mathrm{H}}$. 


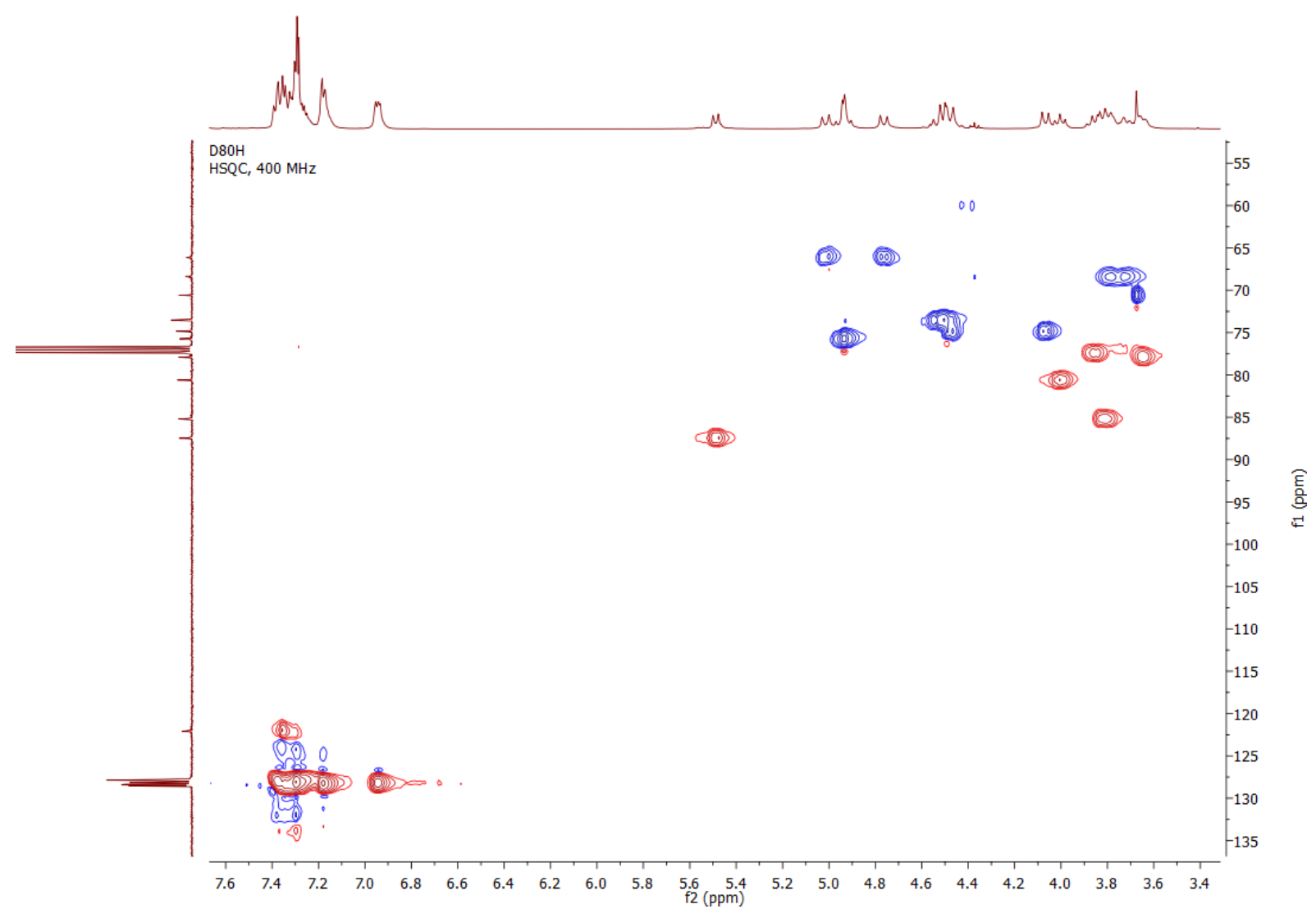

Figure S6. HSQC NMR spectrum of $\mathbf{D}_{\mathbf{8 0 H}}$.

a

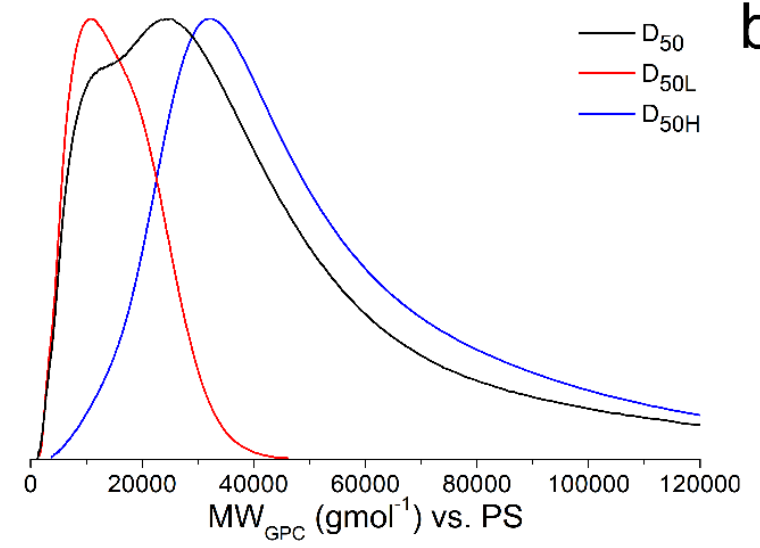

C

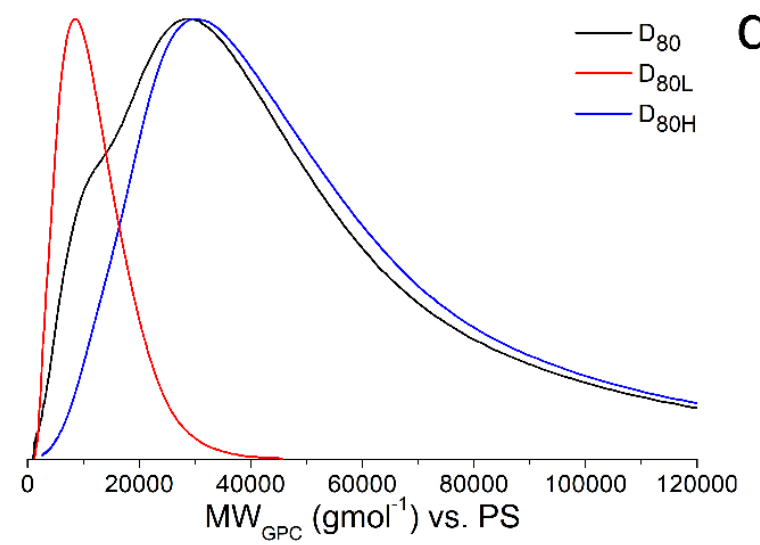

b

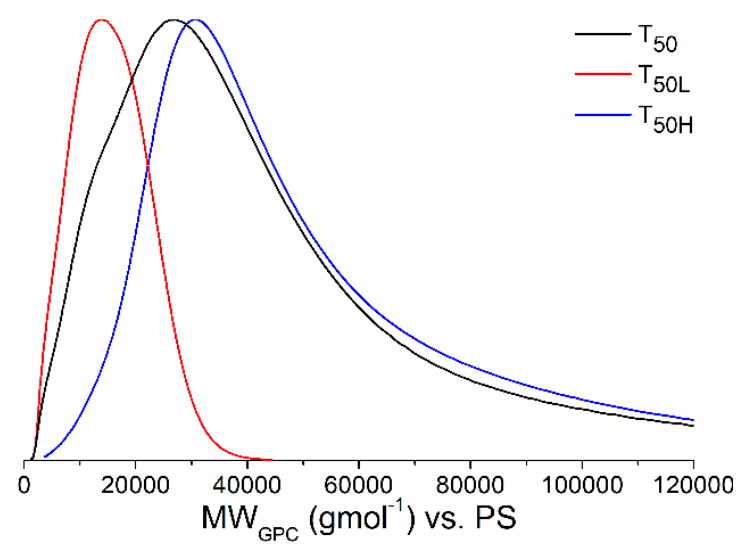

d

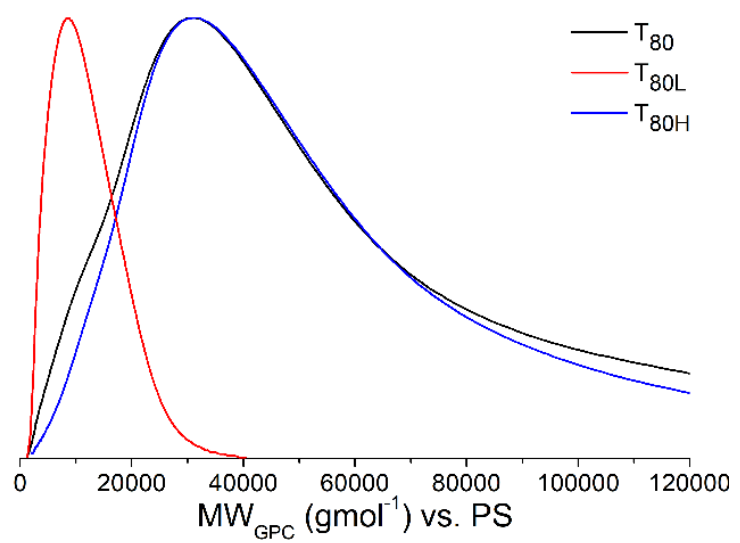

Figure S7. a. SEC curves of $D_{50}, D_{50 \mathrm{~L}}$ and $D_{50 \mathrm{H}}$. b. SEC curves of $T_{50}, T_{50 \mathrm{~L}}$ and $T_{50 \mathrm{H}}$. C. SEC curves of $D_{80}, D_{80 \mathrm{~L}}$ and $D_{80 \mathrm{H}}$. d. SEC curves of $T_{80}, T_{80 L}$ and $T_{80 H}$. 


\section{References}

(1) d'Arcy, R.; Tirelli, N. Mitsunobu Reaction: A Versatile Tool for PEG End Functionalization. Macromol. Rapid Commun. 2015, 36, 1829-1835.

(2) Su, L.; Zhao, Y.; Chen, G.; Jiang, M. Polymeric vesicles mimicking glycocalyx (PV-Gx) for studying carbohydrate-protein interactions in solution. Polym. Chem. 2012, 3, 1560.

(3) Pathigoolla, A.; Sureshan, K. M. Reverse-CD mimics with flexible linkages offer adaptable cavity sizes for guest encapsulation. Chem. Commun. 2014, 50, 317-319.

(4) Evans, M. E. Methyl 4,6-O-benzylidene- $\alpha$ - and - $\beta$-d-glucosides. Carbohydr. Res. 1972, 21, 473-475. 